Current Capabilities and

I : I: $:$ : $:$. Future Directions in

Computational Fluid Dynamics

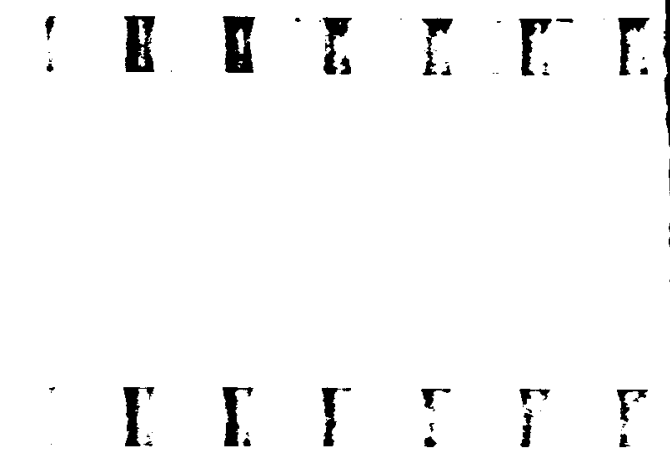

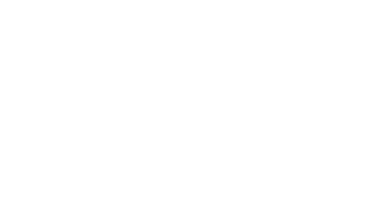

I

LILILI
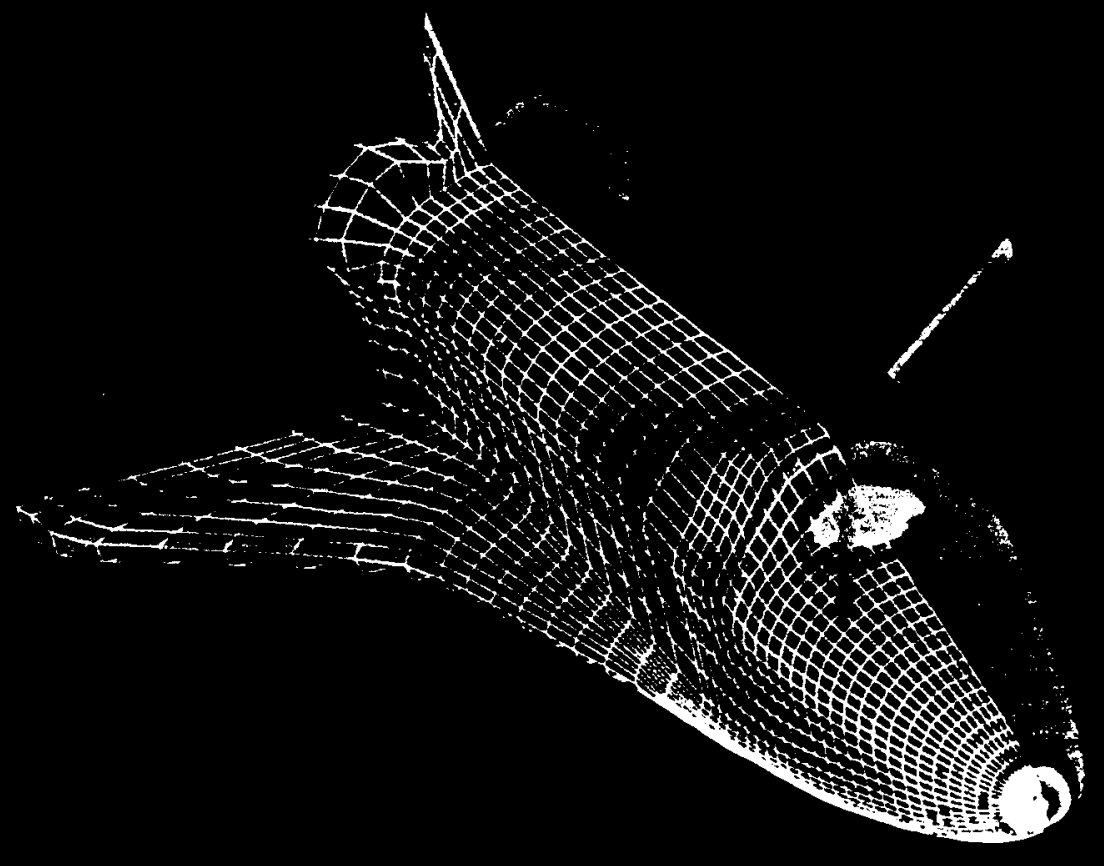

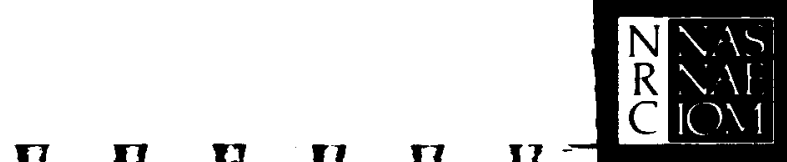

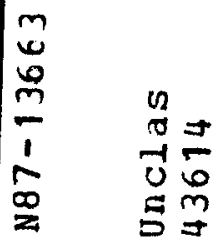

$\begin{array}{llllll}1 & 1 & 17 & \text { II } & \text { I } & \text { II }\end{array}$
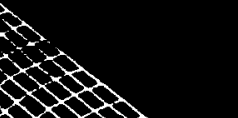

\title{
Todatáa
}




\section{CURRENT CAPABILITIES AND FUTURE DIRECTIONS \\ IN COMPUTATIONAL FLUID DYNAMICS}

Committee to Assess Current Capabilities and

Future Directions in Computational Fluid Dynamics Aeronautics and Space Engineering Board

Commission on Engineering and Technical Systems

National Research Council

NATIONAL ACADEMY PRESS

Washington, D.C. 1986

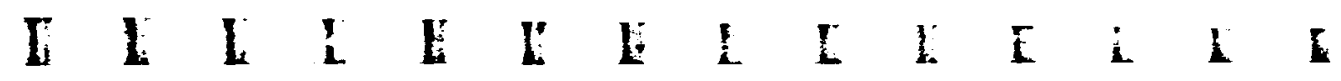


NOTICE: The project that is the subject of this report was approved by the Governing Board of the National Research Council, whose members are drawn from the councils of the National Academy of Sciences, the National Academy of Engineering, and the Institute of Medicine. The members of the committee responsible for the report were chosen for their special competences and with regard for appropriate balance.

This report has been reviewed by a group other than the authors according to procedures approved by a Report Review Committee consisting of members of the National Academy of Sciences, the National Academy of Engineering, and the Institute of Medicine.

The National Research Council was established by the National Academy of Sciences in 1916 to associate the broad community of science and technology with the Academy's purposes of furthering knowledge and of advising the federal government. The Council operates in accordance with general policies determined by the Academy under the authority of its congressional charter of 1863 , which establishes the Academy as a private, nonprofit, self-governing membership corporation. The Council has become the principal operating agency of both the National Academy of Sciences and the National Academy of Engineering in the conduct of their services to the government, the public, and the scientific and engineering communities. It is administered jointly by both Academies and the Institute of Medicine. The National Academy of Engineering and the Institute of Medicine were established in 1964 and 1970, respectively, under the charter of the National Academy of Sciences.

This study was supported by Contract NASW-4003 between the National Academy of Sciences and the National Aeronautics and Space Administration.

Copies available from:

Aeronautics and Space Engineering Board

National Research Council

2101 Constitution Avenue, N.W.

Washington, D.C. 20418

Printed in the United States of America

The cover shows the computed pressure field about the space shuttle Orbiter at a free stream Mach number of 1.4 and at zero angle-of-attack using the thin layer Navier-Stokes equations. The finite difference calculations were carried out on the CRAY-XMP using a segmented grid approach. (Numerical simulations by $Y$. Rizk and S. Ben-Schmuel)

\section{I $\quad$ I L}

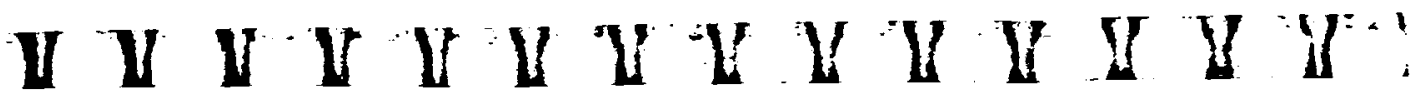




\section{COMMITTEE TO ASSESS CURRENT \\ CAPABILITIES AND FUTURE DIRECTIONS OF COMPUTATIONAL FLUID DYNAMICS}

RICHARD G. BRADLEY, Chairman, Director, Aerospace Technology, General Dynamics Corporation

DEAN R. CHAPMAN, Professor, Departments of Aeronautics/

Astronautics and Mechanical Engineering, Stanford University

WILLIAM H. HEISER, Vice President/Director, Aerojet Propulsion

Research Institute

JOHN L. LUMLEY, Professor, Sibley School of Mechanical and

Aerospace Engineering, Cornell University

ROBERT W. MacCORMACK, Professor, Department of Aeronautics and Astronautics, Stanford University

ROBERT E. MELNIK, Director of Fluid Mechanics, Grumman

Corporation

WILLIAM C. REYNOLDS, Professor, Department of Mechanical

Engineering, Stanford University

PAUL E. RUBBERT, Aerodynamics Technology Staff, Boeing Military Airplane Company

MICHAEL J. WERLE, Manager of Gas Dynamics and Thermophysics, United Technologies Research Center

DAVID L. WHITFIELD, Professor, Department of Aerospace

Engineering, Mississippi State University

\section{LIAISON REPRESENTATIVES}

JAY P. BORIS, Director, Laboratory for Computational Physics, Naval Research Laboratory

JOSEPH SHANG, Technical Manager, Computational Aerodynamics,

Air Force Wright Aeronautical Laboratories, Wright-Patterson Air Force Base

PETER M. SOCKOL, Assistant Chief, Computational Methods Branch, NASA Lewis Research Center

JERRY C. SOUTH, JR., Chief Scientist, NASA Langley Research

Center

JOSEPH STEGER, Senior Staff Scientist, NASA Ames Research Center

iii

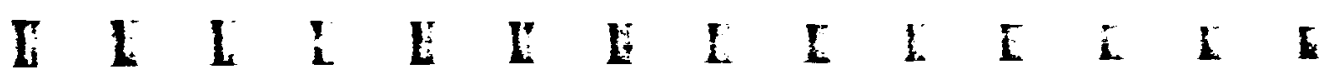


NASA REPRESENTATIVES

RANDOLPH A. GRAVES, JR., Office of Aeronautics and Space

Technology, NASA Headquarters

LEWIS L. PEACH, JR., Office of Aeronautics and Space Technology,

NASA Headquarters

STAFF

ROBERT H. KORKEGI, Study Director

JoANN CLAYTON, Assistant Study Director

ANNA L. FARRAR, Administrative Assistant

iv

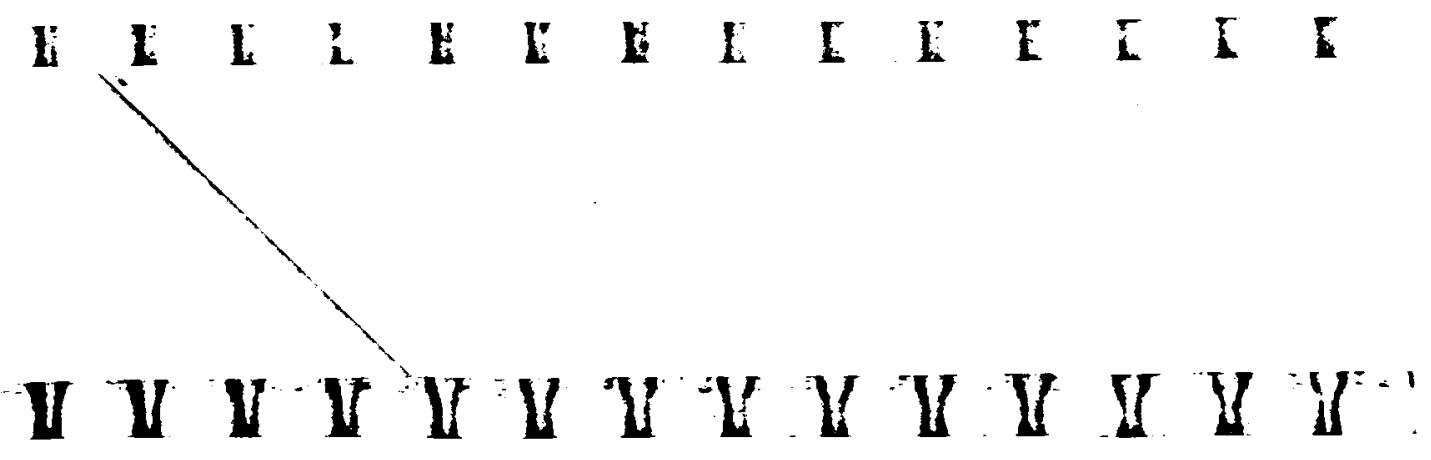




\section{AERONAUTICS AND SPACE ENGINEERING BOARD}

JOSEPH F. SHEA, Chairman, Senior Vice President, Engineering,

Raytheon Company

JAMES J. KRAMER, Vice Chairman, Manager, Advanced Technology

Programs, General Electric Company

MAX E. BLECK, Assistant to the President, Beech Aircraft

Corporation

BERNARD BUDIANSKY, Professor of Structural Mechanics, Harvard

University

W. BOWMAN CUTTER III, Partner, Coopers \& Lybrand

R. RICHARD HEPPE, President, Lockheed-California Company

RICHARD W. HESSELBACHER, Manager, Advanced Development and

Information Systems, General Electric Company

KENNETH F. HOLTBY, Senior Vice President, The Boeing Company

PETER W. LIKINS, President, Lehigh University

DONALD J. LLOYD-JONES, President and Chief Operating Officer,

Western Airlines

STePhen F. LUNDSTrom, Vice President and Program Director,

$\mathrm{M} \mathrm{C} \mathrm{C}$

ARTUR MAGER, Consultant, Los Angeles, California

STANLeY MARTIN, JR., Vice President-V-22 Engineering, Bell

Helicopter Textron

JOHN L. McLUCAS, Consultant, Alexandria, Virginia

IRWIN MENDELSON, President, Engineering Division, Pratt \&

Whitney Aircraft Group

JAN ROSKAM, Ackers Distinguished Professor of Aerospace

Engineering, University of Kansas

ROGER D. SCHAUFELE, Vice President, Engineering, Douglas

Aircraft Company

RICHARD S. SHEVELL, Professor, Department of Aeronautics and Astronautics, Stanford University

ROBERT E. SKELTON, Professor of Aeronautical Engineering, Purdue

University

ALTON D. SLAY, Slay Enterprises, McLean, Virginia

MORRIS A. STEINBERG, Consultant, Los Angeles, California

LAURENCE R. YOUNG, Professor of Aeronautics and Astronautics, Massachusetts Institute of Technology

$\mathbf{v}$

I I L L I L L L L L L L L L E 
EX OFFICIO MEMBERS

JOHN W. TOWNSEND, JR., Senior Vice President, Operations and

Planning, Fairchild Industries, Inc.

THOMAS M. DONAHUE, Department of Atmospheric and Ocean

Sciences, University of Michigan

STAFF

ROBERT H. KORKEGI, Director

JOANN C. CLAYTON, Senior Program Officer

BERNARD MAGGIN, Senior Program Officer

ANNA L. FARRAR, Administrative Assistant

LAURA D'SA, Administrative Secretary

REGINA D. MILLER, Senior Secretary 


\section{Contents}

1. SUMMARY OF SIGNIFICANT FINDINGS AND

RECOMMENDATIONS

Findings, 1

External Aerodynamics, 1

Hypersonics, 3

Propulsion, 3

Turbulence, 4

Recommendations, 5

2. INTRODUCTION

3. APPROACH

Survey of CFD Codes and Their Capabilities, 12

Survey of the National Level of Effort in CFD, 13

4. EXTERNAL AERODYNAMICS APPLICATIONS OF CFD

Overview, 17

CFD Capabilities in External Aerodynamics, 18

Capabilities for Steady Aerodynamic Flows, 20

Capabilities for Unsteady Aerodynamic Flows, 28

Findings, 31

Recommendations, 34

5. HYPERSONIC APPLICATIONS OF CFD

Overview, 35

Departures from Conventional Aircraft CFD, 37

CFD Capabilities in Hypersonics: Upper and Lower

Atmosphere, 39

Current Status of Hypersonic CFD, 40

Findings, 44

Recommendations, 45

6. PROPULSION APPLICATIONS OF CFD

vii

I I I I I I 
Overview, 47

Detailed Design of Components, 48

Development of Insight and Understanding, 49

Possibility for Revolutionary Change, 50

CFD Capabilities in Propulsion, 51

Stationary Systems, 52

Rotating Systems, 53

Combustors, 55

Findings, 57

Stationary Systems, 59

Rotating Systems, 59

Combustors, 60

Aerodynamic Coupling, 60

Recommendations, 61

7. TURBULENCE IN CFD

Overview, 63

Turbulence-Averaged Navier-Stokes Equations, 65

Capabilities of Turbulence Models and Simulation, 66

Local Algebraic Models, 67

Scale Evolution Models, 68

Transport Evolution Models, 70

Probability Density Function Models, 72

Spectral Interaction Models, 73

Turbulence Simulations, 74

Emerging Ideas, 76

Current Status of Turbulence Modeling, 77

Findings, 78

Recommendations, 79

APPENDIXES

A. Trends in Computer Hardware Affecting CFD, 83

B. CFD Capabilities Questionnaire, 86

C. Level of Effort Questionnaire, 92

D. List of Contributors to CFD Capabilities Questionnaire, 95

viii

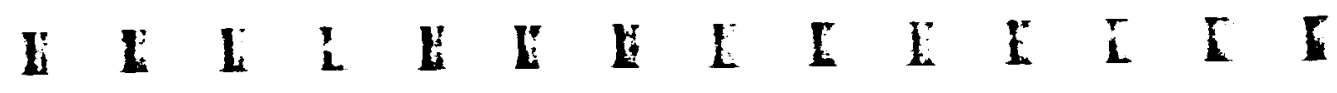

I Y V Y I Y Y Y Y U I I I I:" 


\section{TABLES AND FIGURES}

\section{CHAPTER 3}

Figure 1. The CFD Development Cycle 12

Figure 2. Breakdown of Resources Applied to.CFD 15

CHAPTER 4

Table 1. Status of CFD for External Aerodynamic Applications

\section{CHAPTER 5}

Table 2. Contrasts within Hypersonic CFD

Table 3. Status of CFD Capability-Hypersonic

Lower Atmosphere

42

Table 4. Status of CFD Capability-Hypersonic Upper

Atmosphere

CHAPTER 6

Table 5. Status of CFD for Propulsion Applications

\section{CHAPTER 7}

Table 6. Status of Turbulence Modeling 


\section{1 Summary of Significant Findings and Recommendations}

A summary of significant findings is given below, followed by specific recommendations for future directions of emphasis for computational fluid dynamics (CFD) development. The discussion is organized into three application areas-external aerodynamics, hypersonics, and propulsion-and followed by a turbulence modeling synopsis.

\section{FINDINGS}

\section{External Aerodynamics}

The aerospace industry has come to realize that CFD offers great potential as a design tool. In the commercial transport industry, where the aerodynamics encountered over much of the flight envelope represents attached flows and is well understood, CFD plays an integral part in the design process. In contrast, the design of high-performance military aircraft is usually dominated by more complex flow phenomena, not yet fully amenable to CFD analysis, with the result that CFD has had a lesser impact. However, this is changing rapidly.

CFD methods today can simulate flows about complex geometries with simple physics, or about simple geometries with more complex physics. They cannot do both. The most pressing need today is for algorithm technology that will lead to practical methods for solving the nonlinear flow equations-full-potential, Euler,

1

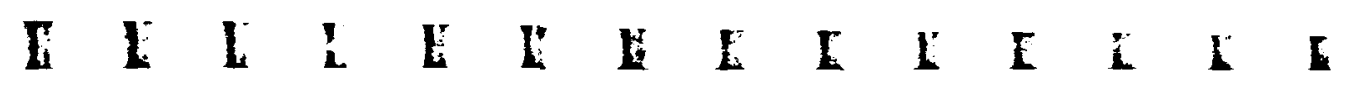


and the various forms of Navier-Stokes equations-about arbitrary and often complex geometries with reliable and predictable accuracy.

For steady aerodynamic flows, only the linear methods based on solutions to the Prandtl-Glauert equations have been developed to a mature state in that they provide capability for solving the flow about arbitrary and general configurations. Solution algorithms for the full-potential and Euler equations are reaching maturity for simple flows but remain low in the development cycle for flows of increasing complexity. Simulation capability for the 3dimensional (3-D) Navier-Stokes equations remains in a relatively primitive state although realization of the potential is providing impetus for increasing activity by the aerospace community.

CFD methods for unsteady aerodynamic flows have received far less attention than those for steady flows. Progress has been made in developing algorithms for the inviscid equations for relatively simple geometries. Some work has focused on coupling unsteady inviscid codes with steady boundary-layer and on unsteady 2-dimensional (2-D) Navier-Stokes codes. However, the viscous unsteady CFD technology is in its infancy.

Although today's algorithms for the Euler and Navier-Stokes equations are more accurate and robust than those of yesterday, they are still unsatisfactory in many respects. Major improvements are needed in spatial accuracy and solution adaptability, convergence reliability, and convergence rates. Worthwhile codes have been produced, but further improvements in algorithm technology could multiply their usefulness many times over. Turbulence modeling will become a major issue for Navier-Stokes solvers, but with today's algorithms the various errors associated with numerics are probably a larger issue in need of resolution. When those sources of error are resolved, then turbulence modeling will become the limiting factor.

There are other areas that should not be neglected. Needs and opportunities exist throughout the spectrum of CFD; codes solving linear flows, the boundary-layer equations, full-potential equations, and Euler and Navier-Stokes equations all will have a role to play in the aircraft industry for the foreseeable future.

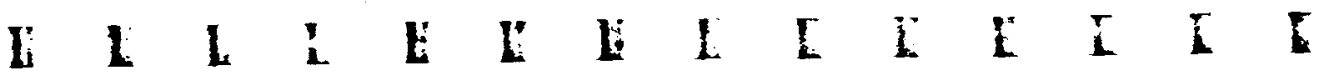

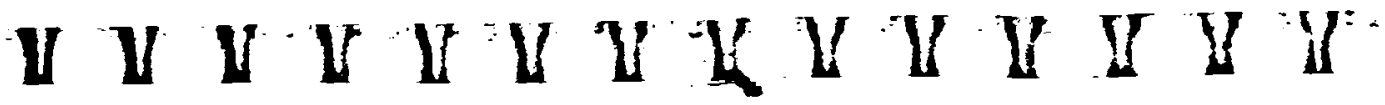




\section{Hypersonics}

Hypersonic $\overline{C F} \bar{D}$ spans a wide range in complexity dependent mainly on flight altitude. In the lower atmosphere, air chemistry is in thermodynamic equilibrium, the flow is transitional and turbulent, and the corresponding CFD codes are only a modest extension of those for conventional aircraft. In the upper atmosphere, nonequilibrium air chemistry prevails and the flow is mainly laminar. The corresponding CFD codes are much more complex and in a more primitive state of development due to numerous additional molecular physics processes that must be considered.

The simulation of hypersonic flow fields undergoing nonequilibrium chemistry requires that vehicle scale be simulated as well as flight velocity and altitude. Because this is not possible in existing ground-based experimental facilities, the applications and the importance of CFD to hypersonic vehicle design is much greater than for conventional aircraft design. For flight in the upper atmosphere, CFD is expected to be the principal source of flow simulation information for vehicle design, and flight test is necessary for accurate validation of the computational codes.

\section{Propulsion}

Computational fluid dynamics is used extensively throughout the propulsion community because of a lack of alternatives for achieving insight into the basic controlling mechanisms of the complicated, highly coupled interacting aspects of propulsion fluid dynamics. It has proven a valuable design analysis tool in a wide variety of situations, often more than expected. Use of CFD is growing rapidly largely because of its ability to provide detailed diagnostic data and to address more complex flows. One of the principal challenges now being faced is to provide a way for this powerful but costly capability to contribute directly to the formal design of complicated, strongly coupled propulsion system components.

In general, propulsion CFD is in a relatively mature state for 2-D potential flows with capability dropping off systematically for increased levels of complexity. This can be better summarized in tern:s of the three major elements of propulsion devices: stationary systems, rotating systems, and combustors. For stationary systems, CFD technology is in a relatively mature state for 2-D

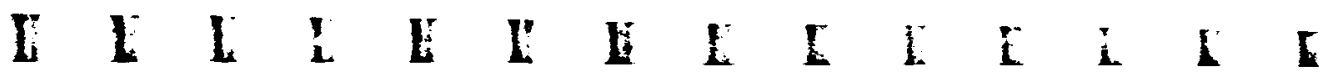


configurations, with a rapid drop in capability for 3-D or unsteady flows. For rotating systems, capability naturally lags behind the stationary counterpart at all levels-most seriously in the area of viscous flow modeling. Combustors, inherently more complicated, show the lowest level of CFD development.

The strong aerodynamic coupling of the major components of propulsion systems is another important issue. Examples of this include the influence of inlet distortion on engine performance, stability, noise, and durability of the fan; and the impact of burner exit temperature profiles on turbine life and stagnation (nonrecoverable) stall. With the advent of larger capacity parallel processing computers, it may soon be possible to model interacting components.

The area of hypersonic propulsion systems should be highlighted because of interest in advanced engines for hypersonic and transatmospheric vehicles. The flow conditions in these machines are so demanding that in many cases they cannot be reproduced in existing facilities. Even though existing codes have inadequacies in turbulence and chemistry modeling, they offer the only available capability and will carry an unusually large share of the design burden.

There is a need for benchmark experiments to provide detailed data bases against which codes can be calibrated to assist and guide the development, especially with respect to turbulence modeling and hypersonic propulsion systems. Diagnostic experimental studies are needed to provide detailed data to allow identification of the dominant physics, thus providing critically needed insight for establishing and validating appropriate levels of analytical modeling.

\section{Turbulence}

In complex engineering flows where strong viscous effects dominate, turbulence modeling, including modeling of the laminarturbulent transition, is becoming a pacing technology. Present turbulence models are adequate only for use in relatively simple flows, and do poorly in flows with strong 3-dimensionality, massive separation, large-scale unsteadiness, strong density gradients, strong rotation, and chemical reaction. Large eddy simulations (LES) have not yet been developed for boundary conditions, geometries, and Mach numbers of practical interest. It is important

\section{L $\quad$ I L L I}


to develop LES for use in critical portions of complex turbulent flows, but it will be a very long time, if ever, before LES is applied to a full aircraft flow. Full turbulence simulations (FTS) (all scales) will be limited to simple flows at relatively low Reynolds numbers. While useful in fundamental studies of turbulence, the FTS limitations are such that they are not likely to find much use in engineering applications in the foreseeable future. Hence, some form of turbulence modeling, and modeling of transition, is expected to remain an essential element for engineering CFD.

It is in the most complicated flows, where intuition fails and basic experiments are missing, that the turbulence models perform most poorly. With the advent of effective methods for solving the flow equations, the inadequacies of turbulence modeling may become the pacing item in the future application of CFD to practical vehicle design.

The other items pacing the development of engineering CFD are more amenable to rapid advance, as has already been shown in the case of grid generation, algorithms, and hardware. Solving the turbulence problem is a tough, long-standing issue of growing importance that will require sustained attention by many interacting, highly competent groups.

The current level of effort in the development of better turbulence models is limited. The advent of FTS as a tool for gathering data about turbulent flows, advances in the methodology of rational structuring of models, and new experimental techniques offers promise for more rapid advances in turbulence modeling.

\section{RECOMMENDATIONS}

The ultimate goal for CFD is the development of a fully mature capability that is user-friendly, cost-effective, and fully verified by detailed experimental comparison. The committee feels that order-of-magnitude improvements are possible and should be aggressively sought. In this context the following recommendations are made as a guide for future directions in CFD.

\section{Algorithm Technology}

Stronger emphasis is needed on the development of more advanced algorithm technology, particularly for the Euler equations and the various forms of the turbulence-averaged Navier-Stokes equations. Four objectives are recommended.

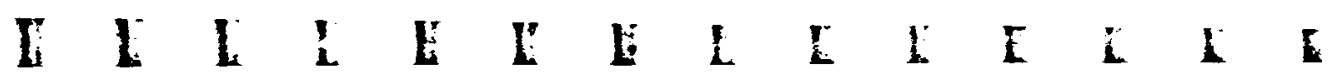


1. Advances to enable computation of flows about complex geometries in a manner that is practical and accurate. Such technology must include means for resolving phenomena that occur at the differing length scales found in nonlinear flows such as thin boundary layers, shock waves, free shear layers, exhaust plumes, and regions of separated flow. Focus should be given to developing smart difference schemes, including adaptive grids, to achieve the required balance.

2. Major improvements in spatial accuracy, convergence reliability, and convergence rates. Reduction of numerical diffusion, artificial dissipation, and smoothing procedures is needed. Most large codes are saddled with "knobs" that are empirically set to obtain the best balance between accuracy and convergence. Major advances in these areas are mandatory before CFD can be relied upon widely for design applications throughout the aerospace community.

3. Verification of flow models and algorithms. General acceptance of CFD as an established design tool can only be predicated on confidence gained from comparison with detailed experimental data to validate the numerical model used to simulate the flow physics.

4. Analytical and computational modeling of aeroelastic loadings on aircraft lifting components, blades, and propellers. CFD methods should be extended to model structural aeroelastic coupling.

\section{Turbulence Simulation and Modeling}

For developments in turbulence modeling to keep pace with the other items limiting the effectiveness of engineering CFD, the level of effort in turbulence modeling and simulation should be increased in a coordinated way. New opportunities for study and research in turbulence modeling and simulation and coordination among experts are needed. Three objectives are recommended.

1. Turbulence/transition modeling for use in the turbulenceaveraged Navier-Stokes computations. While significant and rapid progress is being made in most other related areas, little work is being focused on this item. Work is needed in development of new turbulence models both time-averaged and time-resolved, focusing on freestream disturbance effects (e.g., unsteadiness, turbulence, and vorticity), transition prediction, and compressible flows.

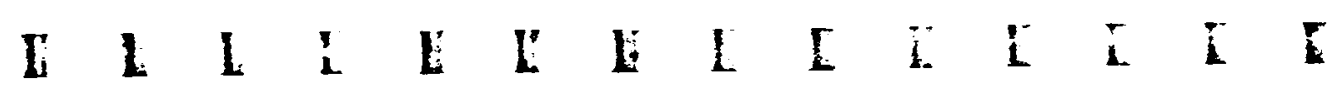

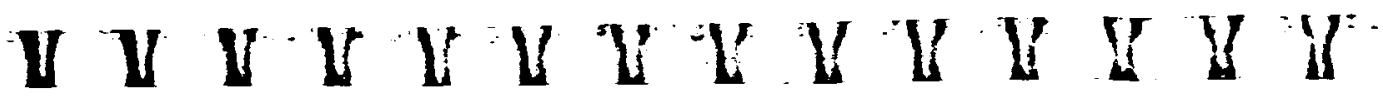


2. Continued emphasis on large eddy and full turbulence simulations. While the simulations may never be developed to a level necessary for routine design application, they may be able to provide a critical data base for fundamental understanding and a framework for developing practical turbulence models for use in solving the turbulence-averaged Navier-Stokes equations. In addition, these simulations have the potential to solve certain very difficult portions of practical flows.

3. Generation of benchmark and diagnostic experimental data bases at a time-resolved level. Benchmark experimentation combined with the LES/FTS numerical experimentation will aid in developing and verifying new turbulence and transition models; this is especially needed in the propulsion area.

\section{Hypersonic Directions}

The emerging importance of CFD as a tool for hypersonic system design and analysis has opened new areas of uncertainty, especially in the upper atmosphere where nonequilibrium chemistry and transition play dominant roles. Specific emphasis is recommended in five areas.

1. Continuum equations of motion. Some inaccuracies in the conventional Navier-Stokes equations for hypersonic flow conditions need to be mended and new physics added to the continuum equations of motion if they are to integrate accurately through shock waves in the upper atmosphere.

2. Molecular physics data base. Extensive physical data required as inputs for hypersonic CFD codes are needed on chemical reaction rates, excitation rates, gas-surface reactions, and transport properties at high temperature.

3. Transition and turbulence. These problem areas can have a major impact on aerodynamic heating and payload weight.

4. Numerical methods. Algorithms with improved computational efficiency for both continuum and particulate flow simulations are needed to reduce the computer time required to simulate flows with important chemical nonequilibrium effects.

5. Flight tests. Appropriately instrumented flight tests are necessary to validate computational codes for hypersonic flight in the upper atmosphere due to the inability of ground-based experimental facilities to simulate properly chemical nonequilibrium.

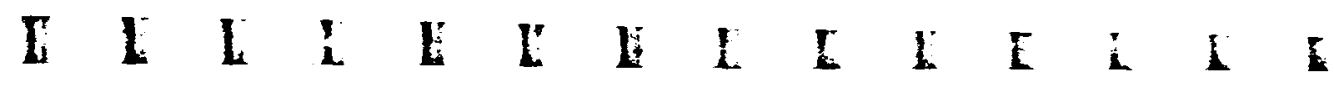




\section{Introduction}

In recent years national attention has become focused on the development of a national computational fluid dynamics (CFD) capability. ${ }^{*}$ Significant advances in numerical algorithms, processing speed, and storage capacity of new generations of computers prompted the present study to assess the current capability and future directions for CFD.

The study was requested and supported by the Office of Aeronautics and Space Technology of the National Aeronautics and Space Administration (NASA) and was undertaken by an ad hoc committee of the National Research Council's Aeronautics and Space Engineering Board. The committee's membership represented a broad base of expertise in the development and application of CFD capabilities in internal flow and external flow across the Mach number range from subsonic to hypersonic flow as well as expertise in turbulence modeling.

The specific charge to the committee follows:

Computational fluid dynamics (CFD) is becoming an increasingly powerful tool in the aerodynamic design of aerospace systems as a result of improvements in numerical algorithms as well as in the processing speed and

* CFD provides the numerical solution of partial differential equations or integral equations covering fluid flow. For the purpose of this report, the nomenclature of computational fluid dynamics (CFD) is used to broadly cover the computation of fluid flows about or within bodies regardless of solution method and must be understood to encompass a range of related areas such as combustion, rarified gas dynamics, computational aerodynamics, and computational aerothermodynamics.

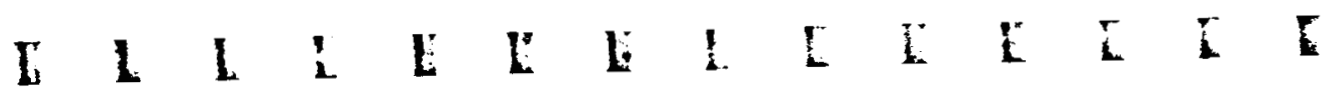


storage capacity of new generations of computers. As the next generation of supercomputers becomes available much current CFD work may be expanded to address more complex configurations, geometries, flight regimes, flow fields, and applications, while some of the existing work will become components of more complex systems of solutions to problems of modeling, code generation, flow field solvers, and flow visualization. Thus, adaptations and extensions of current work are expected to provide part of the foundation upon which the next level of CFD development will advance.

These advances in CFD as an aerospace-system design tool are proceeding at such a rapid pace that their full capability is not widely known by the aerospace community. This study will address the current state of the art in computational codes and supporting software for aerodynamic and aerothermodynamic applications for:

- external and internal flows,

- all speed regimes, and

- basic and applied research and development.

The committee will identify sources for unclassified CFD codes from government research laboratories, industry, and academia and will develop the fundamental assumptions and technical approach necessary to categorize codes by:

- application (configuration/geometry), - level of approximation, and flow - complexity.

Limitations or restrictions of specific codes or categories of codes should be identified, and software architecture should be specified as it relates to compatibility of various computer architectures.

The committee will assess the current status of CFD, identify any areas requiring a change in emphasis, and make recommendations regarding future directions and needs.

The approach used by the committee in addressing its charge is discussed in Chapter 3.

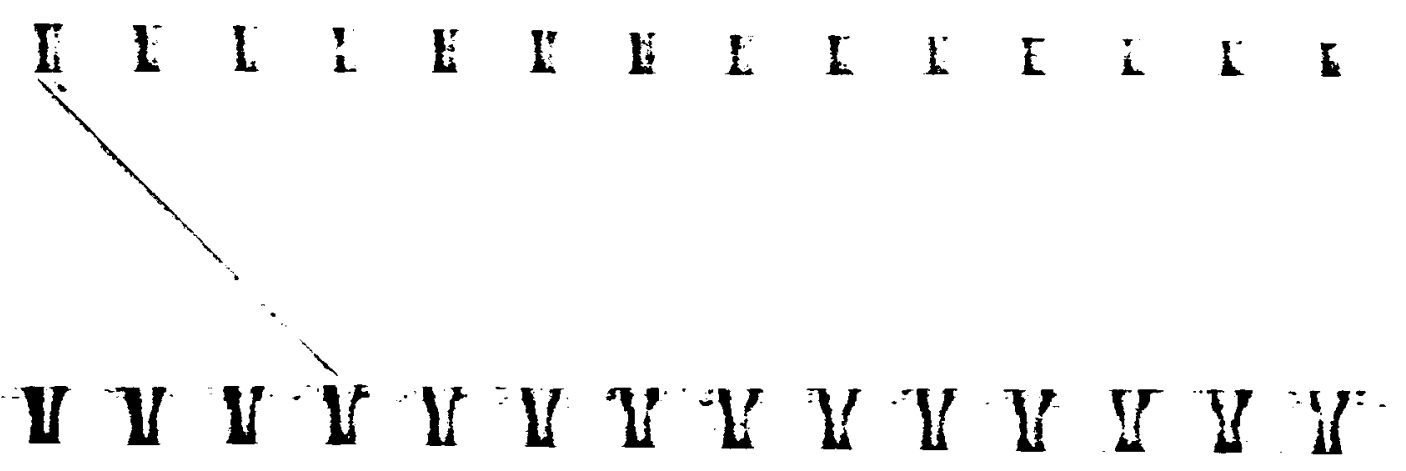


While the committee's charter did not include an evaluation of current or future developments in computer hardware, the close relationship between CFD capability and hardware is recognized. Appendix A contains some comments on expected trends in supercomputer evolution as a supplement to the study.

Members of the committee wish to express their gratitude to the individuals who served as liaison representatives for their organizations and who worked with the committee in developing the report, often making important contributions requiring considerable time and expertise. We also would like to thank their organizations for making their services available to us. These individuals are identified on page iii, along with the committee membership. We would especially like to acknowledge the valuable assistance of I. C. Bhateley of General Dynamics, who provided a LOTUS data tase and statistical analysis of the CFD Capability Survey responses. Last, thanks are due to those who contributed to the Level of Effort Survey and to those who responded to the CFD Capability Survey with specific code information. A list of contributors appears in Appendix D.

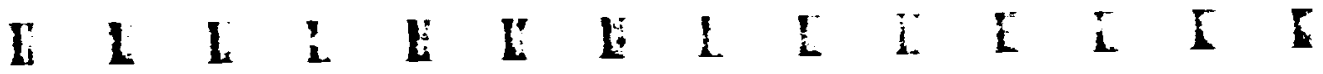




\section{3 \\ Approach}

The committee held three 2-day meetings as follows: July 2-3, 1985, at NASA Ames Research Center, Moffett Field, California; August 27-28, 1985, at the University of Texas, Arlington, Texas; and February 17-18, 1986, at the Fort Worth Division of General Dynamics, Fort Worth, Texas. In addition, a caucus of subgroup leaders was held on January 9, 1986, in conjunction with the American Institute of Aeronautics and Astronautics Aerospace Sciences Meeting in Reno, Nevada.

To assure equivalent coverage of important CFD application areas, the committee was divided into four subgroups in the following areas: external aerodynamics, hypersonics, propulsion, and turbulence. Chapters bearing these headings represent the consensus of the committee.

To provide a quantitative measure of current capabilities in CFD, the committee adopted a numerical scale based on position in the CFD development cycle. This cycle is depicted graphically in Figure 1. This grading system is used within subsequent chapters of this report to arrive at overall approximate evaluations of CFD capabilities. Thus, in the chapters on external aerodynamics, hypersonics, propulsion, and turbulence a grade of 1 represents Phase I of the development cycle where the basic elements of the enabling technology are formulated and a grade of 5 signifies a fully mature capability for dependable, cost-effective design applications, i.e., Phase $\mathrm{V}$ in the development cycle. Grades 2, 3, and 4, then, correspond to Phases II, III, and IV, respectively.

Two surveys were conducted during the course of the study. One solicited an evaluation of current CFD capability from a

11

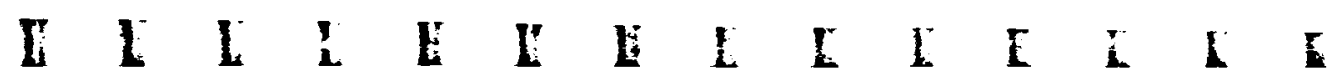

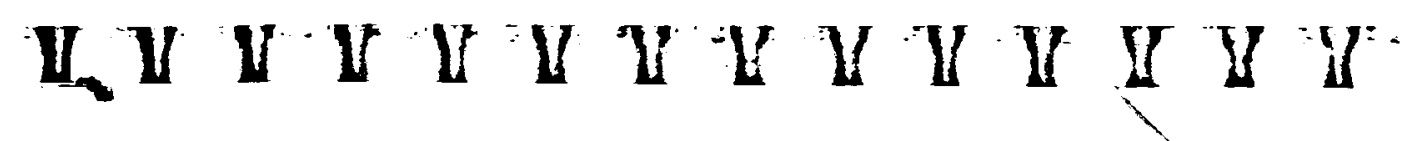




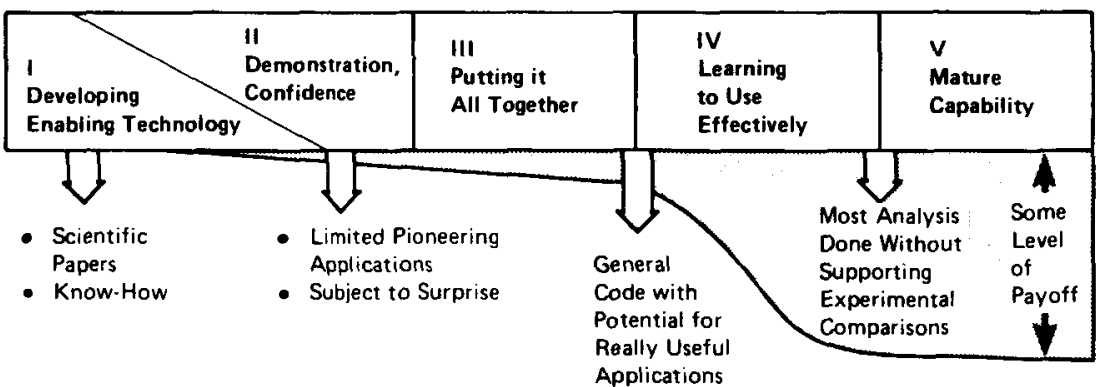

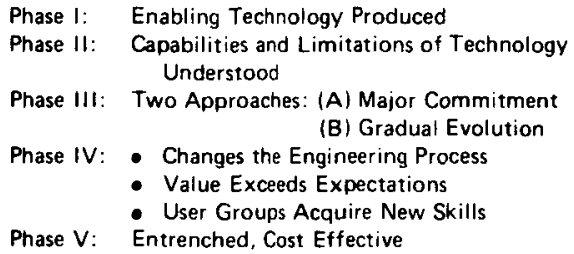

FIGURE 1 The CFD Development Cycle.

wide selection of known workers in the field. The other solicited assessments of the current level of effort for fundamental work in CFD and turbulence from government agencies known to be sponsoring such work. The surveys are described below.

\section{SURVEY OF CFD CODES AND THEIR CAPABILITIES}

To provide a grassroots assessment of U.S. capabilities in CFD a nationwide survey was conducted. The four-page survey format, reproduced in Appendix B, solicited information on the category of code application, governing equations solved, usability of the code, believability of the resultant solutions, and the code's place on the CFD Development Cycle as described above. While it is recognized that significant CFD work is being accomplished outside the United States, the survey was restricted to work within the nation in order to place some reasonable bounds on the scope of the study.

The purpose of the survey was twofold: (1) to provide the sponsoring agency, NASA, with a data base of current capability in the CFD field for use in planning activities, and (2) to provide the committee with a source of statistical information. From the

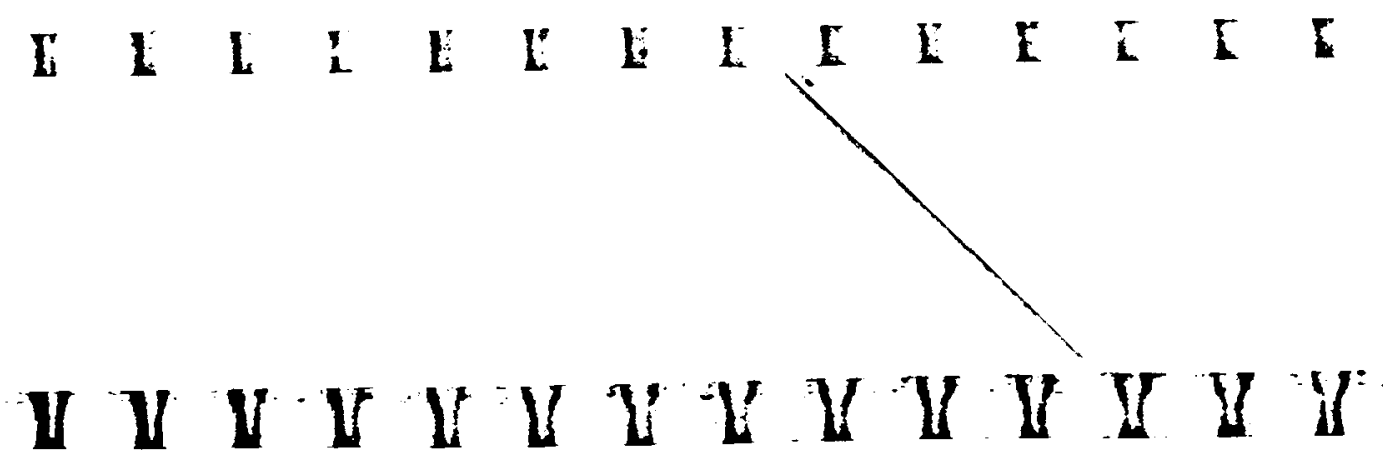


outset it was acknowledged that responses to many of the survey questions would be subjective. Nevertheless, the responses provided a valuable stimulant to the committee's deliberations.

Survey forms were mailed to 244 individuals known to be active in the development and application of CFD methods, and information was received regarding 259 codes from 142 sources. Of the responses, 139 represented seven government agencies, 84 represented 21 industrial or specialty companies, and 19 represented 14 universities. All responses that were received in the requested format were tabulated into a LOTUS spread-sheet format by I. C. Bhateley, who then provided the committee with summary data.

All of the survey data were supplied to each committee member along with the summary in spread-sheet form. The data were used by the committee throughout its deliberations and contributed to the development of the quantitative evaluations of the Status of CFD and Turbulence Modeling provided in tables in the report. It was the opinion of the committee that publication of the survey responses in detail was not justified for several reasons.

1. The data are too massive to permit practical publication.

2. Many of the inputs were repetitive in that the codes were the same or were variations of the same basic code.

3. In some cases the information furnished was not sufficiently well defined to substantiate the claimed capability.

Nevertheless, the survey results were extremely valuable when weighted with the individual committee member's knowledge and judgment. In addition, the entire survey has been supplied to NASA for possible compilation into a list of codes available within the United States. The large number of responses gives evidence of the high degree of interest in the field.

\section{SURVEY OF THE NATIONAL LEVEL OF EFFORT IN CFD}

The survey of the level of effort being devoted to developing CFD methods and turbulence modeling was limited to a selected list of government agencies that were known to be sponsoring research in CFD. The intent of the survey was not to determine the total resources directed toward CFD work but, rather, to obtain a representative sample of research activity from sponsoring government agencies. The committee believed that such data from

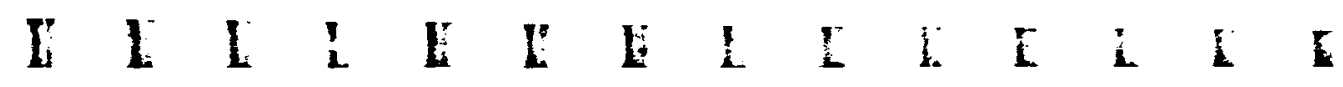


some of the major sponsors of CFD research would provide an indication of the principal focus in the field.

The form developed for this survey is reproduced in Appendix $\mathrm{C}$ along with the specific instructions that accompanied the form. Responses were received from the Air Force Office of Scientific Research, Air Force Wright Aeronautical Laboratories, Office of Naval Research, Naval Research Laboratory, NASA, and the Army Research Office. The survey was not sent to industry or universities although the responses include some work contracted to industry and universities. The responses received represent an effort of about 531 equivalent person years, and are considered to be a representative sample of effort in the field.

For the purpose of analysis, the data were generalized into five specific areas of activity. These areas consist of applications, fundamental development, experimental verification, physical modeling, and turbulence simulation.

A summary of the results of the survey appears in Figure 2. It shows the greatest effort, 34 percent, is directed toward applications of existing CFD codes. Fundamental development and experimental verification occupy a respectable amount of the total resources. It can be seen that a relatively small effort, 11 percent, is being put forth in the area of physical modeling and only 5 percent in the area of turbulence simulation. The physical modeling category is further broken down to illustrate that about 4 percent of the total CFD effort is directed toward turbulence model development.

In interpreting the survey results, it should be noted that all categories of effort represent important building blocks and are essential for developing a fully verified national CFD capability. Current needs are seen in fundamental algorithm development to provide solution and grid generation capability. Efficient algorithms are required for both viscous and inviscid flows over complex geometries. Detailed experimental verification is key for providing the level of confidence necessary for design applications. All of these are discussed further in the following chapter.

As basic algorithm development continues to mature, it appears inevitable that greater importance will be placed on accurate turbulence modeling and simulation, especially for flows that are dominated by viscous phenomena. However, the level of effort in these areas, as shown by the survey samples, is low. Turbulence

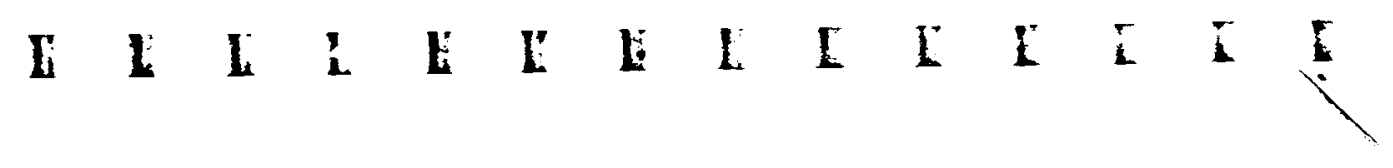




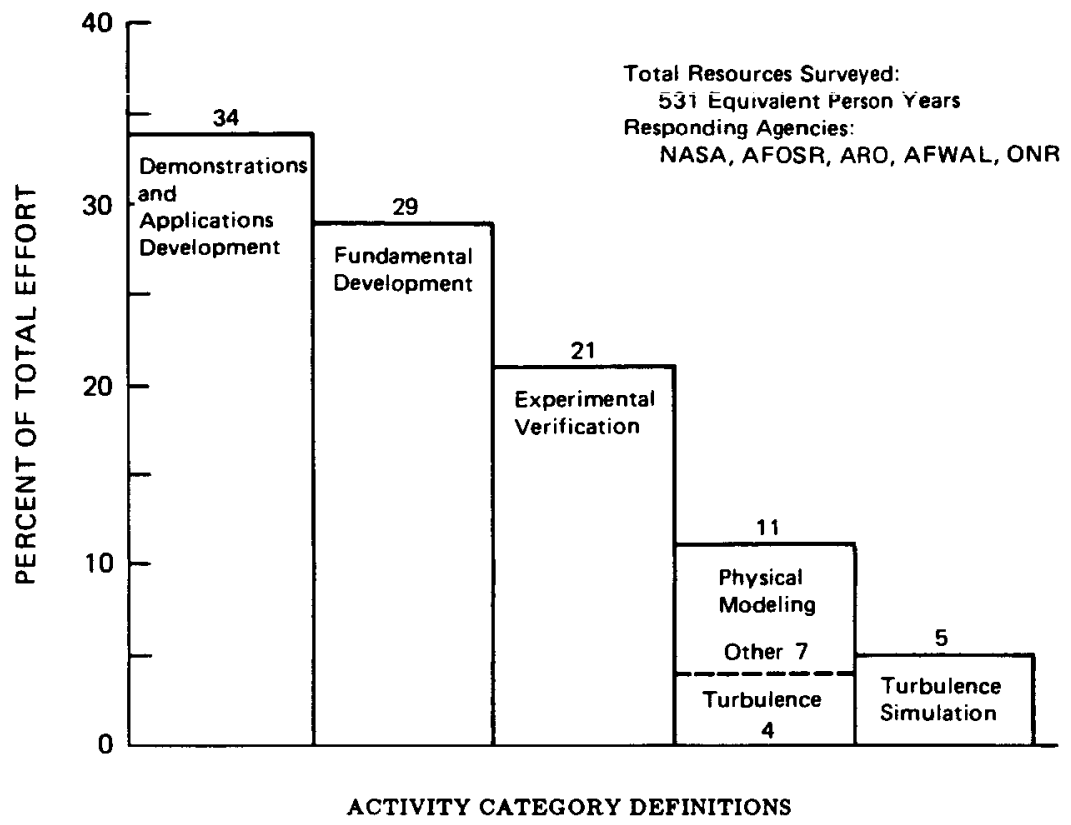

\begin{abstract}
DEMONSTRATIONS AND
- Code execution for evaluation and verification of codes APPLICATIONS DEVELOPMENT and models for specific application to phyaical flows. Includes teating of basic concepte and simulation methodology, providing predictive analysis capability, and initial support in disseminating codes to the user community.
\end{abstract}

FUNDAMENTAL DEVELOPMENT - Development of algorithms, grid generators, diagnostics or display software for potential flow, Euler, and Navier-Stoken solvers. Includes numerical analysis, stability and convergence analysis and research codes applying new techniques.

EXPERIMENTAL VERIFICATION - Baseline canonical or complex experiments designed to guide, calibrate, and/or evaluate modeis and codes. Includes both laboratory experiments snd computer experiments for eddy simulation and "benchmark" exeriments to gather detailed surface and flow field quantities.

PHYSICAL MODELING

- Physical model development including generalization of existing models, new ideas for model formulation, distillation of simple models from complex ones, and fabrication of epecial models for simple cases. Includes modeling of turbulence, heat transfer, chemical reactions, tranoition, mixing, flame-holding, and flow at ability.

TURBULENT SIMULATION

- Development of algorithms, grid generators, diagnostics or display software for Large Eddy Simulation/Full Turbulence Simulation for time-dependent three-dimensional eddy imulation.

FIGURE 2 Breakdown of Resources Applied to CFD.

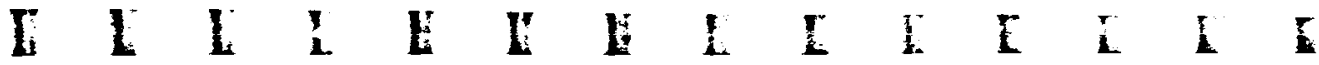


simulation work is almost exclusively directed toward incompressible flows. Compressible turbulence modeling and simulation is required for most flows of interest. Of perhaps even more concern is the small number of workers known to be skilled in turbulence simulation. This subject is discussed in greater detail in the chapter on turbulence modeling.

In summary, the committee found the information gleaned from both surveys useful and stimulating in its deliberations and greatly appreciates the input it received from the U.S. CFD community.

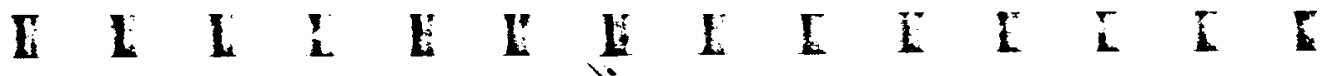




\section{4 \\ External Aerodynamics \\ Applications of CFD}

\section{OVERVIEW}

External aerodynamics encompasses a host of opportunities and needs for the application of CFD. Geometrical shapes of interest range from simple 2-D airfoils to complete 3-D airplanes involving not only the major components of wing, body, and tail but also the presence of propulsion systems, fillets and fairings, flap track fairings, vortex generators, slotted leading and trailing edge flaps, antennas, cavities, bleed holes and ports, and surface excrescences. The dominant fluid physics can range from simple incompressible inviscid flow to the most complex, unsteady, turbulent flow. The insights sought from CFD can range from prediction of steady-state force coefficients to detailed revelation of complex fluid dynamic phenomena.

Airplanes must perform and be demonstrated throughout a flight envelope. Within that envelope the flow about a typical airplane component can change from simple, low-speed attached flow to the most complex, separated, and unsteady behavior. Current CFD methods have only demonstrated an ability to simulate flows about complex geometries with simple physics or about simple geometries with more complex physics. In general, they cannot simulate flows about complex geometries with complex physics. Hence, one finds both CFD and experimental simulation facilities being used, in complementary ways, in the airplane design and verification process, with each being used for those things that it does best.

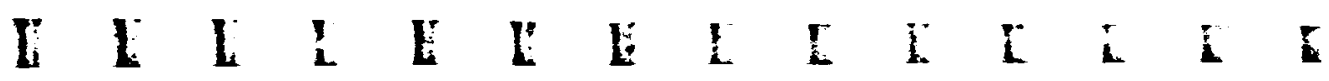


In the world of commercial transport aircraft development, the aerodynamics encountered over a large portion of the flight envelope are smooth, attached, and well understood. Such aerodynamics have become increasingly amenable to CFD in recent years with the result that it has come to play an integral role in the design process for modern transport aircraft.

In contrast, the design of high-performance military aircraft is usually dominated by more complex flow phenomena over much of the flight envelope. These flows have not been amenable to CFD in the past, with the result that CFD has had a lesser impact on high-performance military aircraft. However, that situation is rapidly changing. Computer developments and algorithm technology have reached the point where CFD can begin to contribute to the understanding and prediction of more complex flows, and those companies engaged in the military airplane business are becoming increasingly aggressive in the development and pioneering applications of CFD.

The high level of interest in hypersonic and transatmospheric vehicles within the United States is propelling the development of CFD for those applications. Hypersonic vehicle geometries are frequently less complex, allowing surface-fitted grid methodology to be effective in computational analysis. The flow is usually strongly hyperbolic in character, which means that in the case of equilibrium chemistry, iterative solution processes may converge in relatively few iterations. There is a dearth of experimental facilities capable of reproducing certain hypersonic flows because of severe physical requirements-full pressure and enthalpy simulation. Thus, CFD methods for hypersonic flows, including the effects of finite rate fluid chemistry are being aggressively developed. Chapter 5 of this report addresses in greater depth activities within the hypersonic area.

\section{CFD CAPABILITIES IN EXTERNAL AERODYNAMICS}

A CFD capability is comprised of many factors. No single code can reproduce all the physical phenomena. It is typical to find that a given code, for a given application, can simulate certain features well and other features not so well. One cannot in general make a categorical statement concerning whether a code can in

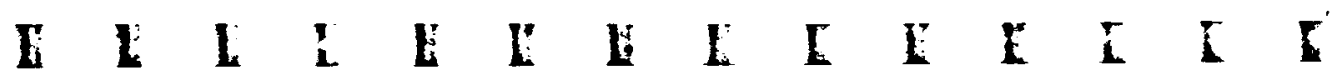


fact simulate a given flow. It depends entirely upon what one seeks to learn about a flow.

Perhaps the easiest feature to compute for many flows and codes is the overall lift. Lift is the result of an integration of forces exerted on a configuration by the fluid. The affect of viscosity for attached flows is usually small and small local errors in the computation have only minor effects on computed lift. Moments are usually somewhat more difficult to calculate accurately because they can be more sensitive to small errors in local pressure.

Surface pressure distributions are the most common feature typically displayed as the output of a CFD code, and it is common to make an overall judgment of a code based on the perceived accuracy of calculated surface pressures. However, such an assessment may fall short of revealing a code's true strengths and weaknesses. Accurate surface pressures are indicative of a code's ability to predict lift and moment, but usually do not provide an insight into a code's ability to produce accurate drag. Drag is an aerodynamic parameter of major importance for all flight vehicles and is strongly dependent on viscous effects. Huge sums are expended by aerospace companies in the quest for drag reductions of just a few percent. Yet one finds that today's CFD codes, with certain limited exceptions, are unable to predict drag with an acceptable degree of reliability. They can generally provide qualitative insight concerning whether a given configuration will have "good" or "bad" drag characteristics, but current CFD codes can rarely be depended on to produce a quantitatively accurate prediction of drag, particularly if the drag is based on integration of surface pressures and calculated skin friction. This is a major shortcoming and limitation.

One of the simplest geometries in aerodynamics is a 2-D airfoil, but the ability of today's best CFD codes to predict airfoil drag accurately remains controversial. No two airfoil codes have been shown to predict the same drag over the angle of attack range. Thus, experimental testing of 2-D airfoils continues. Part of the difficulty stems from the uncertainties of turbulence modeling, but a large part of the difficulty stems from the numerical resolution of the detailed features of the flow with sufficient accuracy and reliability to predict drag. The situation is markedly worse for 3-D problems where coarser numerical grids must necessarily be used.

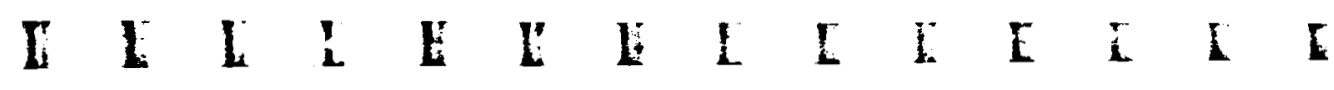


It is important to recognize that a CFD technology capable of predicting accurate surface pressure predictions is far from complete, and that much additional payoff may yet be gained by further improvements in algorithm technology. Possibly the only CFD algorithm technology to date that works acceptably well (within the limitations of its approximations) in nearly all respects for 3-D flow calculations is the higher order panel method technology for simple inviscid linear flows.

Other factors associated with the definition of a CFD capability involve such items as problem setup time, required user expertise, and procedure "tuning." The influence of these factors is perhaps most pronounced in attempts to extend the solution of nonlinear flows involving surface fitted grids to more complex and arbitrary geometries. Such attempts have frequently involved inordinately long and labor-intensive setup times and the accuracy of the computed results depends considerably upon decisions made by the user concerning grid density and perhaps on the "tuning" of artificial dissipation terms. This level of algorithm technology remains far from what is needed.

In spite of the many factors involved in identifying a given capability, the committee attempted to summarize its findings in a simple format in Table 1 . The table shows that CFD methods today can simulate flows about complex geometries with simple physics (e.g., linear flows governed by the Prandtl-Glauert compressibility rule), or flows about simple geometries (e.g., 2-D and axisymmetric) with more complex physics. The areas in the table which combine complex physics and complex geometry show little capability.

The following two-part discussion sets forth a more detailed assessment of capabilities for solving the various flow equations of interest for external aerodynamics. The first part describes capabilities that are used principally for steady flows, although certain of the methods used therein can also be used for the prediction of unsteady flows. The second describes capabilities in use for unsteady aerodynamics.

\section{Capabilities for Steady Aerodynamic Flows}

\section{Linear Methods}

There are several mature codes for calculating flows that can be represented by linear theory - the Prandtl-Glauert simulation

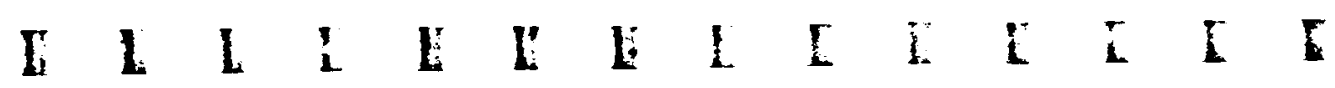




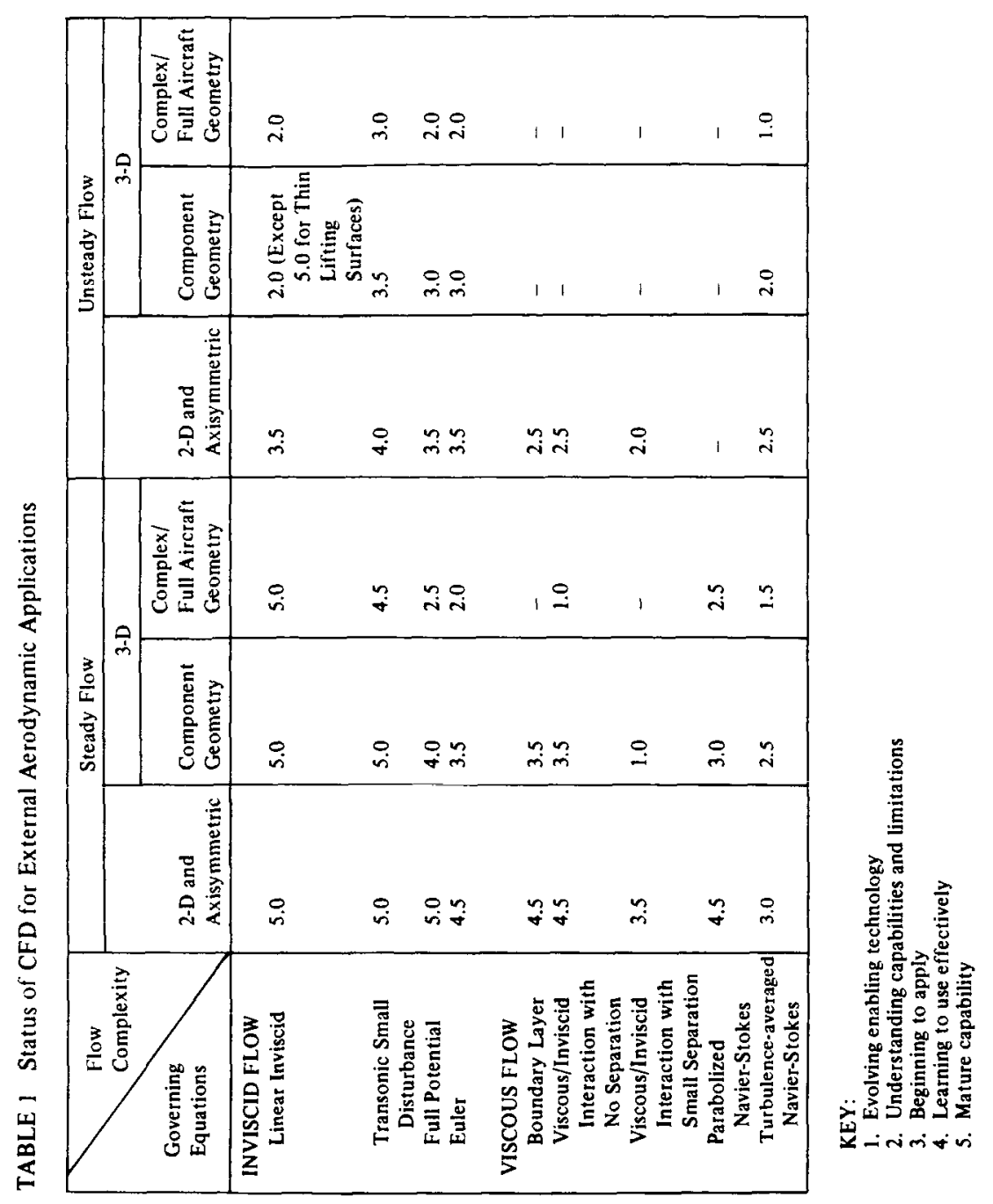

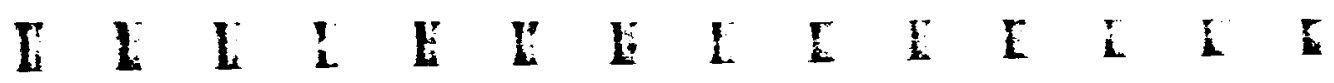

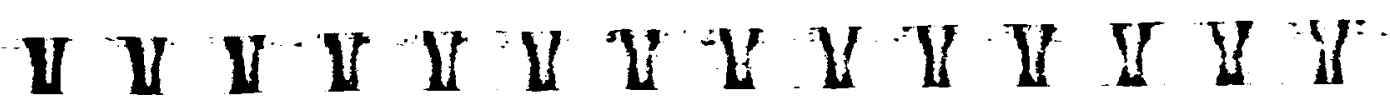


relation. Most of these are limited to subsonic flows, and they all use panel methods. A few panel method codes are available which handle supersonic flows within the framework of the linear equation, and some have been extended to simulate certain limited types of free vortex flows at subsonic speeds.

The panel method codes of today are unique in that they provide a capability for solving the flow about completely general configurations. This is a major virtue which makes them very useful. Once a user gains familiarity with one of these codes, he or she can conduct a host of different applications. Such codes are in regular use within every major aerospace company. The computing cost of carrying out an analysis about a complex configuration is acceptable. Their principal limitation is that they are restricted to simple physics as modeled by the linear Prandtl-Glauert equation.

The general purpose panel method codes are the largest and most complex codes in CFD. As such they require systematic maintenance, and a "home organization" to adapt them to a new computer operating system.

Three-dimensional codes have been coupled with 2-D (strip) direct and inverse boundary-layer codes, and with 3-D boundarylayer codes, but the committee's survey showed little use of coupled viscous analysis. The probable reasons for this are:

1. Most panel method applications are for attached flows in the absence of shock waves, for which viscous effects are not very pronounced.

2. Complex geometries, for which panel methods are used extensively, are restricted by the limitations of the boundary-layer approximation. Boundary-layer methods cannot simulate discontinuous intersections which characterize a geometrically complex configuration. Thus, they are highly restricted in their ability to provide accurate simulations on complex geometries.

One remaining need of the military airplane community is to extend the limited abilities of today's panel methods to better handle the simulation of free vortex flows.

\section{Nonlinear Small-Disturbance Codes}

Several small-disturbance codes exist for calculating transonic flows over general 2- and 3-D geometries with subsonic streams. Most of the codes employ small-disturbance equations which include extra terms to improve the accuracy near highly swept 3-D

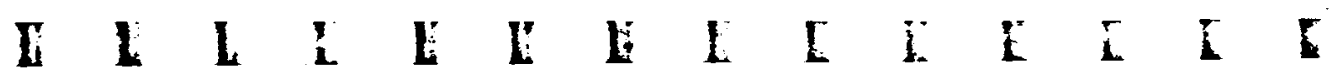

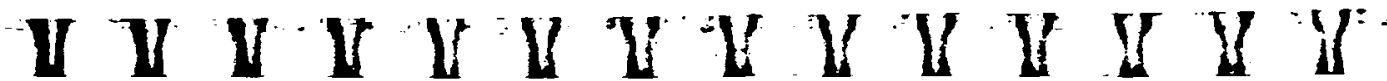


shock waves and to broaden the range of Mach numbers for which the theory can be usefully applied. The codes use standard mixedflow finite difference schemes to solve the approximate potential equation. The use of approximate planar-type boundary condition surfaces permits the use of relatively simple grid structures even for complex 3-D geometries. Usually, Cartesian or polar coordinate type grids are employed with grid embedding to permit grid clustering near important parts of the flow. The grid-embedding techniques employed also allow for the modification of the governing differential equation to better model the flow in each embedded region.

The main advantage of the small-disturbance codes resides in their ability to treat, albeit in approximate fashion, complex 3-D configurations. It is practical to use these codes to compute transonic flows over complete aircraft configurations with combinations of nacelles, pods, pylons, winglets, canards, and with arbitrary fuselage shapes. They can be used to compute component interference effects in the same way as designers using wind tunnels.

Viscous effects are usually treated in a rudimentary way using 2-D strip integral boundary-layer methods in the 3-D applications. This simulates some of the major viscous effects, but is inadequate for the prediction of flow separation and stall.

The accuracy of these codes has not been precisely established. Nevertheless, published results have shown reasonable agreement with experimental data for the pressure distribution for a wide variety of 3-D applications. The results of the computation must be carefully interpreted and combined with other methods to make useful engineering estimates of the aerodynamic forces and moments.

The computer requirements for these codes are modest-less than one-half million words of memory and a few minutes of time on a Class VI computer are required for simple wing-body combinations. The ability to compute the pressure distribution on complex 3-D configurations with reasonable accuracy and modest computer resources make these methods very useful for the aerodynamic design of aircraft. These are relatively mature codes and in all probability will not be developed much further.

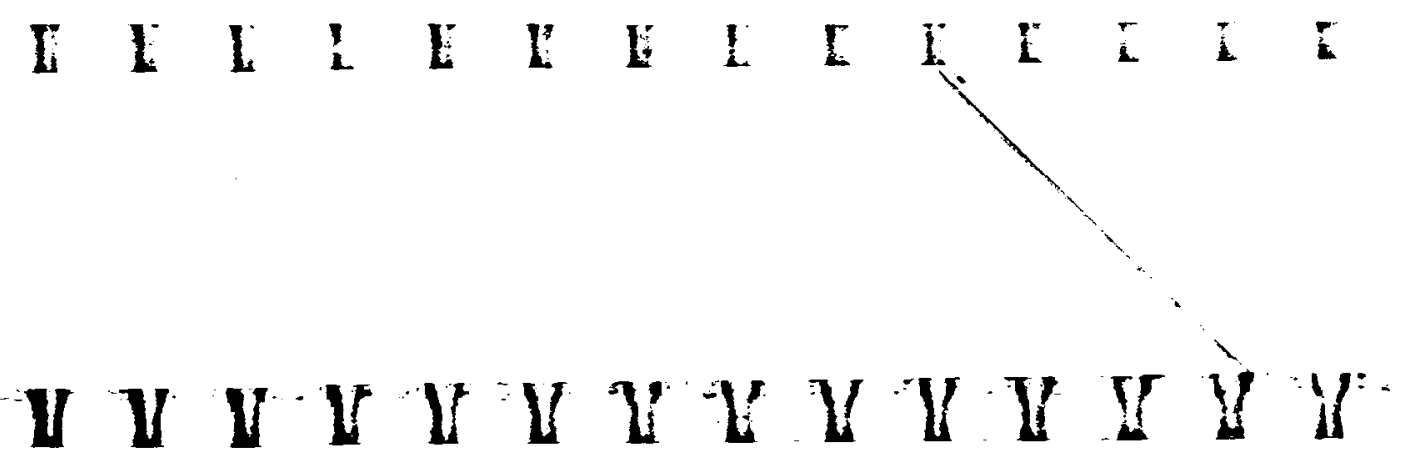


Several full-potential transonic codes exist for calculating the flow about isolated components such as airfoils, wings, and bodies. The algorithms commonly used are finite-difference or finitevolume schemes with surface-fitted grids. Most of these codes are designed for flows with subsonic freestreams.

A lesser number of full-potential codes are available for flows with supersonic freestream that are capable of handling embedded subsonic pockets. These codes use transverse grid planes and are capable of handling more complex geometries, but are not suitable for flows with a subsonic freestream.

With the exception of the codes for supersonic flows, virtually all full-potential codes have evolved down a path that led to "configuration-component" codes, each capable of handling a restricted class of geometrical shapes. This is a different state of affairs than for the world of linear flows, and more restrictive. The capability to handle complex and arbitrary geometry in transonic flow does not exist today. Considerable effort has been and is being spent on extending conventional, surface-fitted grid approaches to more complex geometries by means such as "blockstructured grids," but the practicality, reliability, and usability of those developments have yet to be fully demonstrated.

Other approaches for handling arbitrary and complex geometries should continue to be explored. Among efforts toward that objective are those utilizing triangular and tetrahedron grids, and those embodying a rectangular, nonsurface-fitted grid.

Within the geometrical constraints of today's algorithm technology, the transonic full-potential codes are much used. They are used extensively for wing/body and wing/body/simple-nacelle simulation, particularly by the commercial transport aircraft community. For those applications they are generally coupled with a boundary-layer code. They provide extensive design information in the cruise regime where the flow is attached. They cannot handle separated flows, which are a prime consideration for transport wing design for acceptable handling characteristics at the perimeter of the flight envelope. Their ability to predict drag, a major performance parameter, is also somewhat unreliable. The supersonic codes are restricted to attached flow simulations, which limits their applicability to a small portion of the flight envelope of high-performance military aircraft.
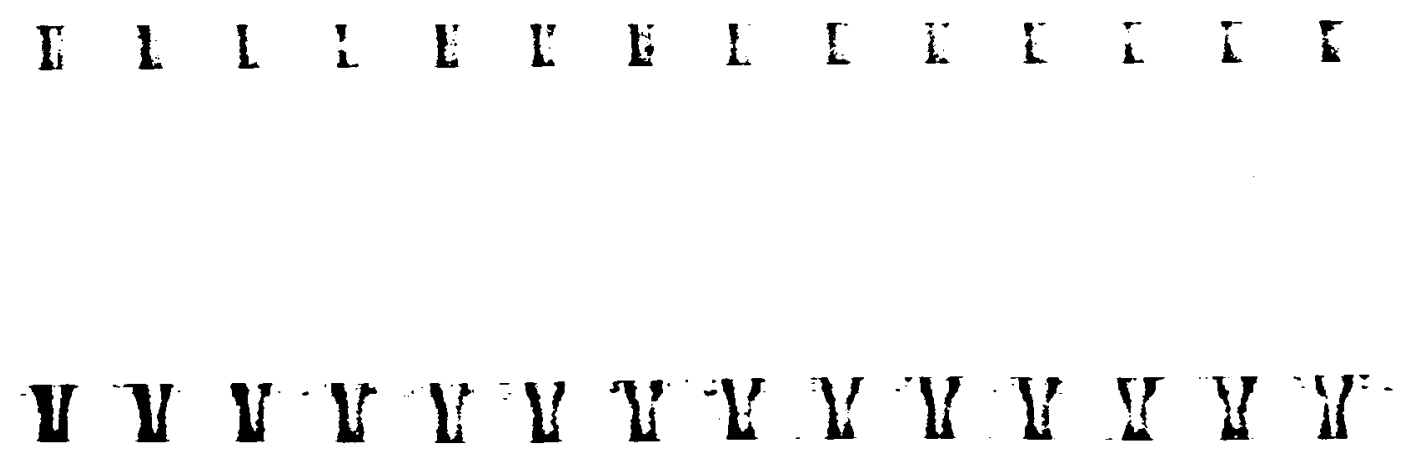
Extension of this class of numerical technology to treat the Euler equations using multiple potential (e.g., Clebsch or vector potential) or stream function methods has been demonstrated in 2-D. It would appear to be feasible and desirable to extend these procedures to 3-D.

\section{Euler Codes}

There has been a strong focus on Euler code development in recent years. Their ability to produce wake position as an output rather than an input, and to model rotational flows and flows containing regions of different total pressure is a major asset not shared by methods based on potential theory.

There are several methods being pursued in operational, 3-D Euler codes that are under continual refinement, and each possesses its own advantages and disadvantages. The most widely used methods fall into two broad classes:

1. Central-difference methods with dissipation terms added to enhance stability and to provide smoothing of shock profiles.

2. Upwind-differenced flux-splitting and total variation diminishing (TVD) methods.

The first class of methods usually requires some tuning to obtain near-optimum values of the coefficients for the added dissipation terms. The optimum values depend on the application, a feature which is undesirable. But the methods are relatively simple compared to those of the second class, which require less tuning. Both classes are extendable to the viscous Navier-Stokes equations.

The existing 3-D Euler codes, which are most widely used in government research laboratories and industry, use nonadaptive, body-fitted grids and most are configuration-dependent. Numerical solutions containing shocks, wakes, and vortex structures are accompanied by excessive diffusion and loss of resolution in the numerics. The algorithms can also produce false entropy due to truncation errors or badly chosen artificial damping terms in local regions where resolution is inadequate; the false entropy is convected downstream and can result in spurious separation and erroneous force coefficients. The computing costs and memory requirements of Euler solvers are higher than for full-potential solvers, but are acceptable provided one has access to a Class VI computer with a large memory.

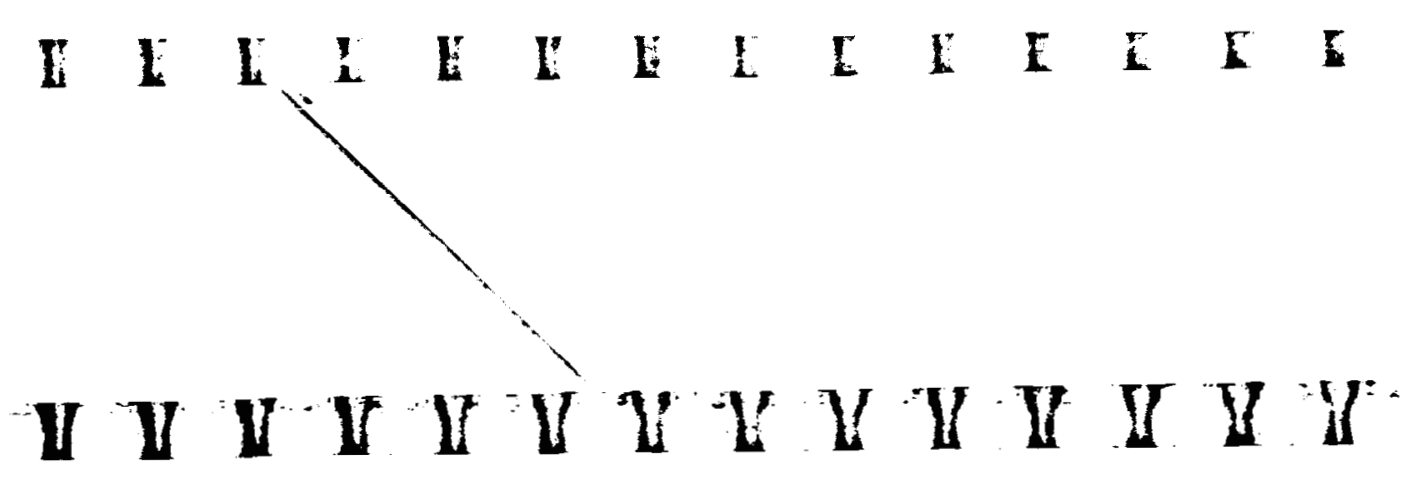


Also, today's operational Euler codes suffer from the same stringent geometry constraints, imposed by the current surfacefitted grid technology, as the full-potential codes. Euler codes exist for wing/bodies, nacelles, actuator disks, detailed propfan blade analysis and other such components, but each is a different code. There is no Euler capability for general configurations, although work is under way involving block-structured grids and triangular and tetrahedron grids, as well as a limited look at nonsurface-fitted grid technology. Here, also, there is a major need for a general configuration capability that does not exist.

Much could be gained from algorithm research aimed at overcoming the limitations of today's computational technology. It could render Euler technology far more effective for the aerospace community, and new developments in Euler technology will probably also contribute directly to Navier-Stokes technology.

Boundary Layer, Viscous/Inviscid Interactions, and Parabolized Navier-Stokes Codes

Several good 2-D and 3-D boundary-layer codes have been refined over the years. Their primary limitations today are (1) accuracy of the turbulence models, (2) concern about the accuracy of crossflow profiles in 3-D integral methods, and (3) the fact that reliable inviscid-viscous interaction schemes have yet to be demonstrated for complex 3-D flows such as corner flows and flows with separation. They are used primarily in airfoil codes, nacelle codes, and in transonic wing codes where their role in the shockwave/boundary-layer interaction phenomena makes their use almost essential. They are also being used to assess the accuracy of emerging Navier-Stokes codes.

Further improvement is needed in the technology of interacting boundary layers with an outer flow, especially 3-D viscous/inviscid interaction methods for the simulation of some types of separated flows. The computational economics of boundarylayer methods are such that there are significant benefits to be gained with these enhancements.

Parabolized Navier-Stokes (PNS) codes are being used extensively to compute steady supersonic and hypersonic flow about streamlined bodies. In the parabolizing approximation the streamwise viscous terms are dropped and a modified streamwise pressure gradient is introduced to allow space marching from upstream

I I L I L I 
initial data. Because only one pass through the grid is required, these procedures are computationally efficient. With the PNS procedure there is no need to couple an inner viscous region with an outer inviscid flow as with conventional boundary-layer methods. However, the PNS codes tend to be difficult to use because they can exhibit numerical instabilities with respect to refinement in streamwise step size. This instability may be avoided with the introduction of semi-elliptic iterative methods but at the expense of increased computer time and memory requirements. Current developmental activities with PNS are centered about the inclusion of real gas effects and finite-rate chemistry.

\section{Navier-Stokes Codes}

Solutions of the 3-D, turbulence-averaged, Navier-Stokes equations for realistic aircraft configurations is the "Holy Grail" of CFD. The cost of Navier-Stokes applications has restricted their use to specialized applications, primarily 2-D flows and limited regions of 3-D flows. Most of the applications so far have used simple algebraic turbulence models with the Reynolds-averaged, thin-layer Navier-Stokes (TLNS) approximation. The TLNS uses body-fitted meshes so that all of the diffusion terms tangential to the solid boundaries can be conveniently dropped from the equations, retaining only those normal to the boundaries. This eliminates the need to treat meshes that are fine enough in all directions to resolve properly all the viscous derivatives. A crude estimate of the number of mesh points required to obtain a reasonably accurate solution of the TLNS is twice the number of mesh points as for a good Euler solution.

The point has been reached where the industry is beginning to experiment with Navier-Stokes codes, and this is leading to a realization of the immense capability that is to come. Algorithm development for 3-D Navier-Stokes equations is still in a primitive state. All of the problems with Euler solvers carry over to the Navier-Stokes, but are often masked by the viscous effects or inadequate turbulence models; hence, they may not be so clearly evident. Geometrical capabilities are even more limited than for the Euler solvers, partly because of the need for resolution of vastly differing length scales.

The issues of Navier-Stokes should be attacked on several fronts. Merely awaiting the next generation of supercomputers

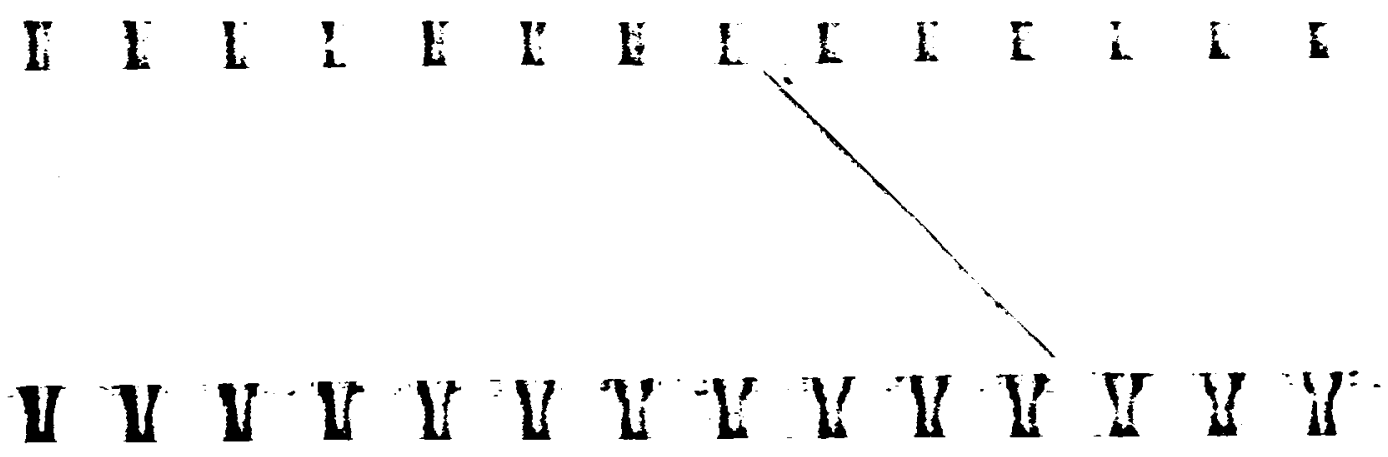


is not enough. Computer time required for present TLNS codes for even an isolated wing range from 2 to 8 hours on a Cyber 205 or CRAY X MP. Improvements in speed of convergence must be sought to reduce the required number of iterations to reach a steady state from thousands down to hundreds. Algorithms are needed that do not suffer serious loss in performance and robustness to high aspect ratio meshes and artificial viscosity. Even more compelling than in the case of Euler solvers is the need for efficient, solution-adaptive meshes that can track and resolve shocks, vortex structures, boundary layers, wakes, and free shear layers. Work to date has been mostly with global, nonadaptive, body-fitted grids for single components such as an airfoil, wing, or isolated nacelle. However, promising research has shown the possibilities afforded by overlapping embedded grids, zonal bodyfitted grids, and finite-element-type tetrahedral grids.

Turbulence modeling will become a major issue for NavierStokes solvers, but with today's algorithms the various errors associated with numerics are probably a larger issue in need of resolution. When those sources of error are resolved, then turbulence modeling will become the limiting factor.

\section{Capabilities for Unsteady Aerodynamic Flows}

The principal applications of CFD methods for unsteady flows within the aerospace industry have been associated with structural dynamics, flutter, and active controls. To date there has been less emphasis on the development of methods for unsteady aerodynamics than for steady flows. Several of the computational methods in common use for steady aerodynamics can be used in a timeaccurate, unsteady mode. However, in practice many of these are never used for unsteady applications, the probable reason being that the computing costs required to carry out time-accurate calculations for the wide range of Mach numbers, angles of attack, control surface motions, and dynamic pressures that must be examined for a comprehensive flutter analysis remain beyond the normal realm of affordability.

Linear Methods

There are several programs for calculating unsteady flows based on linear theory. Subsonic kernel function methods are

I $\quad$ I L L 
available for thin lifting surfaces, i.e., wings and tails. Kernel methods are available to analyze wings with oscillating control surfaces. Doublet lattice methods are available for modeling flows past somewhat more complex geometries including pylons and nacelles. Doublet lattice codes that have been modified using transonic static lift curve slope data (obtained from experiment or computations which include viscous corrections and separation) are used to extend this capability to the nonlinear range.

Supersonic lifting surface theory has also been implemented for thin lifting surfaces. These methods are also based on small perturbation theory.

Surface panel methods have been developed to compute flows about bodies of arbitrary shape in subsonic flow. Attempts to extend them to the transonic and supersonic speed ranges are ongoing. The shuttle orbiter and the shuttle launch stack are examples of complex configurations that have been analyzed using panel methods. It is possible to do time-domain or frequencydomain (with complex frequencies) analyses. One current thrust is to combine panel methods with a hybrid vortex method to treat vortex-dominated flows. Also being developed is a scalar and vector potential method for treating viscous, compressible flows.

\section{Transonic Small-Disturbance Codes}

Several codes exist for calculating 2-D transonic small-disturbance flows. They employ finite difference as the form of discretization. Some use Alternating Direction Implicit (ADI) procedures to advance the solutions in time. Others are available for solving the low frequency and the general frequency harmonic equations. Codes of this latter type are inexpensive to run and have been used to compute 2-D transonic small-disturbance flows.

Three-dimensional small-disturbance methods that advance the solution in time have been developed. The current capability is for thin clean wings. Some work has been done to add body, tail and canard, and tip store capability. Currently, the codes are computationally inefficient, expensive to run, and require small time steps to maintain stability.

Methods for computing harmonic transonic linear perturbations about nonlinear steady states are available for 2-D and 3-D flows. In 3-D, such methods have thin, clean wing capability. When iterative techniques are used to invert the matrices, a limit

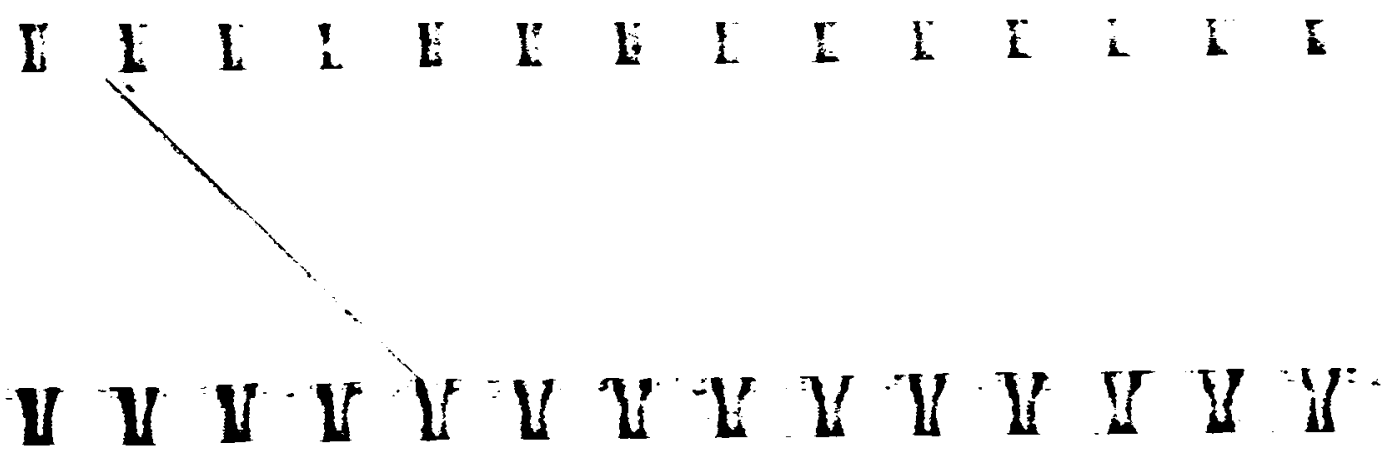


on the frequency of the unsteady motion is encountered. This limit is a function of the freestream Mach number and the size of the computational domain. Use of a direct matrix inversion approach serves to overcome this limitation, except that the cost and storage can become prohibitive in 3-D problems.

\section{Full-Potential Codes}

Several time-marching full-potential methods are available for calculating 2-D and 3-D flows. Some early 2-D codes solved the nonconservative flow equation, but later codes almost exclusively solve the conservative equation-usually in a body-fitted coordinate system. Two approaches for implementing boundary conditions are used-one in which transpiration conditions are applied on the mean surface and one in which a flow tangency condition is applied on the body boundary in a grid system that instantaneously conforms to the body. The relative merits of the two approaches have not been clearly established.

Three-dimensional capability depends on the freestream Mach number. The capability to compute flows past complete vehicles is available for supersonic flows. For subsonic and transonic flows, codes that can do wing and body analysis are mature. An effort is under way to develop a single code that can compute subsonic, transonic, and supersonic flows past full configurations and that includes aeroelastic analysis capability. A 3-D method that includes rotational flow effects is also available for wings.

Since the 3-D potential methods use body-fitted coordinate systems, one of the most significant problems that has to be addressed is gridding about complex configurations.

A drawback of potential methods, which is experienced particularly in 2-D applications, is the occurrence of nonunique solutions that can be obtained when shocks are in the flow field. Progress has been made in solving this problem by accounting for entropy jumps across shocks in potential flow. Such entropy corrections to potential flow are available for 2-D and 3-D methods.

\section{Euler Codes}

Euler codes have received increased attention in the past few years. Two-dimensional Euler codes are available that use timedependent conformal grids. There is a capability in 3-D for obtaining solutions for complete aircraft in supersonic flows. At

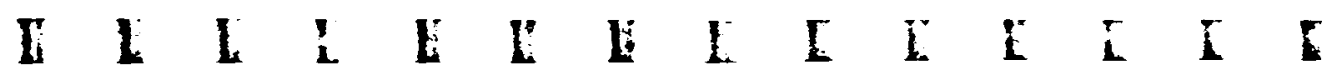


transonic speeds, state-of-the-art methods are limited to wing and body configurations. A method for including tail effects is under development. As with full potential, a major consideration is grid generation for complex configurations.

Generally, for the number of cases that must be run to do a typical aeroelastic analysis, Euler codes are not yet practical for everyday engineering use.

\section{Boundary-Layer Codes}

Many inviscid codes are coupled with boundary-layer models to simulate viscous effects. An unsteady, integral boundary-layer code has been coupled with transonic small-disturbance codes (in 2-D) with reportedly good results, but it is expensive to run. A 3-D unsteady finite difference boundary-layer code is under development but has not yet been coupled with any inviscid flow code; it has been run for a flat plate.

Other methods involve coupling unsteady inviscid methods with steady boundary layers at each instant in time. In 2-D, inviscid codes have been coupled with integral boundary-layer methods. In 3-D, the inviscid methods have been coupled with a 2D strip boundary layer at each span station and at each time step. The overall accuracy of such approaches has not been determined.

\section{Navier-Stokes Codes}

Two-dimensional Navier-Stokes codes exist for subsonic, transonic, and supersonic calculations. Codes for solving the full turbulence-averaged equations and the thin layer approximation to the equations exist for wing alone and for a blended wing and body (e.g., shuttle orbiter wing and fuselage configuration). One significant problem is the turbulence model-most codes still use the simple flux-gradient concept. The number of grid points needed to resolve the viscous region also makes storage a major problem for other than simple configurations. In practice, Navier-Stokes methods are computationally prohibitive except for perhaps very specialized applications.

\section{FINDINGS}

CFD offers great potential in many areas of fluid flow and to a large degree this potential is now being realized in aircraft

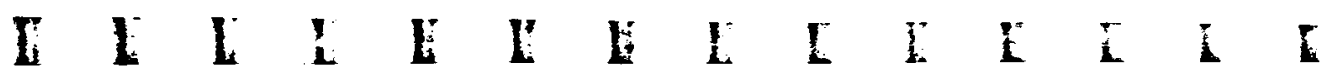


design. All major aerospace companies have acquired, or will soon acquire, supercomputers and the CFD research supported by these companies is growing. Within the commercial transport aircraft community the application of CFD has become an integral part of the aerodynamic design process. The more complex flows encountered by high-performance military aircraft have proven more difficult, but a few of these are now beginning to succumb to computational analysis. However, much remains to be done.

Today, CFD methods can simulate flows about complex geometries with simple physics, or about simple geometries with more complex physics, but they cannot do both. The most pressing need is for algorithm development that will lead to practical methods for solving the nonlinear flow equations-full-potential, Euler, and the various forms of Navier-Stokes equations-about arbitrary configurations. The emphasis to date has been on learning how to solve these nonlinear flow equations for simple geometries about which orderly, surface-fitted, quadrilateral grids can be fitted, and progress has been made along that path. Today's algorithms are cheaper, more accurate, and more robust that those of yesterday, but they are still unsatisfactory in many respects for Euler equations and in most respects for the Navier-Stokes. Useful codes have been produced, but better algorithm technology could multiply their usefulness many times over.

A greater focus is required today on innovative algorithm research to deal with complex geometries in a practical, usable, and reliable manner. The panel methods for linear flows are in a class by themselves in this regard and should serve as a model for the simulation of nonlinear flows. Examination of typical panel method applications in industry reveals the immense value of being able to readily simulate flows about very complex geometries replete with components of widely varying length scales. The more general of those codes are structured as arbitrary boundary value problem solvers, and the codes themselves contain no concept of specific airplane components such as wing, body, and tail. They can truly solve any properly posed boundary value problem of the governing differential equation with great flexibility.

This generality is what should be sought with the nonlinear flow solvers. Much effort is currently directed toward adaptations of quadrilateral-based, surface-fitted grids involving block structuring as a means to extend nonlinear flow solvers to more geometrically complex applications. However, it is uncertain whether

I L L I L E I

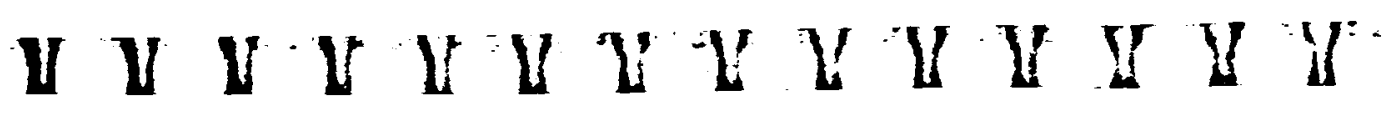


will lead to practical, complex geometry capability. Innovation is needed in the search for breakthrough in numerical algorithms aimed at solving geometrically complex problems involving many length scales.

Codes solving linear flows, the boundary-layer equations, the full-potential equations, Euler equations, and the various forms of the turbulence-averaged Navier-Stokes equations all have a role to play in the aircraft industry and will continue to do so for the foreseeable future. They are all important. The advent of more capable supercomputers is not reducing the impact of economic limitations. Rather, the opposite is happening. We seek to solve larger and more complex problems whenever a new generation of computer becomes available, and total expenditures on computing continue to increase. The user community will continue to need and use codes solving the simpler equations when allowed by the physics, and will use codes solving the more complex physics (which are usually more expensive to set up and run) only when the complexity of the physics requires it. The engineering application of CFD is dictated by economics.

In addition to the inability of today's nonlinear solution methods to deal effectively with complex geometries, another significant weakness involves the various shortcomings in the basic algorithm technology for all but panel methods. The drag prediction abilities of all nonlinear 3-D codes are unreliable at best, and for some they are so unreliable that computed drag is totally ignored. Most of that is traceable to the inefficient numerical algorithm or to the prohibitive cost associated with use of the high grid densities needed for accurate numerical resolution. Turbulence modeling will certainly become a limiting factor in the future, but until the numerical accuracy issue is solved, the limitations of turbulence modeling will be difficult to assess.

The greatest need for algorithm improvement is in the technology for Euler and Navier-Stokes solvers, and in fact they are closely related. Advances in one should lead to advances in the other. Major improvements are needed in spatial accuracy and solution adaptability, convergence reliability, and convergence rates.

The committee observed that an increasing number of issues related to computer science are appearing in the development of advanced computational capabilities. As nonlinear codes begin to treat more complex 3-D configurations, the issues of code vectorization and multitasking, treatment of large data bases, automatic

\section{I $I$ I I I I}


analysis of computed generated results, 3-D graphic displays, and surface and grid generation become increasingly important. A blend of skills in these areas is becoming increasingly necessary for the development of efficient and effective computational capabilities.

A further area of concern for the user of CFD capability is the verification of accuracy and applicability of codes for practical design applications. The issue here is the ability of the equations and the solution algorithms to represent the physics for flows of interest. Industry must develop a high level of confidence in the validity of CFD solutions for making management decisions before CFD is accepted as a valid design tool. Such confidence is gained by careful comparison of solutions with detailed experimental data.

Verification of flow solvers is a vital part of the CFD development process. The effects of grid density, numerical dissipation, turbulence modeling, and convergence reliability need to be understood and related to the flow physics by comparison with reliable experimental data.

\section{RECOMMENDATIONS}

Stronger emphasis is recommended on the development of more advanced algorithm technology, particularly for the Euler equations and the various forms of the turbulence-averaged Navier-Stokes equations. Two objectives must be sought. One is technology to enable computation of flows about truly arbitrary and often complex geometries in a manner that is practical and for which accuracy is predictable. Such technology must include means for resolving phenomena that occur at the differing length scales found in nonlinear flows such as thin boundary layers, shock waves, free shear layers, exhaust plumes, and regions of separated flow. The other objective is for major improvements in spatial accuracy, convergence reliability, and convergence rates. The committee believes that order-of-magnitude improvements are possible and should be aggressively sought.

It is further recommended that continuing emphasis be placed on verification and validation of flow models and solution algorithms by comparison with detailed experimental data. Such comparisons are a vital part of establishing a mature CFD capability.

I I L I L L E

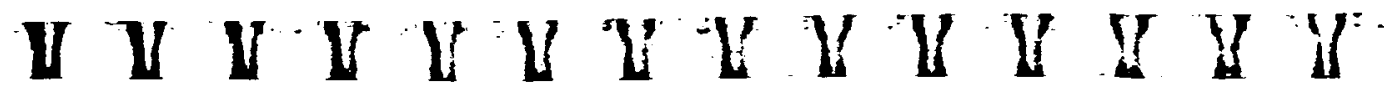




\section{Hypersonic Applications of CFD}

\section{OVERVIEW}

During the past three decades, the level of research and development in hypersonic flow phenomena has changed drastically. Initially, this effort experienced an intense surge from the mid1950 s through the 1960s; then a near demise from the 1970s through the early 1980s; and, in the past few years, the beginning of a resurgence as a vital technology for potential NASA and Department of Defense (DOD) missions in the 1990s and beyond. The initial thrust centered around the Intercontinental Ballistic Missile (ICBM) and Apollo programs. It was intensified by the newness of many hypersonic flow problems encountered at that time. To overcome these, a major national research and development effort for about 15 years was required. The subsequent comparatively quiescent period of hypersonic research in the 1970s centered mainly on the shuttle program and the Jupiter atmospheric entry probe. A resurgence of interest in hypersonics has emerged, driven by advanced new applications such as: (1) aero-assisted orbital transfer vehicles (AOTV) that achieve payload economy by passing through the earth's upper atmosphere to change orbit; (2) transatmospheric vehicles (TAV) that take off from earth or a conventional aircraft to enter near-earth orbit, then re-enter the atmosphere with cross-range capability to land on an airstrip; (3) hypersonic aircraft anticipated to require propulsion by supersonic combustion ramjets; and (4) the Strategic Defense Initiative that potentially involves hypersonic flow phenomena such as those associated with signal discrimination

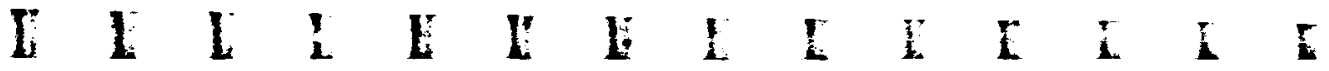


and sensor design. Together, these new applications encompass a very broad range of hypersonic physics, and bring into prominence some new aspects of hypersonic CFD which, in past applications, have only been partially dealt with, or not dealt with at all.

Two major technological phenomena rising into prominence in these new applications are nonequilibrium chemistry and radiative heating. These arise because vehicles such as AOTV and TAV must operate at much higher altitudes than the earlier hypersonic vehicles. Although peak heating rates and maximum aerodynamic forces are developed in the $40-\mathrm{km}$ to $50-\mathrm{km}$ altitude range, these vehicles will spend greater time intervals in the $50-\mathrm{km}$ to $100-\mathrm{km}$ region where the flow characteristics are less well understood.

For such low densities and high velocities, the molecular reaction rates within a fluid element do not keep pace with the rapid flow changes. In consequence, internal molecular energy modes, chemical dissociation, species ionization, and molecular radiation are all out of equilibrium. This essential nonequilibrium character complicates extensively the numerical computation of flow fields. It also has a significant impact on the relative roles of computation and experimentation in the vehicle design process.

Experimental simulation of a hypersonic flow field in which nonequilibrium reaction-rate chemistry is important would require the air density, flight velocity, and vehicle scale all to be reproduced simultaneously. This is not possible in existing ground-based experimental facilities. The simultaneous simulation of density, velocity, and scale, however, is possible in computer flow simulations. As a result, CFD is anticipated to be the primary tool for providing the extensive flow simulation information required in the design process of new hypersonic vehicles operating in the upper atmosphere; and flight tests may be the primary means of providing the necessary validation of the computational methods. This situation is totally different from that of earlier hypersonic vehicles designed on the basis of chemical equilibrium flow, and from that of hypersonic aircraft operating in the lower atmosphere; for these, small-scale laboratory experiments together with corrections for "real gas" effects can provide realistic flow simulation information.

I 1 L L I I 


\section{DEPARTURES FROM \\ CONVENTIONAI AIRCRAFT CFD}

Hypersonic CFD for flight in the upper atmosphere is not merely a modest extension of conventional aircraft CFD, but is an intricate technology involving new complexities that do not exist at the Mach numbers of aircraft flight. Some of these complexities are not well-understood, and the necessary data base for many others does not yet exist.

To begin with, the useful equation sets for numerical computation differ considerably. For hypersonic applications small perturbation approximations, for example, are virtually useless, and even the Euler approximation with coupled boundary layer is of relatively limited use because of the inaccuracy of the boundary layer approximation in hypersonic flows that are strongly dominated by viscous effects. Some of these flows can require governing equations sets quite different from the conventional Navier-Stokes equations.

A major complication is that the continuum fluid model is not realistic for all hypersonic flight conditions of interest, whereas it is not in doubt for the flight conditions of conventional aircraft. Although the peak heating and principal maneuvering of advanced hypersonic vehicles may occur at altitudes within the continuum regime, other important aspects of their flight occur at higher altitudes where the mean free path of the molecules becomes of sufficient magnitude relative to the vehicle dimensions that the continuum model breaks down. Here particulate flow simulations must be employed wherein the motion of a large number of molecules is computed, such as in the direct simulation Monte Carlo method. Thus, both particulate and continuum numerical flow simulation methods are required for hypersonic CFD in the upper atmosphere, whereas only continuum simulations are needed for conventional aircraft CFD.

Another complication is that the conventional continuum Navier-Stokes equations of motion-which are not seriously in doubt for ordinary aircraft flight conditions-can be unrealistic for hypersonic flight in the upper atmosphere, and are of uncertain accuracy in the lower atmosphere. Two assumptions embodied in the Navier-Stokes equations may become either uncertain or unrealistic under hypersonic flight conditions. One is that of a linear

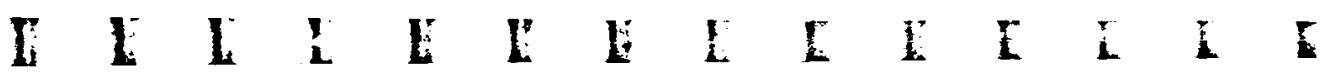


relationship between viscous stress and strain rate. This assumption is known to be valid for small departures from equilibrium; but in hypersonic flows these departures can be very large indeed, and thereby place doubt on the accuracy of this assumption of linearity.

The second questionable assumption is the "Stokes hypothesis" - that although viscous stresses exist when fluid layers slide relative to each other without density change, no such stress exists when the density changes without fluid layers sliding relative to each other. For hypersonic flows the density of a flowing particle can change with extreme rapidity, and this brings Stokes' hypothesis into serious question. In particular, this hypothesis precludes taking into account in the equations of motion certain physical processes that are known to exist, but that are not represented by any term in the conventional Navier-Stokes equations (such as the processes of irreversible molecular energy exchange between internal and translational modes produced by inelastic molecular collisions). Modifications to the Navier-Stokes equations which would account for such processes, if indeed needed, have not yet been established.

The lack of realism of the conventional Navier-Stokes equations becomes clear for flight conditions in the upper atmosphere wherein the shock-wave thickness itself is not small compared to the shock stand-off distance. On the nose of the shuttle orbiter, for example, this situation exists at altitudes of about $75 \mathrm{~km}$ and higher. Under such conditions the CFD code must integrate through the shock-wave profile. It has long been known, however, that the Navier-Stokes equations are incapable of doing this accurately at Mach numbers above about 2 . To do so accurately at hypersonic Mach numbers will require that continuum equations of motion be developed with a higher degree of realism than the conventional Navier-Stokes equations.

Probably the greatest complication and departure from conventional aircraft CFD is the massive amount of molecular physics data needed as inputs in hypersonic CFD, whether of the continuum or particulate type simulation. Quantitative input data are required, for example, on reaction rates for all the possible atomic, molecular, and ionized species of air (as much as 11 different species and over 50 different reaction rates). Likewise, similar data are required on the vibrational and radiation excitation rates, since radiative heating can dominate convective heating
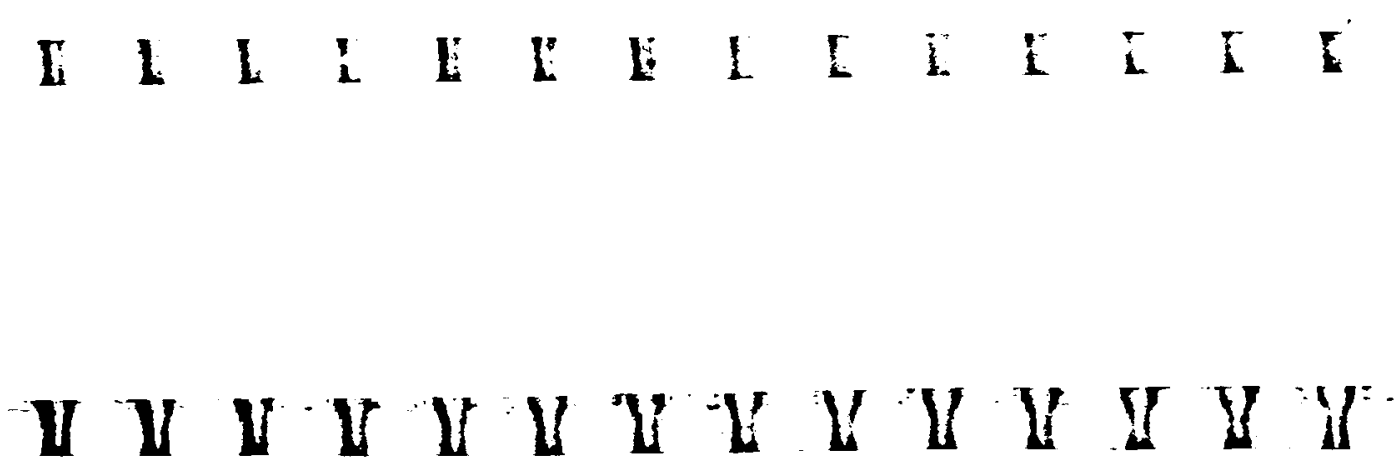
in hypersonic high-altitude flight. Moreover, important gas and surface chemical reactions occur, and data on these rates also are required.

Much of this overall molecular physics data base is, unfortunately, not available at present for the extreme temperatures encountered in hypersonic shock layers. Almost all the available reaction rate constants for air, for example, were measured over 25 years ago and only at the lower end of the temperature range of interest. It will be a sizable undertaking to provide the necessary molecular physics data base.

\section{CFD CAPABILITIES IN HYPERSONICS: UPPER AND LOWER ATMOSPHERE}

The degree to which CFD can contribute to hypersonic design applications is quite different for the two extremes of flight in the lower and upper atmosphere. For flight in the lower atmosphere with equilibrium air chemistry, the feasible applications would be generally similar to those discussed previously for conventional aircraft. The problems of transition and turbulence, ubiquitous to conventional aircraft, are also fundamental to hypersonic aircraft since they can affect aerodynamic heating and payload weight significantly.

Hypersonic aircraft configurations, however, are geometrically less intricate than conventional aircraft, and are thus more amenable to realistic $3-D$ grid generation. This characteristic, together with the ability of CFD to assess real gas effects not generally simulated in experimental facilities, leads to the expectation that CFD applications to hypersonic flight in the lower atmosphere will be relatively more extensive than the corresponding applications to conventional aircraft flight. In particular, Navier-Stokes CFD simulations contribute to insight and understanding by revealing areas of flow separation and intense heating, locations where the bow shock wave intersects other portions of the vehicle, and potential problems of inlet-airframe interaction. These simulations also contribute to the evaluation of innovative concepts by providing such information as the real gas effects on lift-drag ratio, stability, and aerodynamic heating. The applications for the lower atmosphere also extend to some design details that are affected by real gas corrections to hypersonic wind tunnel data.

I $\quad$ I L L 
The other hypersonic extreme of flight in the upper atmosphere may not be a major design driver since peak aerodynamic forces and heat rates occur at lower altitudes. However, the importance of CFD as a basic flow analysis tool is expected to be very much greater. This is because of the inability of existing ground-based experimental facilities to duplicate vehicle scale. Thus, hypersonic CFD for this very high altitude environment is anticipated to be the primary source of flow simulation information for all aspects of application-insight and understanding, evaluation of innovative concepts, and design detail. Because no way is known, other than CFD and full-scale flight experiments, to simulate fully the reaction-rate dependent hypersonic flow in the upper atmosphere, CFD is clearly the primary tool for the design of such vehicles.

\section{CURRENT STATUS OF HYPERSONIC CFD}

In assessing the current status of hypersonic CFD a matrix structure of fluid-flow model versus flow-field complexity was selected that is different from that used for conventional aircraft CFD. Moreover, different matrix structures are appropriate for the two different extremes within the overall scope of hypersonic flow computation. The essential elements involved in CFD of hypersonic aircraft, for example, are quite different from those of AOTV because of the higher velocities and much higher altitudes involved with AOTV. A list of some of these contrasting differences appears in Table 2. In a sense, flight altitude affects the nature of hypersonic CFD to a major degree in an analagous way that Mach number affects conventional aircraft CFD. Consequently, the two extremes are used to characterize the present overall status of hypersonic CFD. One extreme is for hypersonic aircraft operating in the range of a 0 - to $40-\mathrm{km}$ altitude, and the other is for upper atmosphere vehicles such as AOTV operating at altitudes of about $80 \mathrm{~km}$ and higher.

An assessment has been made of the current status of hypersonic CFD based on inputs received from the committee's survey of representative CFD codes developed by industry, government agencies, and the university community. Approximately 47 individual inputs on hypersonic codes were received to form the main basis of this survey. In a few cases these inputs were supplemented by committee members' personal knowledge of existing codes for

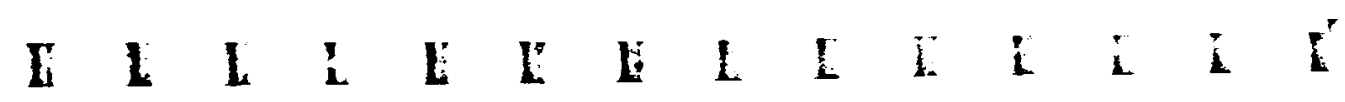

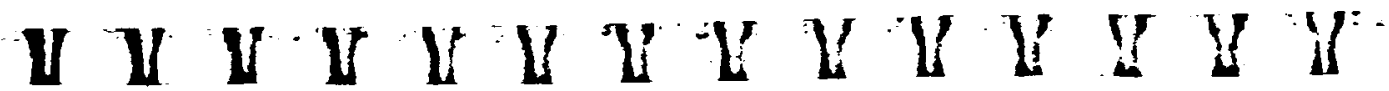


TABLE 2 Contrasts within Hypersonic CFD

\begin{tabular}{|c|c|c|}
\hline & $\begin{array}{l}\text { Lower Atmosphere } \\
\text { Hypersonic Aircraft }\end{array}$ & $\begin{array}{l}\text { Upper Atmosphere } \\
\text { AOTV }\end{array}$ \\
\hline Altitudes & $0-40 \mathrm{~km}$ & $80-110 \mathrm{~km}$ \\
\hline Reynolds Number & High & Low \\
\hline Flow Type & $\begin{array}{l}\text { Turbulent and } \\
\text { Transitional }\end{array}$ & Laminar \\
\hline Flow Model & Continuum & Continuum and Particulate \\
\hline Air Chemistry & Equilibrium & Non-Equilibrium \\
\hline Principal Heating & $\begin{array}{l}\text { Turbulent/Laminar } \\
\text { Convection }\end{array}$ & Radiation, Convection \\
\hline $\begin{array}{l}\text { Molecular Physics } \\
\text { Input }\end{array}$ & None & Extensive Data Base Needed \\
\hline $\begin{array}{l}\text { Surface Boundary } \\
\text { Conditions }\end{array}$ & No Slip & $\begin{array}{l}\text { Slip with Momentum, Energy, } \\
\text { and Chemical Accommodation }\end{array}$ \\
\hline $\begin{array}{l}\text { Shock Wave } \\
\text { Thickness }\end{array}$ & Negligible & $\begin{array}{l}\text { Sizeable Fraction of } \\
\text { Detachment Distance }\end{array}$ \\
\hline
\end{tabular}

which individual inputs were not received. Since few existing codes compute nonequilibrium radiative heating, this important aspect of hypersonic CFD for flight in the upper atmosphere is not reflected in the data presented in Tables 3 and 4 . These tables also do not reflect the development status of special numerical problems that may arise at very high Mach numbers such as with temperature overshoots in shock capturing techniques artificially influencing chemical reactions.

This assessment was quantified by identifying the present stage within the code development cycle (described earlier) corresponding to each hypersonic flow-field model and each degree of flow-field complexity considered. As before, these range from grade 1 for codes in an initial research phase of development, to grade 5 for codes in a mature engineering design phase. The assessment results are summarized in Table 3 for hypersonic flight in the lower atmosphere (e.g., hypersonic aircraft) and in Table 4 for the upper atmosphere (e.g., AOTV type vehicles). Within each box of these matrix tables, further identification is noted as to whether a given stage corresponds to a code for a perfect gas (PG), or to equilibrium air chemistry (EC), or to nonequilibrium air chemistry (NEC). This supplementary notation is indicated because a code for a given hypersonic flow model and given degree of flow complexity might be, for example, in grade 3 for a perfect

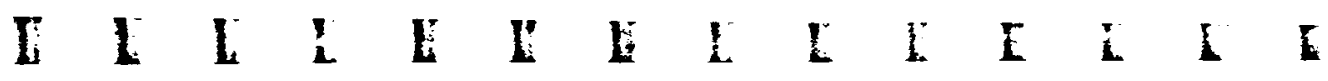


TABLE 3 Status of CFD Capability-Hypersonic Lower Atmosphere

\begin{tabular}{|c|c|c|c|c|c|}
\hline \multirow{3}{*}{$\begin{array}{l}\text { Governing } \\
\text { Equations }\end{array}$} & \multicolumn{5}{|c|}{ Geometry } \\
\hline & \multirow{2}{*}{$\begin{array}{l}\text { 2-D or } \\
\text { Axial } \\
\text { Sy mm. }\end{array}$} & \multicolumn{3}{|c|}{ 3-D } & \multirow{2}{*}{$\begin{array}{l}\text { 3-D } \\
\text { Separated } \\
\text { Flow }\end{array}$} \\
\hline & & $\begin{array}{l}\text { Blunt } \\
\text { Body }\end{array}$ & $\begin{array}{l}\text { Lifting } \\
\text { Vehicle }\end{array}$ & $\begin{array}{l}\text { Lifting } \\
\text { and Inlet }\end{array}$ & \\
\hline Euler & $\begin{array}{l}5 \mathrm{PG} \\
4 \mathrm{EC} \\
1 \mathrm{NEC}\end{array}$ & $\begin{array}{l}4 \text { PG } \\
4 \text { EC } \\
2 \text { NEC }\end{array}$ & $\begin{array}{l}3 \text { PG } \\
3 \text { EC } \\
1 \text { NEC }\end{array}$ & & N.A. \\
\hline Euler Plus Coupled B.L. & 3 PG & 3 PG & 3 PG & & N.A. \\
\hline Laminar & $3 \mathrm{EC}$ & $3 \mathrm{EC}$ & $\begin{array}{l}3 \mathrm{EC} \\
1 \mathrm{NEC}\end{array}$ & & \\
\hline Euler Plus Coupled B.L. & 3 PG & 3 PG & 3 PG & & N.A. \\
\hline Turbulent & $3 \mathbf{E C}$ & $3 \mathrm{EC}$ & $3 \mathrm{EC}$ & & \\
\hline $\begin{array}{l}\text { Parabolized } \\
\text { Navier-Stokes }\end{array}$ & $\begin{array}{l}4 \text { PG } \\
3 \text { EC } \\
2 \text { NEC }\end{array}$ & N.A. & $\begin{array}{l}3 \text { PG } \\
2 \text { EC } \\
1 \text { NEC }\end{array}$ & 2 PG & N.A. \\
\hline $\begin{array}{l}\text { Thin-Layer or Shock- } \\
\text { Layer Navier-Stokes }\end{array}$ & $\begin{array}{l}4 \mathrm{PG} \\
4 \mathrm{EC} \\
2 \mathrm{NEC}\end{array}$ & $\begin{array}{l}3 \text { PG } \\
3 \text { EC } \\
1 \text { NEC }\end{array}$ & $\begin{array}{l}2 \text { PG } \\
2 \text { EC } \\
1 \text { NEC }\end{array}$ & 1 PG & 2 PG \\
\hline Navier-Stokes & $\begin{array}{l}4 \mathrm{PG} \\
3 \mathrm{EC} \\
1 \mathrm{NEC}\end{array}$ & $\begin{array}{l}4 \text { PG } \\
3 \text { EC } \\
1 \text { NEC }\end{array}$ & $\begin{array}{l}2 \text { PG } \\
1 \mathrm{EC} \\
1 \mathrm{NEC}\end{array}$ & $1 \mathrm{PG}$ & $\begin{array}{l}2 \mathrm{PG} \\
1 \mathrm{EC} \\
1 \mathrm{NEC}\end{array}$ \\
\hline
\end{tabular}

KEY:

1. Evolving enabling technology

$P G=$ perfect gas

2. Understanding capabilities and limitations

3. Beginning to apply

$E C=$ equilibrium air chemistry

4. Learning to use effectively

NEC = non-equilibrium air chemistry

5. Mature capability

N.A. = not applicable

gas, yet only in grade 1 for air undergoing nonequilibrium chemical reactions.

Table 3, representative of hypersonic fight in the lower atmosphere, indicates that the present status of CFD is reasonably well advanced regarding the degree of flow-field complexity and the degree of completeness of the governing equations solved by the codes. Such is to be expected since this case is only a modest extension of supersonic aircraft CFD wherein real gas, equilibrium, thermodynamic properties of air are used in place of a perfect gas. For some of the simpler degrees of flow-field complexity, the

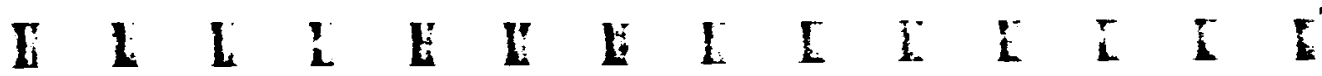


TABLE 4 Status of CFD Capability-Hypersonic Upper Atmosphere

\begin{tabular}{|c|c|c|c|c|}
\hline \multirow{3}{*}{ Equations } & \multicolumn{4}{|c|}{ Geometry } \\
\hline & \multirow{2}{*}{$\begin{array}{l}\text { 2-D or } \\
\text { Axial } \\
\text { Symm. }\end{array}$} & \multicolumn{2}{|c|}{ 3-D } & \multirow{2}{*}{$\begin{array}{l}\text { 3-D } \\
\text { Separated } \\
\text { Flow }\end{array}$} \\
\hline & & $\begin{array}{l}\text { Blunt } \\
\text { Body }\end{array}$ & $\begin{array}{l}\text { Lifting } \\
\text { Vehicle } \\
\end{array}$ & \\
\hline Parabolized Navier-Stokes & $\begin{array}{l}4 \mathrm{PG} \\
3 \mathrm{EC} \\
1 \mathrm{NEC}\end{array}$ & N.A. & $\begin{array}{l}3 \text { PG } \\
2 \text { EC } \\
1 \text { NEC }\end{array}$ & N.A. \\
\hline $\begin{array}{l}\text { Shock-Layer or Thin-Layer } \\
\text { Navier-Stokes }\end{array}$ & $\begin{array}{l}4 \text { PG } \\
4 \text { EC } \\
1 \text { NEC }\end{array}$ & $\begin{array}{l}3 \text { PG } \\
3 \text { EC } \\
1 \text { NEC }\end{array}$ & $\begin{array}{l}3 \text { PG } \\
3 \text { EC } \\
1 \text { NEC }\end{array}$ & 1 PG \\
\hline Navier-Stokes & $\begin{array}{l}3 \mathrm{PG} \\
3 \mathrm{EC} \\
1 \mathrm{NEC}\end{array}$ & $\begin{array}{l}3 \mathrm{PG} \\
3 \mathrm{EC} \\
1 \mathrm{NEC}\end{array}$ & $\begin{array}{l}2 \text { PG } \\
2 \mathrm{EC} \\
1 \mathrm{NEC}\end{array}$ & $\begin{array}{l}1 \mathrm{PG} \\
1 \mathrm{EC} \\
1 \mathrm{NEC}\end{array}$ \\
\hline Particulate Monte Carlo & 3 NEC & $1 \mathrm{NEC}$ & 1 NEC & $1 \mathrm{NEC}$ \\
\hline
\end{tabular}

KEY:

1. Evolving enabling technology

2. Understanding capabilities and limitations

3. Beginning to apply

4. Learning to use effectively

5. Mature capability

$P G=$ perfect gas

$E C=$ equilibrium air chemistry

$\mathrm{NEC}=$ non-equilibrium air chemistry

N.A. = not applicable

current status is judged to be at or approaching maturity; but, for complex 3D flows involving separated flow or inlet and airframe integration, for example, current codes are far from maturity.

Table 4, representative of hypersonic AOTV-type flight in the upper atmosphere, exhibits a number of blank boxes in the matrix. These signify that work in such areas is judged not yet to be in the first stage of research code development. In some of these cases codes are known to be under development, but have not yet reached grade 1 . This table, of course, represents CFD codes that are very much more complicated than those of Table 3 . They also require much more computation time because of the numerous chemical reactions that must be solved simultaneously with the equations of fluid motion. Thus, the efficiency of numerical algorithms is an important aspect of this type of code development. Table 4 indicates clearly that there is much research and development to be done to bring such CFD codes into a stage approaching

\section{I I I I}


maturity. Notably, the assessment made of the code development stage assumes that all necessary input data on reaction rates and so forth are available for the codes to use. As stated earlier this is not the case, and a separate and extensive level of research effort on molecular physics will be required to provide these data.

A significant point about the status of particulate flow simulation is not reflected in the entries of Table 4. Present Monte Carlo codes generally use human interaction to resize computational cells in accordance with the location in the flow field of high or low gas density. Consequently, the efficient exploitation of this type of flow simulation will require the development of some new automatic self-adaptive method for appropriately sizing computational cells.

\section{FINDINGS}

Hypersonic CFD spans a wide range in complexity dependent mainly on flight altitude: Air chemistry is in thermodynamic equilibrium within the lower atmosphere but is strongly in nonequilibrium in the upper atmosphere. The CFD codes for the lower atmosphere are only a modest extension of those for conventional aircraft, but the corresponding codes for the upper atmosphere are much more complex and in a more primative state of development due to numerous additional molecular physics processes that must be considered. Various hypersonic vehicles cover both the equilibrium and nonequilibrium regimes. The principal design problems of past vehicles (e.g., ICBM and Apollo) generally involved equilibrium flow. Potential NASA and DOD vehicles for the 1990 s and beyond involve higher altitudes and nonequilibrium flow. Other vehicles, such as the shuttle, involve some aspects of each regime.

The degree of accuracy of the conventional Navier-Stokes equations of motion becomes a major issue at altitudes well below the limiting altitude for continu um flow. These equations are demonstrably inaccurate in the upper atmosphere where the shock-wave thickness can be a substantial fraction of the shock detachment distance. Moreover, they are of uncertain accuracy at lower altitudes where the shock thickness is negligible and reaction rates are dominant.

The simulation of hypersonic flow fields undergoing nonequilibrium chemistry requires that vehicle scale be simulated as well as

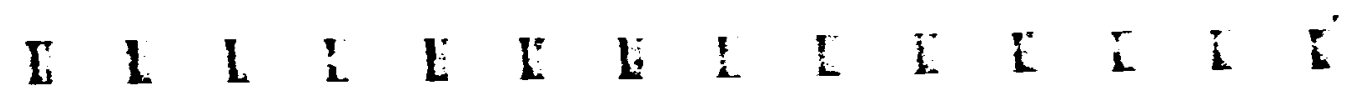


flight velocity and altitude. Because this is not possible in existing ground-based experimental facilities, the applications and the importance of CFD to hypersonic vehicle design is much greater than for conventional aircraft design. For flight in the upper atmosphere, CFD is expected to be the principal source of flow simulation information for vehicle design, and flight test is necessary for accurate validation of the computational codes.

A large amount of molecular physics data is needed as inputs for both continuum and particulate types of flow simulation. Data on many chemical reaction rates, excitation rates, gas-surface reactions, and transport properties are needed especially for the higher range of temperatures encountered.

The computational efficiency of numerical algorithms is of increased importance because of the long computing times involved when a large number of chemical reaction and species diffusion equations must be solved simultaneously with the equations of fluid motion.

Because of the much lower Reynolds numbers encountered in the upper atmosphere, the flows are mainly laminar and turbulence modeling is not anticipated to be a major technical problem. In the lower atmosphere, however, the location of transition and turbulence modeling is vital for hypersonic aircraft configurations.

\section{RECOMMENDATIONS}

The development of mature, fully realistic, and efficient computer flow simulations for the wide range in altitudes covered by advanced hypersonic vehicles will require considerable future progress in certain areas. Five particularly important areas warranting special attention are:

1. Molecular physics data base. Extensive physical data required as inputs for hypersonic CFD codes are needed on chemical reaction rates, excitation rates, gas-surface reactions, and transport properties at high temperature.

2. Continum equations of motion. Some inaccuracies in the conventional Navier-Stokes equations for hypersonic flow conditions need to be mended and new physics added to the continuum equations of motion if they are to integrate accurately through shock waves in the upper atmosphere.

3. Flight tests. Appropriately instrumented flight tests are necessary to validate computational codes for hypersonic flight in

I $\quad$ I L L I I

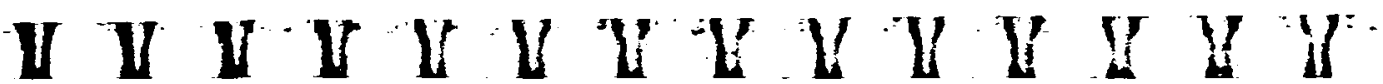


the upper atmosphere due to the inability of ground-based experimental facilities to simulate properly the chemically nonequilibrium flow.

4. Transition and turbulence. These problem areas can have a major impact on aerodynamic heating and payload weight.

5. Numerical methods. Algorithms of improved computational efficiency for both continuum and particulate flow simulations are needed to reduce the computer time required to simulate chemically nonequilibrium flows.

I 1 L I L L E L I I I 


\section{6 \\ Propulsion Applications of CFD}

\section{OVERVIEW}

Despite the complexities of the flows associated with propellers, helicopter rotors, aircraft gas turbines, ramjets and scramjets, and rockets, the pressing need for aerodynamic improvements has made the aggressive use of CFD inevitable. The history of propulsion is marked by pioneering efforts to harness the latest available computational capabilities, going back to the days when rooms full of engineering aides using mechanical desk calculators carried out component matching and compressible nozzle flow solutions. These beginnings set the stage for the fearless attack on even the most unyielding problems and for some remarkably successful achievements. Today, CFD is becoming a mainstay of the propulsion world.

The problems associated with propulsion differ from their external aerodynamic counterparts in three principal ways: (1) they usually involve complicated, even contorted, geometries and thus boundary condition constraints; (2) there is close coupling of flow elements involving 3-D flow, unsteady effects, and significant lengths of transitional boundary-layer flow (laminar to turbulent); and (3) there is a significant energy exchange from either chemical reactions or mechanical work.

While this list seems formidable and could have a daunting effect on the growth of CFD technology for propulsion systems, it has in fact done quite the opposite. Since this environment is so difficult to reproduce experimentally, CFD plays the added role of a basic data source, helping to identify the dominant physics

47
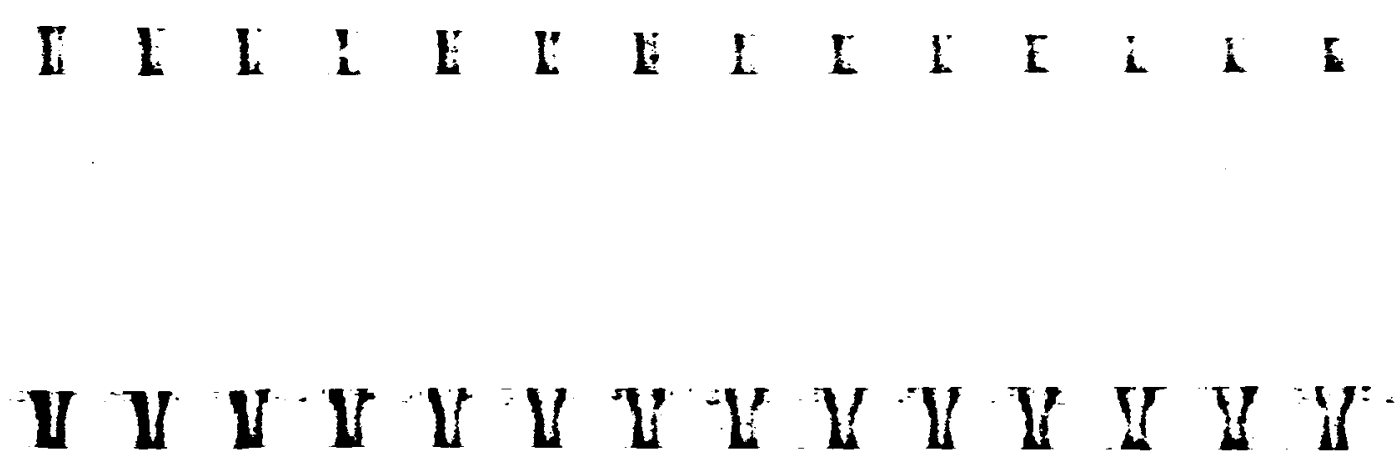
for a given situation so that analysts are better positioned to produce reliable design procedures. CFD is being employed as a breaching technology - one used to gain insight and control, and then penetration of the roadblocks facing advanced propulsion systems.

The propulsion CFD experience does share one important characteristic with the rest of the aerospace field, namely that there are many different ways in which CFD has made an impact. The three following sections illustrate the breadth and depth of the influence of CFD.

\section{Detailed Design of Components}

There are many engine parts with aerodynamic roles whose geometrical surfaces are directly determined by CFD. An outstanding example concerns the current method of designing the contours of propeller, compressor, and turbine airfoils in which each is carefully created for its specific usage by means of compressible flow and boundary-layer calculations. This precision approach has replaced the hit-and-miss application of standard airfoils drawn from extensive families of relatively simple airfoil shapes (e.g., multiple circular arc) which did not take advantage of modern understanding of aerodynamics.

Improvements through the use of CFD in airfoil contour design are significant because they reduce and control airfoil drag, the root cause of inefficiency, in a fundamental and sound way. Moreover, in the propulsion world a small increase in component efficiency has a remarkably large influence on fuel used or payload delivered over the life of the system. A more subtle but revealing indication of the significance of this change has been the appearance of such brand names as "controlled-diffusion" and "tailored" airfoils.

There are other engine components that benefit from the application of CFD. Some leading examples include: the internal passage design of inlets, transition ducts (between components), and nozzles; the geometry of combustors; the shaping of afterburner mixers; and the external design of nacelles, including inlets and boattails, taking into account the influence of adjacent surfaces.

The clear trend is toward a greater use of CFD. In particular, the extension of 2-D potential flow calculations to 3-D and from

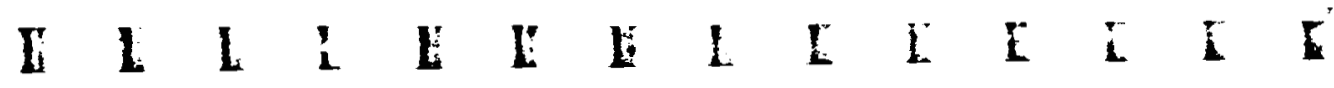


simple boundary-layer models to complex ones continues with the goal being to achieve improved performance on the first try.

\section{Development of Insight and Understanding}

An abundance of auxiliary tasks are regularly accomplished by CFD and provide a sound foundation for progress in aerodynamic design.

With close interaction between CFD and experimentation, advanced computer programs are used both to design an experiment and to predict the results so that the test is under control at all times. In some extreme cases, experimental data that had first been disregarded because it had no logical explanation was later reproduced by sophisticated CFD calculations. This not only exonerated the suspect facility, but also provided a clearer understanding of the underlying aerodynamic phenomena. In such cases CFD helped delineate the dominant physics of the phenomenon of interest.

The complementary interplay of CFD and experimentation is especially important in the modern world of tailored designs, where the goal is not to generate half-blind global correlations, but to prove the accuracy of a total design system by means of benchmark demonstrations in reliable facilities. Consider here, for example, the large number of combinations of expensive and timeconsuming cooled turbine airfoil configurations that would have to be tested in order to find the best for a given situation. Once confidence in CFD methods has been established, the search can be primarily carried out on paper. Moreover, the designer gains a clear understanding of why the choices have been made.

Even when available CFD tools are not extremely precise, they have often been used to aid designers in what might be called a semiquantitative fashion. The importance of the concept follows the well-known principle that if one can show an engineer where the flow goes and why (qualitatively) then he can usually figure out how to control it better. Examples that fit into this category are not to be taken lightly and include: the calculation of trends and trade-offs in cases where the principal need is to find the direction of change most likely to improve performance; the visualization of flows (e.g., streamlines, vector fields, vorticity distribution, and pressure, temperature or velocity profiles) to explain observed behavior and to provide a feel for the flow which can lead to

\section{I I I I I}


improvements; and the almost classical use of early CFD codes to explain the complex effect of airfoil shape on transonic flow and of freestream turbulence on boundary-layer transition.

\section{Possibility for Revolutionary Change}

The propulsion world is best described as evolutionary because progress is traditionally gradual and based upon the last successful design. Radical changes happen only occasionally, and are often the result of necessity, such as the need for ultra quiet and low fuel consumption commercial engines or the need for highly flexible fighter engines. Among the reasons for this apparent conservatism are the enormous costs and times associated with the demonstration of a new propulsion aerodynamic technique, especially when the realities of actual operation must be considered. The important point is that propulsion systems necessarily have closely coupled and complicated aerodynamic elements that are hard to understand independently - much less in interactive roles.

CFD is breaking this pattern with increasing frequency because it makes possible the accurate assessment of entirely new aerodynamic ideas. Several samples illustrate this trend.

In 1968 streamline curvature CFD methods were used to demonstrate the concept that controlled vortex (i.e., not free vortex) turbine stages can produce more power at high efficiency than had previously been thought possible. This idea was soon proven conclusively by experiment and adopted by the turbomachinery industry.

In 1971 many of the remaining barriers to the confident design of both cooled and high-power turbine airfoils were broken through the development of reliable boundary-layer and potential flow calculation techniques. These became the building blocks for many other advances including transonic and laminar turbine airfoil designs.

In 1975 advanced streamline curvature CFD methods were used to incorporate several simultaneous improvements (e.g., controlled diffusion, swept leading edges, and sloped inner wall) into an advanced, high-pressure ratio, transonic compressor stage design. The result, also demonstrated by experiment, was a truly remarkable fan that has had a favorable influence on all subsequent

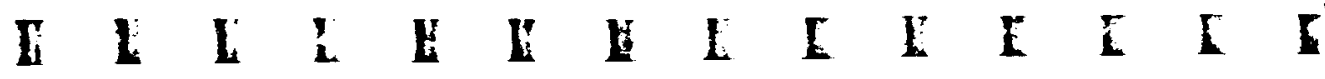


compressor and fan designs. In this case CFD clearly made possible the most important forward leap of compressor technology of the past generation.

In 1980 the need to squeeze maximum performance from the integrated powerplant, i.e., engine plus airframe, led to the harnessing of 3-D, compressible CFD codes for the entire design of transport aircraft nacelles. This approach led to substantial drag reductions with no adverse impact on the required operational characteristics of the nacelle, e.g., angle of attack stability, and windmilling and reverse thrust performance.

Very recently, in 1984, flow separation in the space shuttle main engine turnaround channels (which deliver the hydrogen from the high-pressure fuel turbine to the injectors) was eliminated by means of a CFD analysis requiring the Cray XMP computer. This improved performance by reducing pressure drops and increased life by making the outlet flow more uniform and steady. Special attention to graphic displays enabled maximum advantage to be taken of the results.

\section{CFD CAPABILITIES IN PROPULSION}

To assess CFD capabilities in propulsion an attempt is made in the following section to determine the current degree of CFD use, identify its strengths and shortfalls, and establish a reasonable basis for setting both short- and long-term directions. To this end, this vast subject heading is broken down into generic technology areas that represent the critical flow aspects of the propulsion system components. As shown in Table 5 the three major headings used here to categorize all propulsion system elements are: (1) stationary systems-covering all 2-D and 3-D inviscid and viscous flows; (2) rotating systems-focusing on all unsteady and cyclical flow elements; and (3) combustor systems-including combusting and noncombusting flow elements. These are then assessed according to their technical maturity. As listed in the vertical column of Table 5 these are: inviscid models (full-potential and Euler equations), classical boundary-layer approaches (transitional and turbulent), inviscid and viscous interaction approaches, using both integral and finite difference (or volume) techniques, and composite viscous approaches employing either the parabolized (sometimes referred to as reduced) or turbulence-averaged NavierStokes equation set.

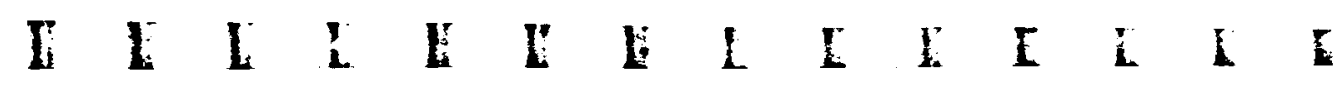


TABLE 5 Status of CFD for Propulsion Applications

\begin{tabular}{|c|c|c|c|c|c|c|c|c|}
\hline \multirow{2}{*}{$\begin{array}{l}\text { Flow Complexity } \\
\text { Governing Equations } \\
\end{array}$} & \multicolumn{3}{|c|}{ Stationary } & \multicolumn{3}{|c|}{ Rotating } & \multicolumn{2}{|c|}{ Combustors } \\
\hline & 2-D & 3-D & Unsteady & 2-D & 3-D & Unsteady & 2-D & 3-D \\
\hline INVISCID & & & & & & & & \\
\hline Full Potential & 5.0 & 4.0 & & 4.5 & 4.0 & 0.5 & & \\
\hline Euler & 4.5 & 3.5 & 3.0 & 4.0 & 2.5 & 1.5 & & \\
\hline VISCOUS & & & & & & & & \\
\hline $\begin{array}{l}\text { Transitional/ } \\
\text { Turbulent } \\
\text { Boundary Layer }\end{array}$ & 4.5 & 3.0 & 1.5 & 4.0 & 2.5 & 1.0 & 2.5 & 0.5 \\
\hline $\begin{array}{l}\text { Parabolized } \\
\text { Navier-Stokes }\end{array}$ & 4.5 & 3.5 & 1.0 & 4.0 & 2.5 & 0.5 & 4.5 & 3.5 \\
\hline $\begin{array}{l}\text { Viscous-Inviscid } \\
\text { Interaction }\end{array}$ & 3.5 & 1.0 & 0.5 & 2.5 & 0.5 & & & \\
\hline $\begin{array}{l}\text { Turbulence } \\
\text { Averaged } \\
\text { Navier-S tokes }\end{array}$ & 3.5 & 2.5 & 1.5 & 2.5 & 1.0 & 0.5 & 2.5 & 2.0 \\
\hline
\end{tabular}

KEY:

1. Evolving enabling technology

2. Understanding capabilities and limitations

3. Beginning to apply

4. Learning to use effectively

5. Mature capability

\section{Stationary Systems}

The stationary elements of a propulsion system include inlets, nacelles, diffusers, and nozzles together with the transfer ducts connecting the other components of the system. In applying CFD to these elements the analyst first needs to understand the structure of the flow including the location and magnitude of secondary flows and separated regions. Next the local values of the wall shear stress and surface heat transfer are needed. For supersonic flows the location and structure of the shock wave-boundary layer and bleed interactions are important. Finally, the analyst needs to be able to predict the loss of total pressure in the system. All of these concerns dictate that some form of 3-D viscous computation be employed, the lower two-thirds of the stationary 3-D column in Table 5. On the other hand, the trend toward more complex geometries with nonuniform upstream flow conditions for these systems has meant that large numbers of grid points, well in excess of $10^{5}$ in recent applications, are required to resolve the flow structure. As a result, even with the advent of the modern supercomputer, the use of the full turbulence-averaged Navier-Stokes

\section{$\begin{array}{llllllllllllll} & \mathbf{L} & \mathbf{L} & \mathbf{I} & \mathbf{E} & \mathbf{L} & \mathbf{E} & \mathbf{L} & \mathbf{L} & \mathbf{L} & \mathbf{I} & \mathbf{i} & \mathbf{I} & \mathbf{E}\end{array}$}


equations is generally considered too expensive for practical applications. Instead, extensive use is now made of parabolized (or reduced) Navier-Stokes equations with spatial marching procedures for both subsonic and supersonic flows. The primary exception to this occurs in the analysis of an inlet at angle of attack where 3-D potential and Euler codes are in use. Since the pressure variations are quite large for this problem, inviscid approaches provide much useful information.

There are many aspects of flows in inlets, diffusers, and nozzles for which the status of current CFD tools may be considered inadequate. Examples of these include 3-D shock and boundarylayer interactions, local separation regions, surface heat transfer, injected coolant flows, and cases with large amounts of bleed. The status of unsteady viscous analyses for these components is particularly weak (see Table 5). In addition, the nature of flows in complex geometries with rapidly varying cross sections is not wellunderstood. Finally, the ability to predict the loss in total pressure for these systems is not yet adequate. In general, whether the fault lies with insufficient numerical resolution or with inadequate turbulence models is not known. However, in all the above cases it can be stated that the lack of a data base of sufficient detail to answer the numerics versus turbulence question is a major factor stifling the application of CFD.

\section{Rotating Systems}

The rotating elements of propulsion systems include fans, compressors, turbines, propellers, and helicopter rotors. As with the stationary elements the CFD analyst is interested in flow structure, including secondary flows and separated regions, values of wall shear stress and surface heat transfer, the nature of shock and boundary-layer interactions, and the prediction of losses in total pressure. There are, however, two significant differences from the stationary case. First, these flows are inherently unsteady, a fact which is just now beginning to attract attention in the CFD community. Second, the pressure variations are even larger than in the stationary cases and as a result inviscid treatments again can provide much useful information.

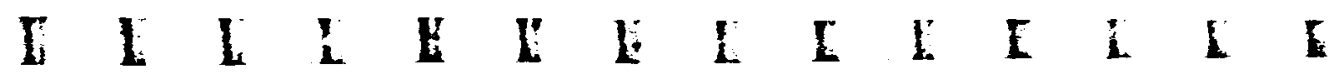


Most analyses treat a hypothetical steady flow obtained by assuming axisymmetric conditions upstream of a single turbomachinery passage. There is extensive use of inviscid codes, 2-D potential and 3-D Euler, in the design and analysis of rotating components. Two-dimensional boundary-layer codes are commonly used to estimate losses and surface heat transfer. Two-dimensional Navier-Stokes codes are becoming available but are not yet in frequent use. Three-dimensional Navier-Stokes codes are under development but most of those in use are proprietary.

In short, there is extensive development activity in most of the boxes in the rotating 2-D and 3-D columns of Table 5. There are also a number of efforts aimed at computing the 2-D unsteady viscous rotor and stator interaction problem, and there is one effort under way to model the $3-\mathrm{D}$ viscous averaged flow in multistage turbomachinery. As shown in Table 5 the level of development for unsteady turbomachinery codes is rudimentary. The true multistage problem is unsteady, nonperiodic, and well beyond the capacity of even the next generation of supercomputers. Hence, some approximate approach must be found for this important class of problems.

As is the case with stationary components, current CFD tools are inadequate to analyze many aspects of rotating machinery flows, e.g., the structure of secondary flows and separation regions, surface heat transfer with and without injected coolant, and the predicton of total pressure loss and aerodynamic loads due to flutter or gust response on blades, rotors, and propellers. This is complicated by low Reynolds numbers, under $5 \times 10^{5}$, and high freestream unsteadiness, 10 to 20 percent. This results in an inability to predict wall shear stress and surface heat transfer on turbine blades where boundary-layer transition may occupy $\mathbf{4 0}$ percent or more of the blade chord. The general question of insufficient numerical resolution versus inadequate turbulence models is even more difficult to answer in the rotating than stationary case because the CFD problem is considerably more difficult. However, it can be stated that the lack of a data base of sufficient detail to resolve the numerics versus turbulence issue for rotating machinery is an even larger roadblock for CFD development than it is in stationary components. In the future it is expected that much of the emphasis will switch to time-resolved measurements of these flows as the CFD community mounts a significant effort to address unsteady turbomachinery computation.

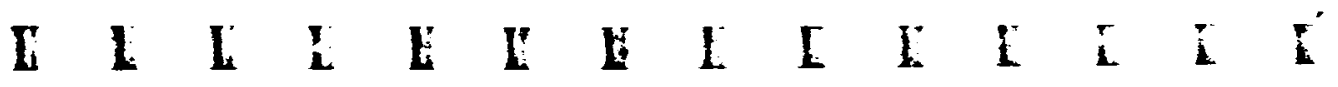




\section{Combustors}

The focus here is on CFD as it applies to the aero/thermodynamic elements of the entire combustor, including both combusting and noncombusting elements of such flows. It is useful to think in terms of four major aspects of combustors: (1) oxidizer/fuel intake and management which can involve significant regions of axially reversed flow; (2) oxidizer/fuel mixing and combustion involving multiphase and multispecies tracking, heat addition, and surface heating; (3) exhaust flow management with large secondary flows, dilution, and cooling; and (4) massive coupling and interaction of the first three. Within these four areas there are five generic computational flow problems that can be identified and prioritized according to ascending order of difficulty: (1) largescale secondary flow mixing; (2) reverse flow; (3) heat transfer, both convective and radiative; (4) combustion, including phase and species change and stability; and (5) flow coupling in complicated geometries. With this in mind, one notes that in Table 5 , the only areas of significant activity are those involving viscous effects; boundary layers for surface heating; and parabolized and full turbulence-averaged Navier-Stokes for the remainder of this complicated environment.

With regard to secondary flow mixing, this type of flow problem is encountered at two possible sites in a combustion process, at the head end where rapid mixing of the fuel and oxidizer is desired or at the aft end where dilution of the hot exhaust gases is required to avoid destroying downstream components, such as a turbine. The flow involves 3-D free shear layer turbulent mixing and possibly swirl. Additionally, liquid fuel droplet spray must be introduced and tracked through its phase-change just prior to the combustion process. Current approaches generally focus on use of the time-averaged turbulent version of the Navier-Stokes equations or a parabolized version of the same if reverse flow is not encountered. In either case, as indicated in Table 5, the overall problem is fairly well in hand numerically with three principal frontiers remaining, those of reducing difficulties related to numerical diffusion errors, improving fuel spray tracking models, and establishing a credible basis for modeling of 3-D turbulent mixing. Since turbulence modeling is discussed in more detail in Chapter 7 it will not be pursued further here except to note its critical importance to this flow arena.

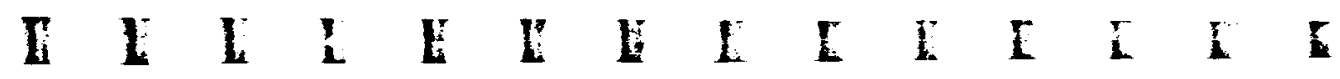

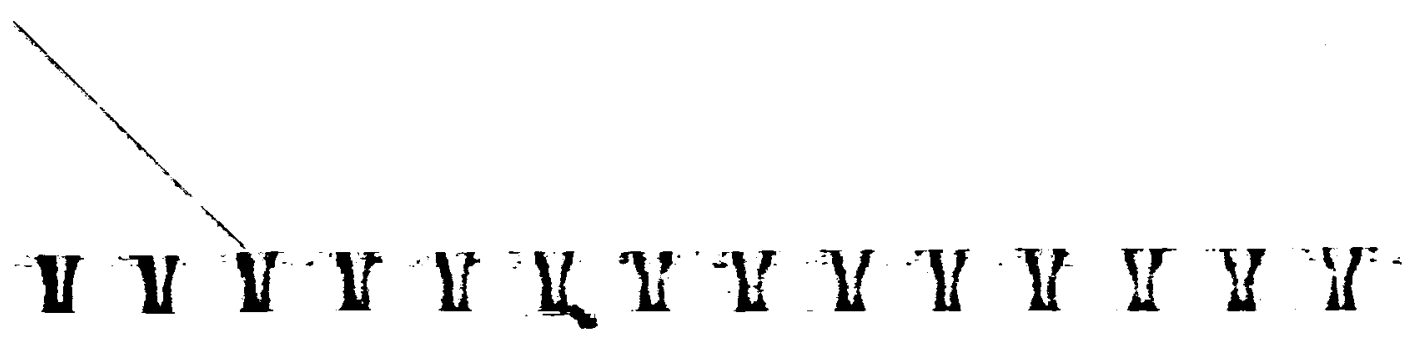


Numerical diffusion difficulties force the use of relatively fine meshes to resolve the thin viscous mixing regions embedded in otherwise large-scale vorticity-dominated flows. While this aspect of the problem is receiving considerable attention, there is still a need for a breakthrough in this area to reduce the computer intensive aspect of the flow mixing problem. The fuel tracking problem involves tedious accounting of large ensembles of spray and species elements that again cause considerable drain on computer resources. This is especially critical to the field of scramjet combustion where the physics of the fuel droplet tracking in a supersonic stream represents the key element of the combustion process. Underlying this is the lack of a good analytical model representing the dominant physics of liquid spray formation.

With regard to recirculating flow it is safe to say that nearly all aerospace combustion processes encounter some form of flow reversal in the principal flow direction. In some instances it is desirable as a flame ignition and stability mechanism (e.g., combustordomes, augmentors, and flame holders) while in others it is a necessary evil (e.g., dump diffusors or scramjet side walls). In either case, it usually is of relatively large scale and plays a significant role in the device's effectiveness-thus making its prediction of primary importance.

The general approach used to attack this problem employs algorithms to solve the full turbulence-averaged Navier-Stokes equations although recent works based on interaction concepts have indicated that some simplification of the diffusion process is possible. There has been good progress in the solution of such flows at the 2-D level with pioneering work being focused on the 3-D problem. Here the two principal difficulties appear to be elimination of false (numerical) diffusion and modeling of real turbulent diffusion. This problem is especially acute and important because it competes with the real physics in the process that controls the combustor and combustion process. More research focused on the numerical representation of 3-D thin viscous shear layers between regions of forward and reverse rotational flows is needed to avoid the need for massive computer resources in representing the critical physics.

With regard to heat transfer it is obvious that combustor durability is critically dependent on the ability to assess heat loads due to convection and radiation to the walls. Current CFD technology is unable to help much in representing the convection

\section{$\begin{array}{llllllllllllll}\text { I } & \mathbf{L} & \mathbf{L} & \mathbf{L} & \mathbf{E} & \mathbf{E} & \mathbf{E} & \mathbf{L} & \text { L } & \text { I } & \text { E } & \text { I } & \text { I } & \text { I }\end{array}$}


heat load due to the extremely complicated nature of the surface boundary layers (e.g., very thin, 3-D, and film cooled). Accurate prediction of the radiative heat load (which can equal or even dominate the convective load) is limited due to the inability to solve very sophisticated radiation models efficiently and the lack of a simpler representation of the processes involved.

The numerical representation of the combustion process in aerospace application is a frontier problem. Although some progress is being made in the use of simplified (equilibrium chemistry) approaches, the field is still in the embryonic stage. Although very significant and promising efforts are presently ongoing, major challenges remain in the modeling of fuel phase change, finite rate chemistry, heat addition, turbulence, and large-scale instability representation. The problem area is ripe with opportunity because there exists both the need and use for fundamental work at all levels: delineating the dominant physics through benchmark experimentation; identifying new analytical models and their partial differential equation representation; and developing new solution methodologies employing novel computational techniques.

The final area of concern is that of flow coupling. Nearly all combustors encounter very close coupling of multiple flow element interactions that are difficult to represent numerically. A vivid example is that encountered in a gas turbine where the inlet air is split into multiple 3-D passages and introduced into the combustion process through multiple ports possibly with swirl and certainly at large angles to the axial direction. The interaction of these multiple elements still represents a major challenge to numerical grid generation and boundary condition representation for the problem, and are only now beginning to be faced at the 2-D level.

\section{FINDINGS}

Computational fiuid dynamics is used extensively throughout the propulsion community principally because of a lack of alternatives for achieving insight into the basic controlling mechanisms of the complicated, highly coupled interacting aspects of propulsion fluid dynamics. It has proven a valuable design analysis tool in a wide variety of situations, often more than expected. Use of CFD is rapidly spreading and growing largely due to its ability to provide detailed diagnostic data and to address more complex flows. One

I $\quad$ I L L I 
of the principal challenges now being faced is that of providing a way for this powerful but costly and time consuming capability to contribute directly to the formal design for complicated, strongly coupled propulsion system components.

There has been a rapid increase of interest in advanced engines to propel hypersonic and transatmospheric vehicles. The flow conditions found in these machines are so demanding that they cannot be reproduced in existing facilities. Consequently, the design and evaluation of these enormously powerful engines can only be done using CFD. Although existing codes have inadequacies in turbulence and chemistry modeling, they offer the only available capability and will carry an unusually large share of the design burden. Reliance upon thinly substantiated data bases and design tools places critical hardware programs at undesirable risk.

In general, propulsion CFD is in a relatively mature state for 2 $D$ potential flows with its capability dropping off systematically with the level of complexity. This can be better summarized in terms of the three major elements of propulsion devices: stationary systems, rotating systems, and combustors. For stationary systems CFD technology is in a relatively mature state for 2-D configurations, with a rapid drop-off in capability for 3-D or unsteady flows. Rotating systems capability naturally lags its stationary counterpart at all levels-most seriously in the area of viscous flow modeling. Combustors, themselves inherently complicated, show the lowest level of CFD development.

The strong aerodynamic coupling of the major components of propulsion systems is another important issue. Examples of this include the influence of inlet distortion on the performance, stability, noise, and durability of the fan, the impact of burner exit temperature profiles on turbine life, and stagnation (nonrecoverable) stall. With the advent of larger capacity parallel processing computers, the time is rapidly approaching when serious consideration can be given to modeling interacting components using these unique computers and yet-to-be-perfected algorithms.

The final area of overall concern involves experimental data bases. There is a definite need for benchmark experiments to assist and guide the development of codes especially with respect to turbulence modeling. Note that benchmark experiments are generally those that provide detailed data bases against which codes can be calibrated. Additionally, there is a need for diagnostic experimental studies that can provide the sort of detailed data that allow

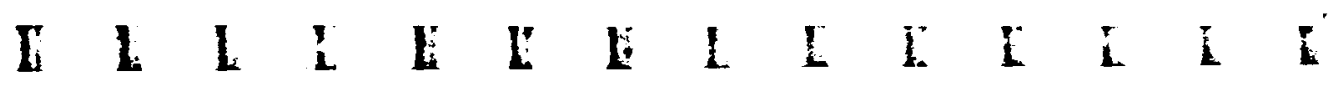


identification of the dominant physics controlling certain mechanisms and critically needed insight for establishing appropriate levels of analytical modeling.

\section{Stationary Systems}

For stationary systems, Table 5 provides a clear picture of the situation-for 2-D flow the computational technologies are well in hand. Additionally, as a third dimension is invoked (be it space or time), capability drops off dramatically as one encounters the complications of viscous effects to resolve near wall effects (e.g., skin friction, heating, shock-wave boundary-layer losses, and bleed flows). Complicated 3-D geometries require very large grids $\left(>10^{5}\right)$ and codes that are still too expensive to be of utility to any except a small segment of the potential user community. Additionally, turbulence modeling is rapidly becoming a critical issue because we are reaching the point where it is the final key factor in determining the payoffs in terms of durability and performance. This is one of the chief reasons why we must maintain an extensive program of benchmark and diagnostic experimentation for calibration of codes or turbulence model improvement. As a final but significant issue, there is a growing concern about numerical errors due to artificial viscosity, numerical diffusion, and other factors. A large number, perhaps the majority, of codes today contain "knobs" that set levels of these artificial effects in admittedly ad hoc fashion, thus limiting their credibility. This is especially important for hypersonic flows where a very high level of uncertainty exists.

\section{Rotating Systems}

With regard to rotating systems, Table 5 shows trends somewhat similar to that for stationary systems because this technology is following closely and building from its stationary counterparts. The major areas of concern are accurate modeling of transitional flow effects and unsteady gas dynamics. Transition modeling is especially important for propulsion systems where relatively low Reynolds numbers are encountered and the length of transition is a significant fraction of the component's length (greater than 10 percent of chord on turbine blades) or there are large freestream vortical disturbances feeding the transition process. As in the
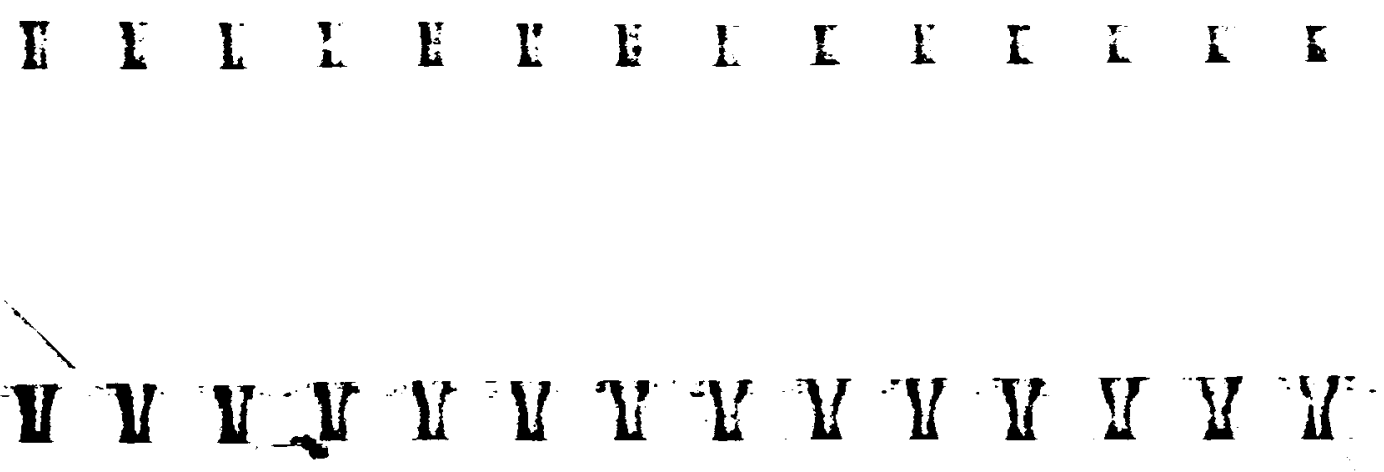
stationary system case, benchmark diagnostic experimentation is presently required to relieve this uncertainty. With regard to unsteady gas dynamics there is a persistent need for advances in the analytical and computational modeling of flutter type gas dynamics, rotor and stator interactions, and noise. Advanced blade and propeller concepts are being paced by the inability to predict accurately the unsteady fluid dynamics encountered in catastrophic aeroelastic events.

\section{Combustors}

For combustor systems, the story depicted in Table 5 needs some explanation. In general, these flows are so complicated that today only parabolized or turbulence-averaged Navier-Stokes approaches are fruitful. Some work on boundary layers is meaningful but only to the degree it helps resolve convective heating and viscous loss details near the combustor walls. Surprisingly, one finds in Table 5 that the capability levels for combustors in a given area are quite close and even ahead of the respective elements for stationary or rotating systems. This is not considered contradictory but rather reflects the relative nature of the grading system. Very rapid and encouraging progress is being made in this area, especially with regard to the numerical representation of inherently complicated geometries. The principal issues faced today are those of modeling mixing due to either turbulence, large scale secondary flows (steady or unsteady), or false diffusion. This, of course, is critical for it directly controls fuel distribution, phase change, heat addition, chemical kinetics, and other factors. The area of surface heat transfer (convection and radiation) is only now being addressed and will prove to be a pacing issue for CFD in the near future.

\section{Aerodynamic Coupling}

There is one remaining important issue to be considered-the strong aerodynamic coupling of the major components of propulsion systems. Important examples of this have included the influence of inlet distortion on the performance, stability, noise, and durability of the fan and the impact of burner exit temperature profiles on turbine life. The outstanding case of this type is stagnation (or nonrecoverable) stall, which results from the interaction

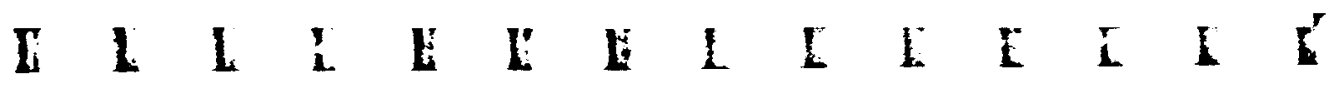


of at least the compressor, burner, and turbine, and causes the engine to lose thrust without either warning or a simple method to recover. With the advent of larger capacity parallel processing computers, the time is rapidly approaching when serious consideration can be given to modeling interacting components using these unique computers and yet-to-be-perfected algorithms.

\section{RECOMMENDATIONS}

As delineated in the above discussions there are several clearly defined areas that require further work in propulsion CFD:

Turbulence and transition modeling for use in the turbulenceaveraged Navier-Stokes equation modeling of free shear and boundary layers is a critical issue for propulsion CFD. While significant and rapid progress is being made in most other related areas, little work is being focused on this item. The turbulence model is rapidly becoming the technology limit. Work should be focused in two areas: (1) generation of benchmark diagnostic experimental data bases at a time-resolved level, and (2) development of new turbulence models (time-averaged and time-resolved) focusing on freestream disturbance effects (e.g., unsteadiness, turbulence, and vorticity) and transition prediction.

Elimination of numerical diffusion, artificial dissipation, and smoothing procedures in CFD codes will be critical to enhancing their credibility and utility. Most large codes today are saddled with "knobs" that are empirically set to obtain the best fits and convergence. The inability to transform properly the integral or differential versions of the conservation laws for vortical and viscous flows into discrete linear algebraic equations is the issue here. Focus should be given to developing "smart" finite difference schemes with good fluid dynamics for the chosen algorithm.

Analytical and computational modeling of aeroelastic loadings on blades and propellers continues as a frontier issue. Work should be encouraged in this unique, tedious, nonlinear environment with the goal of providing a reliable predictive tool to the structural analysis community.

New techniques for predicting viscous wall effects and processes (e.g., skin friction, heat transfer, separation, and bleed) with reduced grid would greatly reduce computer power needs. Accurate prediction of such parameters is of critical concern to the performance and durability of propulsion systems. Yet the high "grid

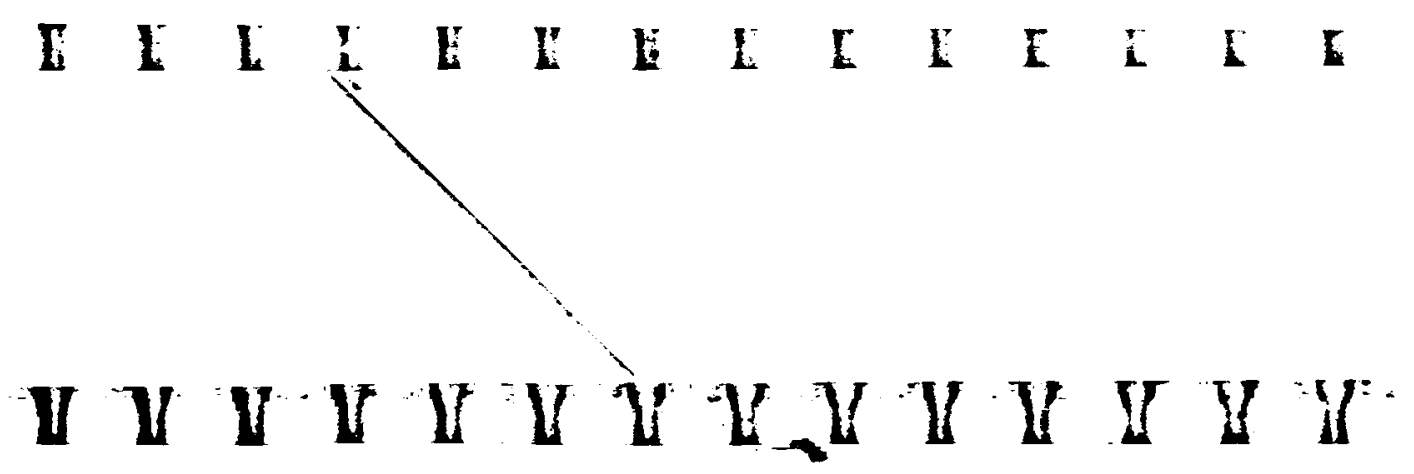


tax" required to calculate these accurately is limiting propulsion CFD's utility. Therefore, attention should be focused on the development of new schemes for resolving the high gradient regions of turbulent and high Mach number fluid and thermal boundarylayer regions.

Large-scale mixing phenomena as induced by axial vorticity should be studied further both analytically and experimentally. This technology would have a direct and significant payoff to the combustor and observables community. The principal issue here is resolution of the dominant physics and development of the analytical and numerical representation of those data.

Three-dimensional unsteady fluid dynamics is at the heart of numerous elements of multistage turbomachinery. Computational techniques are needed to capture the multiscale elements of such flows, e.g., unsteady loads, heating, skin friction, and noise spectra. Additionally, time-resolved data are needed to sort out the important physics and provide a data base for modeling.

Modeling of the interactive coupling of large aerodynamic components as it occurs in propulsion systems is a future issue for $C F D$. Representative examples are: airframe and engine integration, multistage compressors and turbines, inlet and fan interactions, and counterrotating prop-fans. For such environments it seems reasonable to expect the use of parallel processing computer architectures to be of key importance. There is a need for a small number of highly focused pathfinding programs aimed at establishing algorithms capable of handling this degree of sophistication.

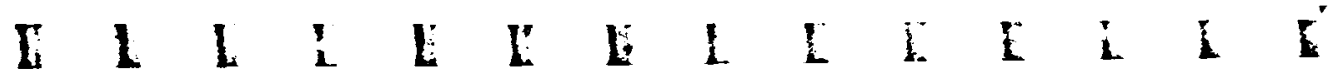




\section{7 \\ Turbulence in CFD}

\section{OVERVIEW}

Turbulence greatly enhances the transport of energy, momentum, and mass across a given region and the rate of mixing and chemical reaction within the region. This occurs by a complex process of time-dependent, 3-D motions in which vortex stretching and distortion produce small scales of motion responsible for molecular mixing, and vortex amalgamation produces the large scales of turbulence responsible for turbulent transport. This vorticity arises from viscous effects at solid surfaces and from density gradients.

Turbulence contains a broad spectrum of scales. The largescale features of turbulent flows are strongly dependent on the flow geometry and environment, e.g., rotation. Small-scale turbulence, which adjusts more rapidly to changes, tends to be more isotropic but also reflects the environment in which it is embedded (the larger-scale turbulent motions). The large-scale motions are responsible for turbulent transport, and the small-scale motions provide intimate molecular mixing.

These effects are included in CFD predictions in two general ways. With a turbulence "model" one tries to represent the average effects of these complex processes on the mean flow; this is very difficult to do in general because of the strong sensitivity of the turbulence to flow conditions. In turbulence "simulation" one includes the large-scale, time-dependent motions in the computation, perhaps using a model for the effects of the smaller scales;

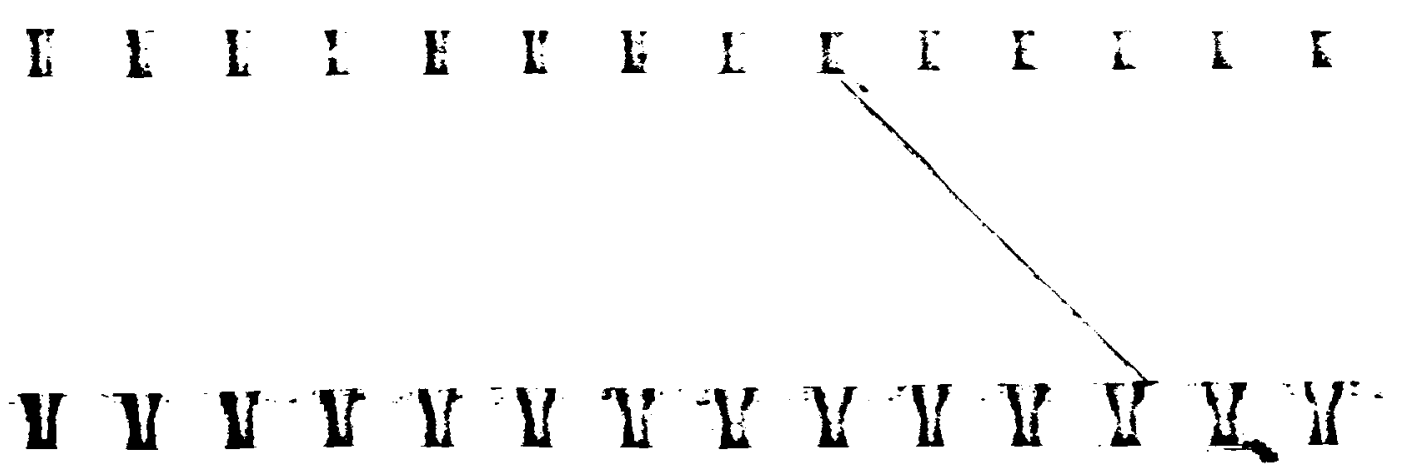


this is difficult because of the increased resolution required and the need for adequate prescriptions of turbulent boundary conditions.

Whether turbulence is important in a flow computation depends upon the purpose of the computation. Turbulence is becoming increasingly important as CFD is applied to more complex flows. For example, in external aerodynamics with attached flow the engineer or designer often can get what he needs without consideration of turbulence; however, turbulence must be considered in analysis of gas turbine engines and high-performance aircraft.

In many engineering problems turbulence is confined to thin shear layers, either in a boundary layer along a surface or in a free shear layer or wake. If the layer is thin, then the steady pressure field is affected only slightly by the turbulence, and an accurate calculation of the mean surface pressure (e.g., lift and form drag) can be made with the simplest of turbulence models. However, as one seeks to refine the accuracy of skin friction predictions, or to predict heat transfer, then good turbulence modeling becomes essential. Or, if one needs to know details of the time-dependent pressure field, say for load or sensor analysis or to determine the radiated sound, a very detailed consideration of the turbulence is essential.

Transition from laminar to turbulent flow is important in external aerodynamics at high altitudes and in gas turbine engines under all operating conditions, and results in substantial increases in skin friction and heat transfer over the levels for laminar flow. The ability to predict the location of transition and its effects is essential for good design of such systems.

Flow separation localized by a leading edge will cause the discharge of vorticity from the boundary layer into the external flow. Since the rate of spanwise mean vorticity flux in a boundary layer, for a good approximation, is dependent only on the freestream velocity and not on the structure of the boundary layer, inviscid (Euler) computational methods that put this vorticity into the flow and convect it correctly can give surprisingly good predictions for the global motions and pressure fields, both steady and unsteady. However, if the separation point is not localized by a sharp edge, but rather by the interaction of the boundary layer with the external flow, then a good turbulence model is essential for an accurate prediction of even the coarsest aspects of the flow, such as the pressure field. This includes cases where the boundary layer separation is induced by a shock wave, the shedding

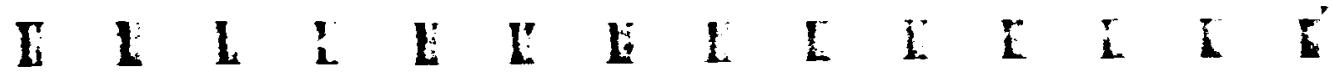


of wing-tip vortices, separation on fuselages, and separations in turbomachinery that affect blade heat transfer and system performance.

Separated flows often possess large-scale unsteadiness. If the computation is to represent the average flow, the turbulence model must include the effects of this unsteadiness. Or, if the computation will be time-dependent, and will attempt to resolve the large-scale unsteadiness, then the turbulence model must include the effects of the unresolved turbulence scales. If the unsteadiness is largely 2-D, a 2-D time-dependent computation might be used, but a model for the effects of 3-D turbulence structures must be included. These structures and the transport that they produce are strongly influenced by such effects as flow curvature and unsteadiness and these effects must be included in the turbulence model if an accurate description of the unsteady 2-D flow is to be obtained. Even if the computation is 3-D and time-dependent, with the goal of resolving all of the large-scale turbulence, a model for small-scale turbulence will still be required.

In turbulent flows with chemistry, detailed turbulence modeling is essential for accurate predictions of all parameters affected by the flow composition. In this case the thinness of the turbulent layer offers no escape from the need for detailed consideration of the turbulence and the need for a turbulence model. Since molecular mixing takes place on the smallest scales, accurate modeling of the small-scale turbulence is essential for accurate combustion predictions, even if the large-scale turbulence is resolved in the computation.

\section{TURBULENCE-AVERAGED NAVIER-STOKES EQUATIONS}

The Navier-Stokes equations (i.e., the exact equations of momentum, energy, mass, and species conservation in general timedependent, 3-D form) are assumed to describe the flow in full detail, including viscous flow phenomena and diffusive energy and species transport. Extensions of these equations may be necessary for nonequilibrium low-density hypersonic flows as discussed in Chapter 5.

Direct numerical solutions of these equations, accurate to all temporal and spatial scales of motion, are now practical only for the simplest of turbulent flows at relatively low Reynolds numbers.

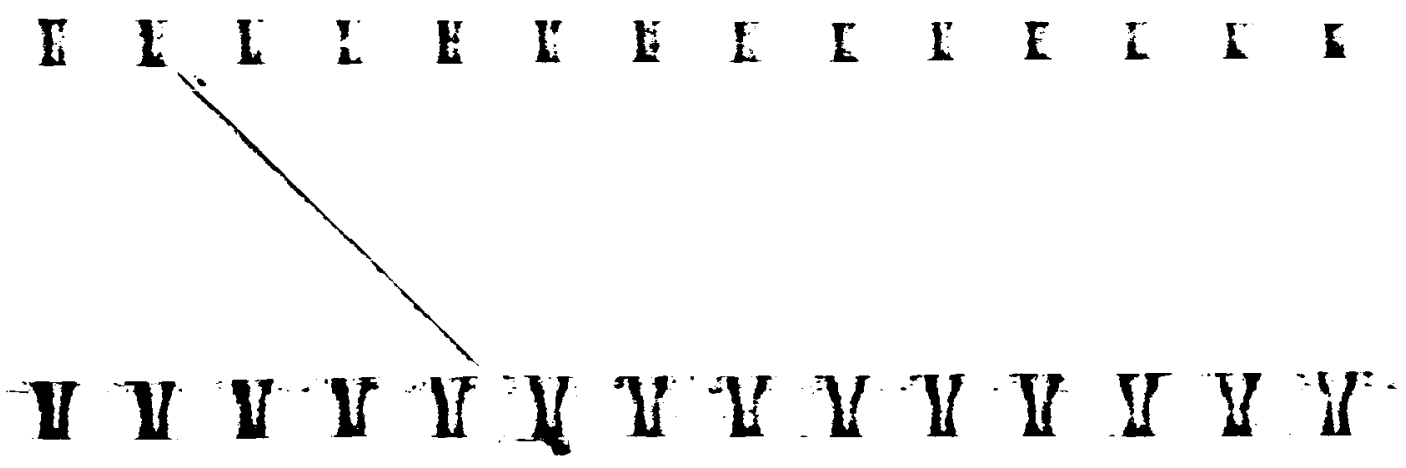


Such simulations are of great potential for use in evaluating and developing turbulence models, and this exploitation of CFD is in its infancy. Direct simulation of practical engineering flows at moderate and high Reynolds numbers is impossible now or in the foreseeable future. Large eddy simulations (LES) will also be of limited design utility for the remainder of this century.

Engineering calculations must instead be based on what we shall term the turbulence-averaged Navier-Stokes (TANS) equations. Practically all CFD calculations of the type reported to be Navier-Stokes are in fact based on one of two forms of turbulence averaging; most computations are based on the averaging procedure developed in the last century by Osborne Reynolds and are more properly termed "Reynolds-averaged Navier-Stokes," and most compressible computations employ Favre (mass-weighted) averaging, and would be more properly termed "Favre-averaged Navier-Stokes." LES are based on a different type of turbulence averaging, in which the averages are over the unresolved scales of motion. Other averaging methods, perhaps based on the orderedlarge-scale structures known to dominate turbulent flows, are expected to be used in the near future. This section refers to the Navier-Stokes equations, averaged by some appropriate method, as the TANS equations.

\section{CAPABILITIES OF TURBULENCE MODELS AND SIMULATION}

In many engineering problems the dependent variables of the TANS equations are all that is of interest. These include the mean velocity field, mean pressure field, and other mean properties. The TANS equations contain turbulent transport terms (e.g., Reynolds stresses and turbulent heat fluxes) for which models must be provided. In the usual formulations these involve statistical correlations between fluctuating components of quantities measured at the same point in space-time. In some cases other turbulence parameters are desired as computational output (for example fluctuation amplitudes or correlation lengths).

The job of the turbulence model is to provide an accurate representation of the connections between the turbulence quantities needed in the TANS equations and other turbulence parameters

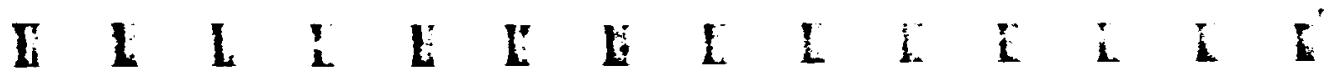


of interest. This prescription usually includes a combination of algebraic and evolution equations connecting the turbulence parameters with the TANS dependent variables. Since only one-point statistical quantities are required in the usual TANS equations, most turbulence models deal only with one-point turbulence parameters.

Ideally one would develop models in a hierarchical manner, with simpler models being derived from more complex models by simplifying approximations and the more complex models themselves being consistent with (and perhaps calibrated by) numerical simulations of turbulence. This is not the case, but it is the line of development that should be followed in the future.

For purposes of this discussion, turbulence models and simulations are divided into seven basic categories:

1. Local algebraic models

2. Scale-evolution models

3. Transport-evolution models

4. Probability density functions (pdf) models

5. Spectral interaction models

6. Turbulence simulations

7. Emerging ideas

The review of the status of each of these activities is based on a combination of the knowledge of the committee members in this area, the results from the surveys described in Appendixes $B$ and $C$, and discussions with leading researchers and CFD code developers.

\section{Local Algebraic Models}

These models relate the turbulent stresses and transports to local features of the mean flow, usually through a recipe for a mixing length. The survey revealed that there are many CFD codes incorporating models of this type. The Baldwin-Lomax model is the most common, primarily because of its ease of implementation, though other models in this class can be more accurate. This model was developed by experts in CFD, who for the most part did not have deep knowledge of turbulence. As a consequence its main virtues are computational utility.

Models of this type work reasonably well in the simple flows for which they have been tuned. They are more than adequate for

\section{I $\quad$ I L L}


problems in which turbulent transport is not important. Because they, in essence, assume that the turbulence has relaxed to equilibrium with the local mean conditions, they do not make any explicit consideration of the history of the turbulence. Consequently, they do not work well where the mean conditions change abruptly or whenever a significant region of the flow is affected by turbulent transport.

There is little new development in models of this type because physically motivated models with more structure are proving to be better tools for extending turbulence modeling to more difficult situations.

\section{Scale Evolution Models}

These models relate the turbulent transport to a combination of local mean field features and a few (1-3) scalar parameters of the turbulence. Partial differential equations for the evolution of scalar turbulence parameters are solved along with the mean momentum, energy, and mass conservation equations, which are coupled to the turbulence parameters through local algebraic stress tensor models. Thus, these models take explicit account of the history of the turbulence scales but assume that the nondimensional turbulence structure is in equilibrium with the local mean field and turbulence parameters.

The evolution equations are based on exact equations derived from the Navier-Stokes equations, with models for the unresolved statistical quantities. Usually one parameter is a velocity scale and the other carries the information on length scales; this avoids the need to prescribe the mixing length by an algebraic recipe. The k-epsilon 2-equation turbulence models are of this type. The committee's survey showed that a surprising number of CFD codes are capable of including such models.

These models generally offer a good first-order estimate of the characteristics and physics of simple separated flows or flows with gradual changes in boundary conditions, but can be less accurate than finely tuned algebraic models in some cases. Current models fail in flows with strong rotation, density gradients, energy release, and so forth.

The algebraic tensorial structure model is a critical element of such models. Many of these models use an eddy viscosity, which assumes that the principal axes of the turbulent stress tensor are

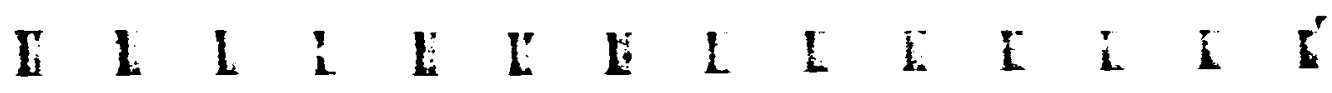


aligned with those of the mean strain-rate tensor. This is not true in shear flows, but the model coefficients are adjusted to give the right shear stress, which then makes the normal stresses incorrect. In thin shear layers where the normal stresses are not very important, good results can be obtained. But in rapidly strained flows, including flows at separation and reattachment, this model breaks down. The models do very poorly in 3-D (in the mean) flow, for example in the boundary layer at a wing-body junction or in the boundary layer on a swept wing.

The usual algebraic structure model is such that most predict an unrealizable turbulence state (e.g., impossible stresses and negative energy) during a sudden increase in the mean strain rate, for example, as a boundary layer approaches a 3-D obstacle. This contributes to the poor performance of these models under rapidly changing conditions.

Similar problems arise in heat transfer modeling, where it is usually assumed that the turbulent heat transport vector aligns with the mean temperature gradient. This is not true in shear flows, which exhibit anisotropic diffusion in which mean temperature gradients in one direction produce turbulent heat transports in other directions. Current models do not incorporate this important effect.

Scale evolution models that make use of more appropriate algebraic equations for the stress tensor have also been developed (the k-epsilon "algebraic" models). These can be constructed in a way that removes the unrealizablity described above, with the turbulent heat transport in the proper relationship to the mean temperature gradient. Models of this type are at a level of complexity near the maximum commensurate with use in large CFD codes for design calculations, and thus these methods would appear to deserve much more attention.

Practically all modeling of this type is limited to incompressible flows, and there is very little activity in extending these models to supersonic and hypersonic flows. Most of the original work and current development of these new models is taking place in Europe. There is some activity in extending these methods in the NASA laboratories, notably the NASA Ames Research Center, but very little other effort in the United States along these lines. It is apparent that significant improvements in the capabilities of these models can be achieved through use of data from direct

$\begin{array}{llllllllllllll}\text { I } & \text { I } & \text { L } & \text { I } & \text { L } & \text { L } & \text { E } & \text { L } & \text { E } & \text { I } & \text { E } & \text { I } & \text { I } & \text { E }\end{array}$ 
turbulence simulations and insight from experiments. The engineering importance of these models is such that the U.S. would benefit from a much more vigorous program in this direction.

\section{Transport Evolution Models}

These models are based on exact equations for the evolution of the transport terms in the TANS equations. The equations for the transport terms are derived from the parent Navier-Stokes equations, and are added to the TANS equation set. In addition, one must include at least one equation for a parameter carrying the turbulence length scale information; the dissipation-rate (epsilon) equation is commonly used. Local algebraic models are made for the higher (still one-point) statistical quantities in these equations. The "Reynolds stress transport" models, in which the Reynolds stresses become dependent variables of the partial differential equation system, are of this type. The survey revealed that only a few codes can incorporate such models, which add considerably to the cost of computation.

Most modelers regard the epsilon equation as providing length scale information, and some have criticized this modeling because it does not take different length scales in different directions into account. But in fact the epsilon equation provides Lagrangian timescale information, which can be thought of as information on the lifetime of coherent structures; the resulting length scales acting in transport terms can be shown to be different in different directions. This connection of the epsilon equation with coherent structure lifetime may explain why models based on the epsilon equation do surprisingly well.

Transport evolution models take explicit account of the history of both the scales and structure of the turbulence. These models allow the principal axes of the turbulent stress tensor to align differently from those of the strain-rate tensor. However, they require models for many other turbulence quantities, and the evolution is often determined by a delicate balance of these modeled terms, few of which have ever been measured. The net effect is that, while transport evolution models are fundamentally on stronger ground than lower-level models, they can perform poorly if they have not been finely tuned for flows very much like the one being studied. They are especially vulnerable if they have not

I $\quad$ L L L I $\quad$ E 
been developed on sound mathematical grounds, which has often been the case.

The problems of realizability that plague scale transport models also arise in transport evolution models, but in much more sophisticated ways. If the algebraic models for the higher-order terms are not properly configured, the model will respond incorrectly to rapid changes in the mean strain-rate and will predict unrealizable turbulence states. It is possible to construct these models properly so that this does not happen, but fully realizable models of this type are extremely complicated, involving a great number of tensor combinations of the dependent variables with many coefficients. Nevertheless, this complexity has been shown to be useful in providing much better models for mixing, buoyancy, and combustion. Hence, this type of modeling is beginning to find its niche. It is important to develop proper models of this type in order to assess their limits, to aid in the hierarchical development of simpler models, and for use in complex flow regions where their special capabilities are required.

Among the important effects for which this type of proper transport evolution modeling is likely to be useful are density gradients, rotation, high freestream turbulence, energy release, and other situations where the turbulence is strongly altered by some imposed effect. Engine flows form a prime example of such conditions. For example, turbulent reattachment, turbulent heat transfer, and stage losses easily can experience errors on order of a factor of two. Turbulent reattachment is controlled by turbulent transport of momentum normal to the separation bubble boundary; this transport is often strongly influenced by flow curvature in the streamwise direction, as well as by 3-D effects.

The ability to put these effects in parametric form in existing models is inadequate or nonexistent. Turbulent heat transfer, both external and internal to the blades, is strongly influenced by rotation and by pseudo-buoyancy associated with temperature fluctuations; the ability to develop parameters of these effects is inadequate or lacking for existing models. Stage losses are associated with secondary flows, as well as with separation and boundarylayer development. Development of parameters for turbulence in all of these cases in rotating systems is inadequate or absent for present models. Without doubt, properly developed transport evolution models can be made to handle these effects better, and such model development needs competent attention.

I I L I L I 
Similar needs arise in flows on airframes. Reattachment in such flows is nearly always 3-D, and the location of the reattachment line is determined by turbulent momentum exchange between the separation zone and the surrounding flow. This can involve complicated secondary flows and complex curvature-induced vortices. Existing models show the parameters of these effects poorly, if at all. The physics of the situation is relatively clear; what is needed is to apply the understanding of the physics to sophisticated model development.

There is hope that improved turbulence models can be used to elucidate these effects. Fundamental experiments needed to calibrate models for these effects are needed. The domain of both the models and the experiments need to be extended, especially to transonic and supersonic conditions.

Simplified transport evolution models, constructed in an ad hoc way so that they behave properly in the limits of strong strain-rate and guided by the more rigorous modeling theory, offer a possible compromise between the desire for rigor and the need for computational simplicity. Such models are probably the most complex that are likely to be used extensively in large CFD applications during the remainder of this century. Only one or two groups in the United States are active in this area.

\section{Probability Density Function Models}

Driven by problems in combustion modeling, there is increasing interest in turbulence models based on probability density functions (PDF). Most of the work has dealt with the joint PDF for the velocity components, density, and species concentrations at a single space-time point. The equation for the PDF is derived from the Navier-Stokes equations.

This restricted PDF is not sufficient to describe all statistics of a turbulence field (the infinite multipoint joint PDF would be required), but it does appear to offer some advantages. Specifically, the transport terms in the TANS equations do not need to be modeled because they are one-point statistical quantities that can be calculated from the PDF. However, other terms do need to be modeled.

The models cannot be based on data because none exist. Attempts to generate data from simulations on such flows are just now beginning. Because of the vast data required for model

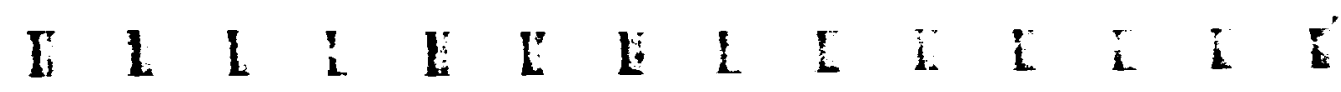

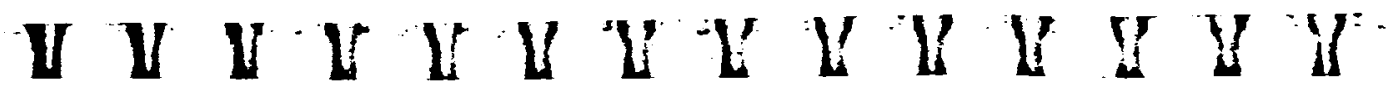


calibration, full simulations will very likely be the only way to calibrate PDF models.

The partial differential equation for the PDF needs to be solved in a high-dimensional space defined by the local fluctuation variables and the flow geometry. Hence, Monte Carlo computational methods are usually employed. Better numerical methods for solving partial differential equations in spaces of high dimension could accelerate the pace of PDF model developments.

In time these models may develop to the point where they are useful for studying isolated engineering problems in combustion. They are likely to be very useful in developing simpler computational models for such flows. It is not expected that they will be needed or used for flows not involving chemical reaction.

\section{Spectral Interaction Models}

The models described above are all one-point models in that the statistical quantities involved are correlations of turbulence quantities at a single point. Models that make use of two-point statistical quantities, such as the two-point velocity correlation, have also been developed. An equivalent representation is provided by the spectrum function, which can be calculated from the two-point correlation. Equations for the two-point correlation, or alternatively for the spectrum, can be derived from the NavierStokes equations. The resulting equations contain terms that must be modeled. The Direct Interaction Approximation is a model (in spectral space) of this type.

In principle these models would overcome a serious shortcoming of one-point models, namely their inability to deal with turbulence in which spectral nonequilibrium is important (large eddies out of equilibrium with the small eddies). This phenomenon occurs under rapid deformation, for example in the flow near a stagnation point or with sudden energy release. In practice this is not so easy because the two-point equations must be solved in a high-dimensional space defined by the coordinates of the two points. As a result of this complexity the development of these models has concentrated on homogeneous flows, where only the separation coordinates are important, and there have as yet been no engineering applications of the models.

The atmospheric sciences community in the United States, centered at the National Center for Atmospheric Research

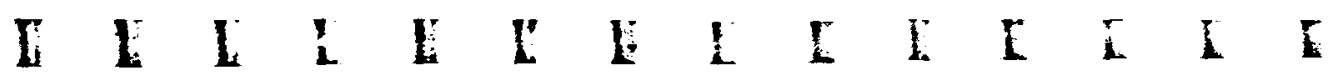


(NCAR), has led in the development of spectral methods, which have provided insight into turbulence physics but little in the way of computational tools for engineering. However, the French are actively attempting to bring two-point models to bear on aircraft problems, chiefiy through a cooperative effort between the university group at Lyon and the aerospace company, Dassault. A complete U.S. program on turbulence modeling certainly should include a small effort along these lines.

\section{Turbulence Simulations}

Direct numerical simulations of all important scales of turbulence (also called full turbulence simulations or FTS) are possible only at relatively low Reynolds numbers. Current computers can treat only the simplest flows in the vicinity of transition Reynolds numbers; by the end of this century the range will be extended upward by something less than an order of magnitude, still far below the range of most engineering applications. Nevertheless, these simulations provide extremely valuable data for use in developing simpler models of turbulence and transition.

Large eddy simulations (LES) use time-dependent equations averaged over the unresolved scales of motion, with models for the subgrid stress terms introduced by the averaging, to calculate the evolution of the large-scale field. The idea is that the smaller scales of turbulence can be modeled more simply since they adjust more rapidly to local conditions. The large, computed scales are responsible for most of the turbulent transport. Moreover, since the dominant eddies are resolved directly, a relatively unsophisticated turbulence model can be used for the small-scale turbulence.

It has been established that relatively simple subgrid models allow accurate LES of simple turbulent flows (e.g., channel flow) where no-slip or periodic boundary conditions can be applied. To become useful in engineering calculations, better methods for modeling the unresolved turbulence, particularly near walls, are needed, and methods for creating turbulent inflow conditions with the proper structure are needed. Subgrid turbulence models need to be extended to variable resolution grids and to be made dynamic so that the small scales can be out of equilibrium with the large scales. Active research on all of these problems is under way, primarily for incompressible flow. These programs need to be extended to flows with large separated regions and to supersonic

$\begin{array}{llllllllllllll}\text { I } & \text { I } & \text { L } & \text { L } & \text { E } & \text { I } & \text { E } & \text { L } & \text { E } & \text { I } & \text { E } & \text { i } & \text { I } & \text { I }\end{array}$ 
flows. This expansion in effort will require considerably more manpower than is presently deployed in LES development.

Perhaps early in the next century LES will begin to become a useful tool for design computations. LES will be essential for such problems as sound generation by, and transmission through, boundary layers. But for the next decade or so the first use of LES will be to provide data that cannot be measured experimentally on simple flows to guide the development of simpler models of the scale evolution and transport evolution type.

The first engineering problems likely to be tackled by LES are those in turbomachinery. Here the freestream turbulence levels are very high, the scales are very large, the flows are far from equilibrium and often contain embedded vortices, and it may prove very difficult to develop a generalized turbulence model to handle this complexity. Moreover, the Reynolds numbers in these flows are relatively low, and so the flows are closer to what can be reached by FTS. Indeed, FTS and LES may prove to be the only viable means for computing such flows. With a strong, ordered program of development, it should be possible to begin LES and FTS exploration of these flows in the next decade.

The United States is clearly the world leader in developing LES and FTS, which are being pursued vigorously in NASA centers and other government laboratories and in a few key universities with supercomputer access. However, the Europeans and Japanese are very actively exploring LES for industrial applications, and the French Electricite de France (EDF) is probably the world leader in industrial development of LES. The U.S. lead in this area is threatened by these developments, especially in Japan where Cray performance is available on the national network for under $\$ 100$ per hour.

With the advent of a set of national supercomputer centers, formed by the National Science Foundation (NSF), an expansion of effort in LES and FTS is beginning. A typical doctoral dissertation project involving a fundamental FTS or LES may involve several hundred hours of supercomputer computation. In view of this enormous expense, it is important that such computations be very well planned, not unknowingly duplicated at another center, and that the computed data be saved and archived so that it can be used by other investigators. At present there is no national coordination of these efforts and few serious attempts at archiving, thus many of these very heavy computations will be of dubious and

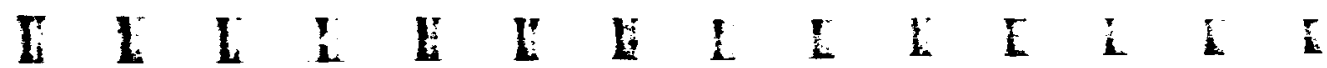


temporary value. Some method for coordination of these activities is needed.

\section{Emerging Ideas}

There are a number of emerging ideas that may have an impact on turbulence modeling and simulation. The concept of fractal geometry, in which structures are seen as containing smaller structures of similar shape, which in turn contain smaller structures of similar shape, and so on, is an appealing model for the smaller scales of turbulence. It is possible that this concept, and the related quantifications of renormalization theory, will lead to formulation of better subgrid scale models for LES, and perhaps to improved algorithms for LES. These ideas deserve attention, but are at present far from implementation in practical problems.

Dynamical systems theory, which deals with discrete coupled systems, shows that systems which appear to be deterministic can exhibit chaos, which is reflected in a broad spectral response that appears to be random. Such a system is said to possess a "strange attractor." In essence, the nonlinear coupling reduces the number of parameters (dimensions) required to describe states of the system. The statistics of a low-dimensional system could presumably be described by the coupled dynamics of a very few modes, and so the hope is that very complex systems might thereby be described more simply. There is some indication that certain turbulent flows may be of this type. However, if the dimension is very high, as appears to be the case in shear flows, statistical approaches and LES would probably remain the preferred alternatives.

These ideas may find their way into LES in a more immediate way. Ideas for using the dynamical system equations to generate chaos, either for the subgrid model or for turbulent inflow conditions, have been suggested. Initial explorations of these ideas are under way.

An old type of stochastic model, the Cellular Automata (CA) model, is being reexplored and was recently publicized by Los Alamos as revolutionizing CFD. In this approach a very simple bit switching algorithm is executed; the statistics of the resulting bit states have been shown, in the limit of many elements and switches, to approach the solution of partial differential equations resembling the Navier-Stokes equations. The advance at Los Alamos was in the algorithm used for the bit switching, which

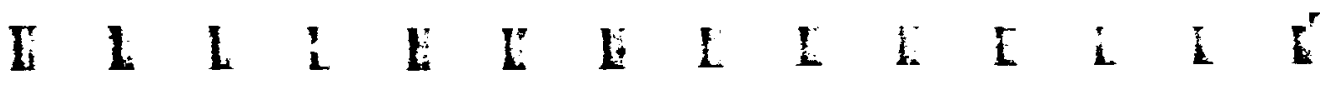


makes the process run faster. However, it has only been shown for 2-D cases, and really has yet to produce anything resembling true turbulence. Examinations of this work have shown that the advances in the CA algorithm are certainly significant for CA theory, but that the prospect for a serious impact on modeling practical turbulent flows, at high Reynolds numbers and especially at high Mach numbers, is remote. For equivalent precision, the cost of CA models is prohibitive.

The existence of large-scale structures in turbulent shear flows has been recognized for two decades. But this obviously important physical information has as yet to find its way into turbulence models, except through the interpretation of the time-scale information carried by the epsilon equation as a large-eddy coherence time. A viable turbulence model based on the concept of coherent structures would seem a logical target for a concentrated effort in turbulence modeling, but no such model has been put forth as yet. This would seem to be an avenue worthy of exploration. Although this has occurred to many capable researchers, the right combination of ideas has yet to emerge.

A method for turbulence simulation that does recognize coherent structures is vortex dynamics, in which one follows the motion of concentrated vortices in an inviscid flow. These methods are reasonably well-developed for incompressible, 2-D, inviscidly dominated turbulent flows. Some special 3-D incompressible flows dominated by concentrated vortex structures have also been treated. Extensions to compressible flow and to vorticity diffusion may not be possible, and so this method of turbulence simulation may be quite limited in practical problems. Nevertheless, it has provided and will continue to provide valuable insight into the physics of large-scale turbulent motions that undoubtedly will be helpful in structuring simpler turbulence models. Further development of this technology should be an important component of future turbulence research.

\section{CURRENT STATUS OF TURBULENCE MODELING}

The discussion in the previous section highlighted the problems and potentials of turbulence modeling. Table 6 presents an evaluation of the state of turbulence modeling as it applies to

I 1 L L I 
TABLE 6 Status of Turbulence Modeling

\begin{tabular}{|l|c|c|c|c|}
\hline \multirow{2}{*}{ Flow } & \multicolumn{4}{|c|}{ Model } \\
\cline { 2 - 5 } & $\begin{array}{l}\text { Local } \\
\text { Algebraic }\end{array}$ & $\begin{array}{l}\text { Scale } \\
\text { Evolution }\end{array}$ & $\begin{array}{l}\text { Transport } \\
\text { Evolution }\end{array}$ & $\begin{array}{l}\text { Large Eddy } \\
\text { Simulation }\end{array}$ \\
\hline Simple Shear Flow & 2.0 & 2.0 & 3.0 & 3.0 \\
Flow Complexity: & & & & \\
Scalar Transport & 1.0 & 2.0 & 3.0 & 3.0 \\
Separation/Reattachment & 1.0 & 1.0 & 2.5 & 0.0 \\
Rotation/Curvature & 1.0 & 1.5 & 2.5 & 3.0 \\
High Strain Rate & 1.0 & 1.5 & 0.0 & 3.0 \\
3-D Mean Flow & 1.0 & 1.5 & 0.0 & 0.0 \\
Supersonic Flow & 1.0 & 1.0 & 0.0 & 0.0 \\
\hline Scale: & & & \\
0 Not tried yet & & & \\
1 Tried with generally poor results & & \\
2 Demonstrated with fair success \\
3 Case has been made that it is useful \\
4 Being used productively in research \\
5 Operationally effective in codes requiring its capabilities \\
\hline
\end{tabular}

simple shear flows and flow features characteristic of complex engineering flows. The four main types of turbulence models and simulations viewed as having significant impact on CFD in the remainder of this century are rated in terms of their states of development and promise. This subjective evaluation is based on performance at the 1981 Stanford Conference on Computation of Complex Turbulent Flows, recent evaluations by NASA personnel, input gathered from various members of the committee, and the working knowledge of those committee members most active in turbulence modeling and simulation.

\section{FINDINGS}

Turbulence modeling is becoming a pacing item in the development of CFD codes for many engineering design applications. The modeling problem is acute with regard to aircraft engine design codes, and is becoming a key factor in airframe codes in situations where viscous effects dominate. In many of today's codes the significance of turbulence modeling is masked by excessive numerical diffusion in the algorithms. Once this numerical diffusion is eliminated the significance of the turbulence model will become

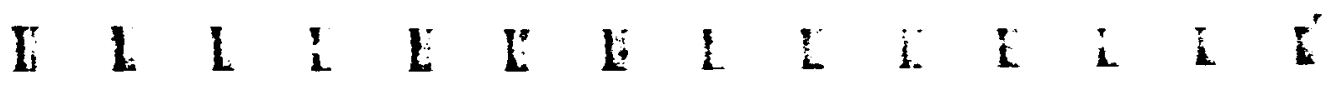


clearly evident, It is anticipated that turbulence modeling will be an essential element in CFD codes for the remoinder of the century.

There is no substitute for modeling of some sort. LES or FTS may help develop models and even be used in some critical design situations, but they will not serve most of the needs in design codes in the foreseeable future. Furthermore, better turbulence modeling is also needed for LES.

The committee's level of effort survey (see Chapter 3 and Appendix C) indicated that only about 4 percent of the effort in CFD development is being devoted to the improvement of turbulence models, and only about 5 percent to turbulence simulations. Thus, less than 10 percent of the CFD effort is being applied to developing better methods for treating what is possibly the greatest challenge for applied CFD. It is believed that this effort is low in view of the importance of the problem.

\section{RECOMMENDATIONS}

Turbulence model improvements are possible and will be made most rapidly by careful interaction among direct simulation, large eddy simulation, theory, model development, and experimentation. It is essential that this development be carried out in cooperation with the user community and tailored to the needs of CFD code developers. The effort should be conceived initially in those few places where a critical mass of talent can be achieved, but also must extend as broadly as possible so that a wide diversity of competent ideas are brought to bear.

The importance of turbulence and transition in engineering systems needs to be reemphasized. Perhaps the exciting new developments in the ability to control turbulence, through understanding of its physics, can be used to spark the needed reawakening. Special programs of the type that have given CFD and supercomputers such a big push in the last decade should be considered as a means for accelerating the pace of developments in turbulence modeling and simulation. A renewed interest in fundamental graduate education in turbulence will be necessary to produce the proper training required for this task.

Supercomputer-based research employing full turbulence simulations and large eddy simulations needs to be continued and coordinated to eliminate unnecessary duplication of effort and to ensure that data are archived in a way that maintains them for future

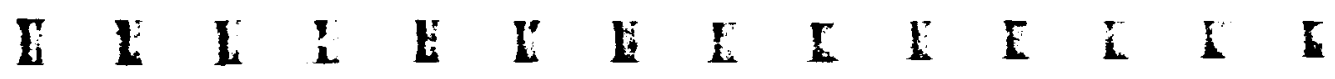

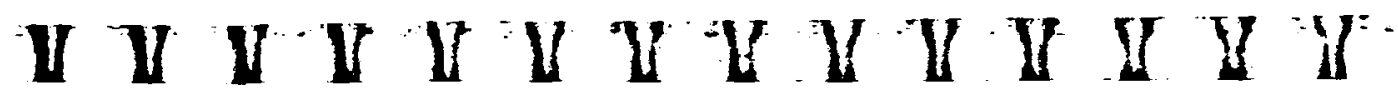


analysis. These simulations form a critical basis for fundamental understanding and a framework for development of practical turbulence models.

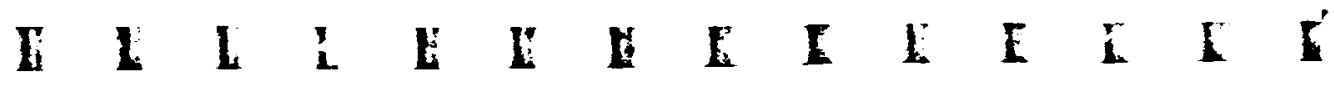
I I I I I V I V I I I I I I I 


\section{APPENDIXES}

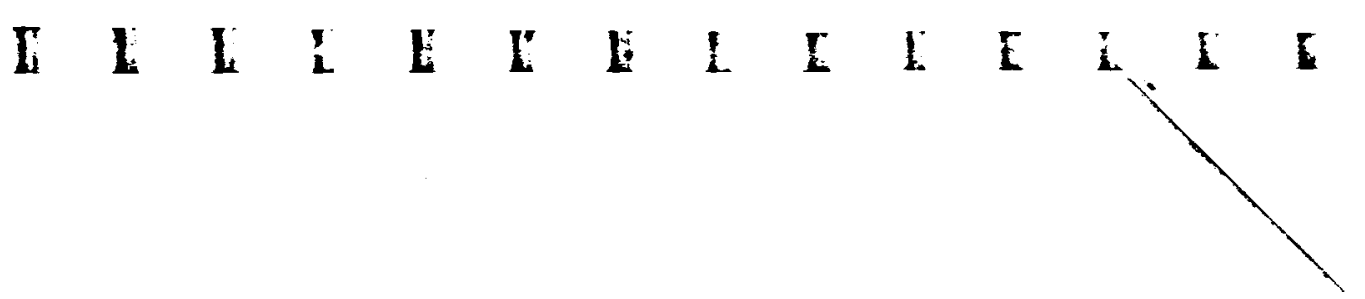

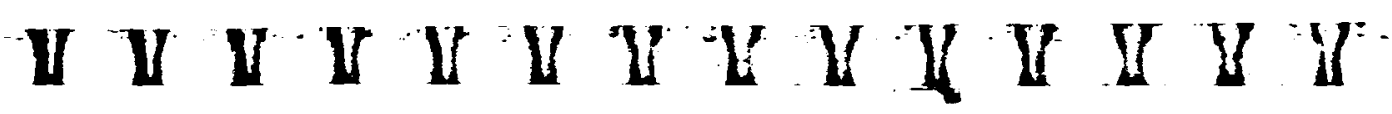




\section{Appendix A \\ Trends in Computer Hardware \\ Affecting CFD}

Computational fluid dynamics (CFD) will evolve in response to changing computational, graphical, and diagnostic capabilities as well as new models, algorithms, and eventual applications. The anticipated future environment in which CFD will be pursued will influence significantly the algorithms that are used and the methodology by which computational research is pursued. Detailed projections concerning these emerging capabilities and approaches are bound to be highly uncertain, as with most emerging new technologies. Detailed consideration of future hardware trends is beyond the charge of the committee, nevertheless, certain future trends are evident.

As the cost of fast computer memory decreases, the appearance of memory-intensive models using many millions of words of primary data is already beginning to influence algorithm selection in favor of faster, more efficient algorithms that make less use of each computational degree of freedom. This means that much more data will have to be analyzed and stored in the future. This trend is likely to accelerate because a gradual shift to highly parallel computing seems inevitable, and laser disk storage is becoming a reality. Fine-grained parallelism, through pipelining and vector processing, has taken over the supercomputing scene in the last 15 years. It seems likely, given the intrinsic limitations imposed by the speed of light and component technology, that this trend toward increasingly extensive parallelism will continue in the future. Already systems with tens of thousands of small processors are being produced and sold commercially, and the U.S.-manufactured supercomputers are multiprocessors.

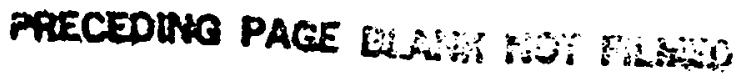

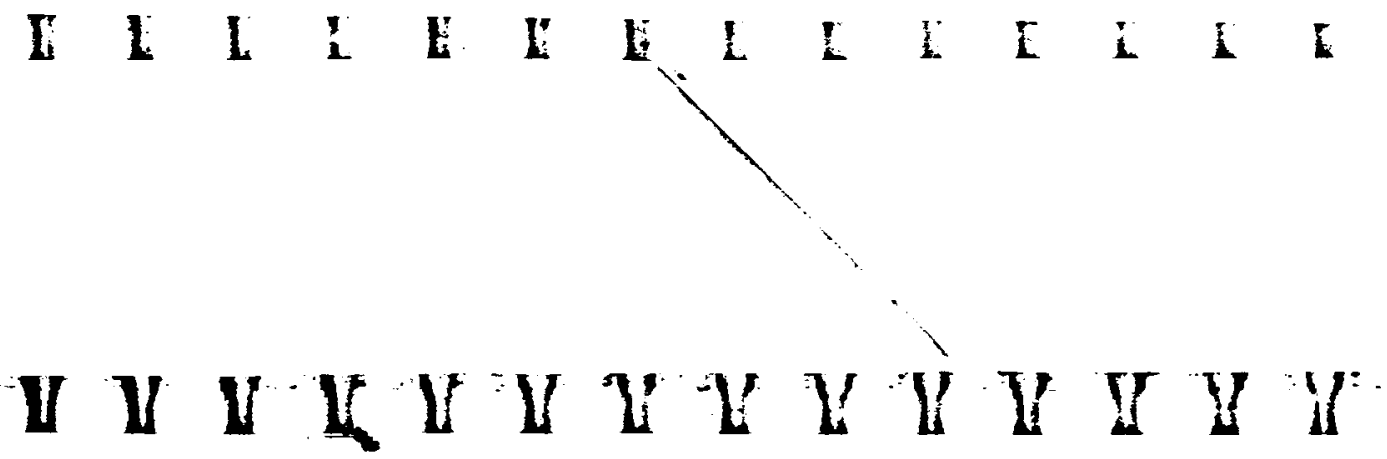


Using this brand of multitasking, i.e., coarse-grained parallelism, is very different from scalar or vector processing. Different algorithms will appear to be most favorable for this kind of supercomputing, and new programming methodologies will have to be adopted. In some cases, simpler, less-accurate solution algorithms and grids will be adopted because they lend themselves better to computation on highly parallel processors. In other cases the mathematical models themselves will evolve to reflect the computer systems on which they are being implemented. Multilevel parallelism, for example a vector of loosely coupled array processors or a large closely coupled matrix of small scalar processors, will force additional changes in modus operandi. A number of fairly high-performance systems are available commercially at modest price so the CFD community should have a reasonable corporate understanding of these capabilities when the time comes to replace Crays and Cyber 205s.

Continually improving supercomputer performance has already brought another evolutionary change that may force the CFD community to exhibit a broader mix of skills than has been necessary to date. The feasibility of large 3-D calculations on a daily (and even hourly) basis deluges the researcher in data which he may be ill-equipped to handle. The numbers are formidable in analyzing multidimensional evolving simulations. Special diagnostic and data base management software are needed. Simply storing restart dumps from a three-dimensional (3-D) calculation (e.g., 40 megabytes per dump) at frequent enough intervals to reconstruct some of the important temporal variations (e.g., every 250 cycles for 25,000 steps) fills several large disks.

Interactive graphics methods and the associated hardware and software will become absolutely crucial to monitoring, interpreting, understanding, and presenting the computational results. Although these are not new issues, they are becoming dominant considerations for the future. High-performance graphics workstations will not be sufficient to solve the problems. It is also necessary to move data from the computational and bulk storage facilities directly onto the graphics screen at multimegabyte/sec speeds. Reasonably continuous variation ( $\geq 12 \mathrm{frames} / \mathrm{sec}$ ) and reasonably high resolution $(1,024 \times 1,024$ pixels, for example) require average data transmission at the $12 \mathrm{Mbyte} / \mathrm{sec}$ level. For comparison, MC 68000-based graphics workstations can accept new data at only $150-200 \mathrm{Kbytes} / \mathrm{sec}$ today and thus treat only

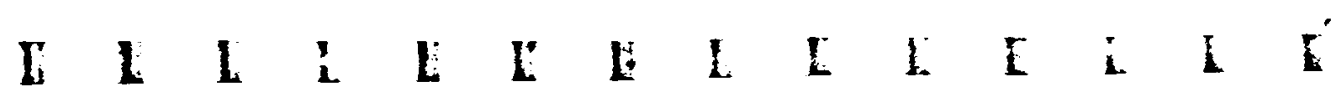

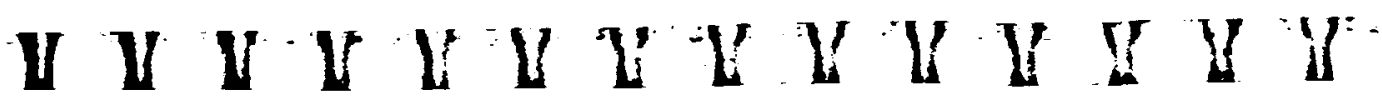


one image at a time. Electronic storage of 1000-frame "movie" sequences for analysis, about 1-2 minutes when projected, will require about 1 gigabyte of storage. The CFD community will have to pursue this technology base steadily in using the emerging parallel processing systems effectively. A $256 \times 256$ interactive graphics system is required simply to monitor the instantaneous performance of a $64 \mathrm{~K}$ processor system with only 1 pixel of color per processor.

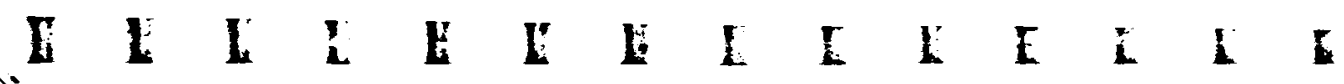

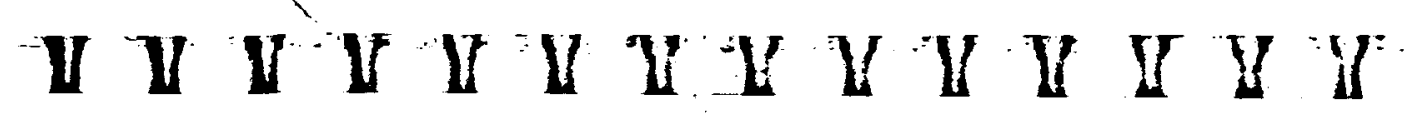




\section{Appendix B}

\section{NATIONAL RESEARCH COUNCIL}

COMMISSION ON ENGINEERINC AND TECHNICAL SYSTEMS

2101 Constitution avenue Washington. D C 20418

AROMAUTCS AND SPACE

July 24, 1985

Dear Colleague:

The National Aeromatics and Space Administration has requested the Aeronatics and Space Engineering Bcard of the National Research Council to assess the current status of computational fluid dynamics (CFD) in the design of aerospace systens and to make recommendations regarding future needs. We would appreciate your assistance in helping to determine the state of the art in computational codes for aerodynamic and aerothermodynanic applications.

Enclosed is a questionnaice designed to obta in information about CFD codes currently in use. We are seeking a broad, representative sampling of codes and have tried to design the questionnaire to require a minimum of your time to respond and yet to provide useful information for the purposes of the survey. If you, or those on your staff who are developing or using CPD codes, would return completed forms for individual codes by the middle of August, it would help us proceed to the next phase of our study. I believe you will find the questionnaire self-explanatory, but I would like to say that any other comments in addition to the information sought will be welcome. If you have any questions, please telephone me at 817/763-1609 or the study Director, Dr. Robert H. Korkegi, at 202/334-2855.

For your information a list of study participants is enclosed with the questionnaice. We will, of course, provide you with copies of the report when we have completed our task.

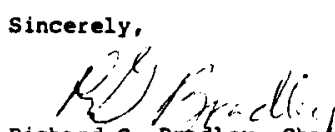

Richard G. Bxadley, Chadrman

Assessment of Current fnd ruture Capabilities

in Computational Fly/id Dynamics

Enclosures: 2 copies of questionnaire

study nember ship list

stamped return envelope

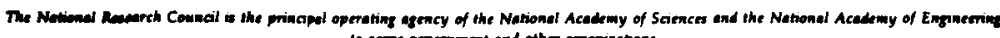

to ereve goovernment and other organizabons

86

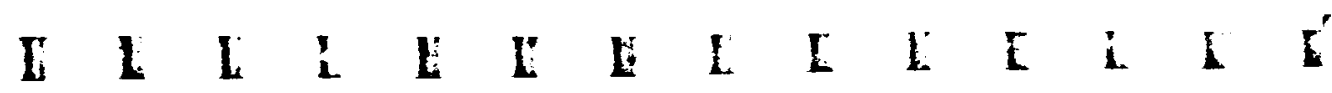

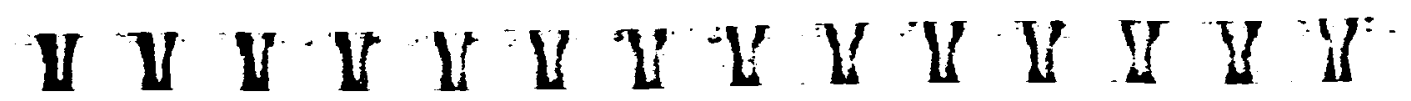


CFD CAPABIIITY ASSESSMENT

I. Category of Application (circle appropriate itens)

A. Flow Type
1. External Air Vehicle
2. Propulsion System
3. Hypersonic Flow
+. Other

B. Geometry Applicability

1. External Air Vehicle

a. Airfoil, aiffoil flap

b. wing, wing-body, wing body-nacelle/stores,

c. Complex configurations - multi surface flaps,

propulsion interactions, etc.

d. Other

2. Propulsion System
Inlet, nozzle, nacelle, duct, mixer
b. Fan, compressor, turbine
c. Combustor, afterburner, plume
d. Propeller, rotor
a. Rocket components
f. Other

3. Hypersonic Flow
a. Body, wing
b. Wing body, wing body $f$ in
c. Integrated propulsion system, plume, etc.
d. Other

C. Range of Applicability (shade applicable range)

Mach:
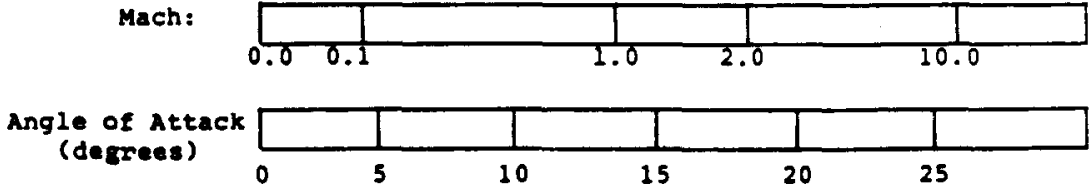

Separated Flow

YES $\square$ NO

Energy Maltion (propulaion)

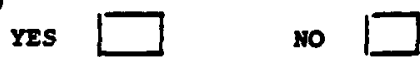

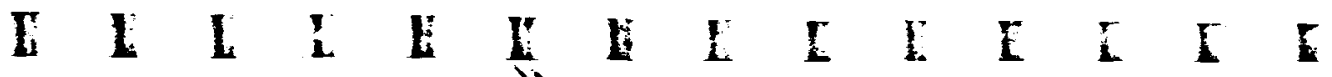

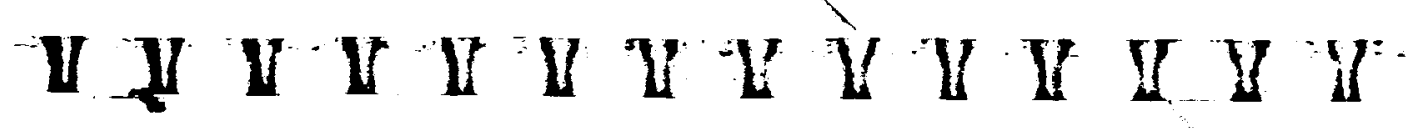


CTD CAPABILITY ASSESSMENT

Page 2

II. Equations Solved (Check Appropriate Element)

A. Equations

CONTINUTH

Inviseid

Linear

Small Disturbance

Full Potential

Euler

other

Vilecous

Laminar Boundary

Layer

Turbulent Boundary

Layer

Full Laminar Flow

Perabolized N.S.

Thin Layer N.S.

Full Navier Stokea

Other

PARTICUIATE

Monte Carlo

other

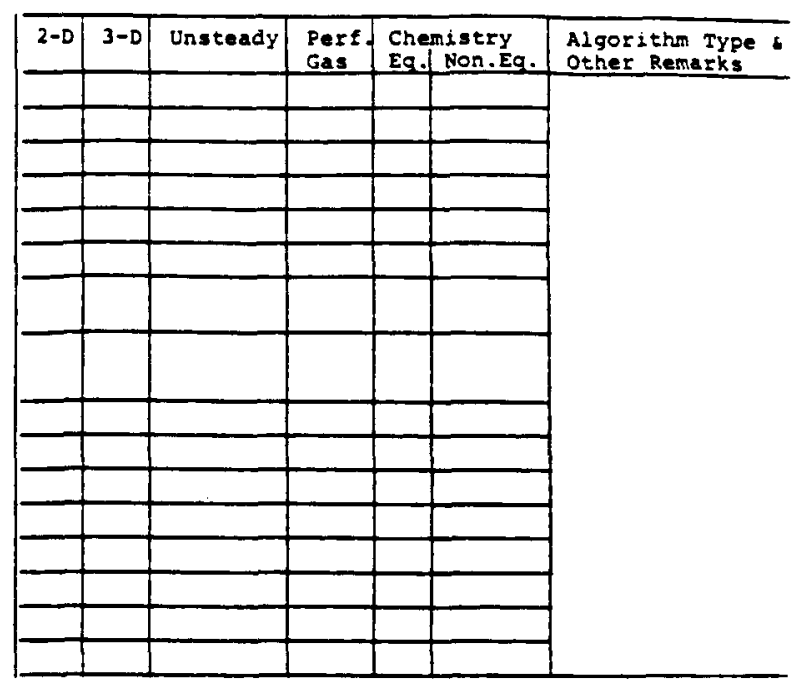

B. Turbulence

For viscous solutions give name of turbulence model used if poseible

Otherwlse indicate turbulence model used as follow:

sddy transport coefficient: (mixing length model)

k-e with eddy viscosity model

k-c with other algabraic atress models

Reynolde etress traneport equation

other

c. Mdditional reatures

Electromagnetic

Combustion

other

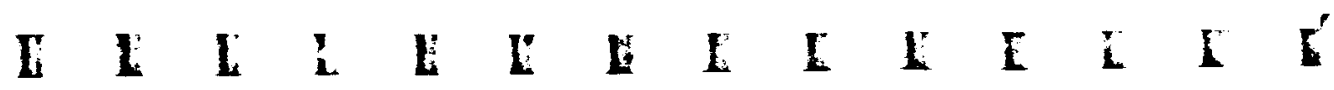


CTO CAPABILITY ASSESSMENT

Page 3

III. Useability

A. Code identifier/rame

B. Position in development eycle (See Attachment A)

\begin{tabular}{c|c|c|c}
\hline II & III & IV & $v$ \\
$\begin{array}{c}\text { Demonstration } \\
\text { Evonfidence }\end{array}$ & $\begin{array}{c}\text { Evalving inteo } \\
\text { usable code }\end{array}$ & $\begin{array}{c}\text { Learning how to } \\
\text { use it effectively }\end{array}$ & $\begin{array}{c}\text { Mature cepability } \\
\text { (Design tool) }\end{array}$ \\
\end{tabular}

c. Type of user required

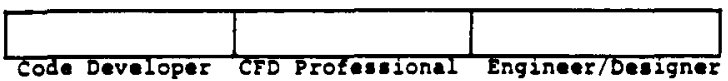

D. Computer type/resources required

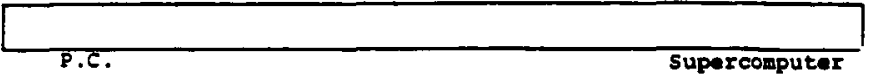

E. Set up requirenent:

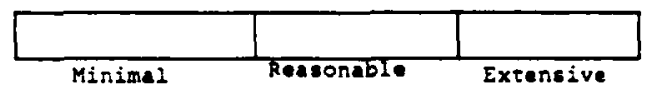

F. Typical teatures

CPU time

on Computer

Mmory requirement:

G. What are the anjor 1 imitations/restrictions?

8. What are the special capabilities?

I. What edditional work is needed?

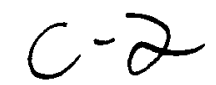

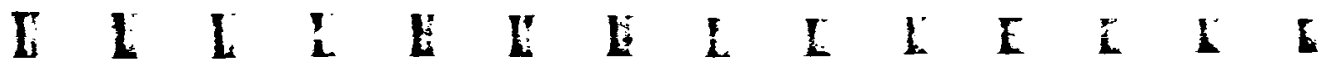

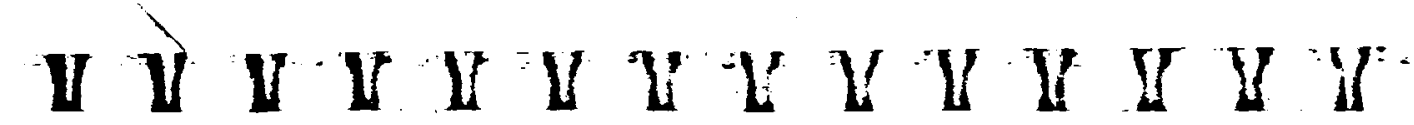


CFD CAPABIIITY ASSESSMENT

Page 4

IV. Believability

A. What is the confidence level of the solution's validity?

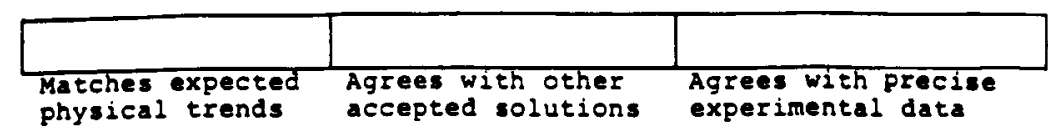

B. Has the sensitivity to grid size/geometry been established?

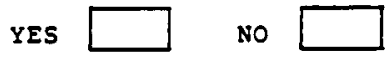

c. What flow quantities are output?

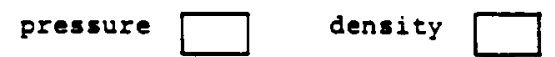

gas composition

temperature

velocity

What integrated quantities?

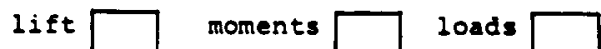

streamline tracing $\square$ heat transfer $\square$

drag: wave $\square$ profile $\square$ induced $\square$ friction $\square$

propulsion: component efficiency $\square$ pressure drop $\square$

cycle efficiency

D. For what cases has the code been validated?

Case Published? Where

1.

2 .

3.

Name affiliation:

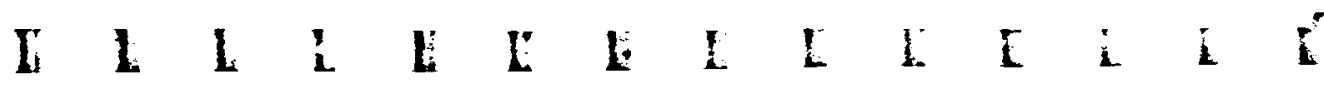



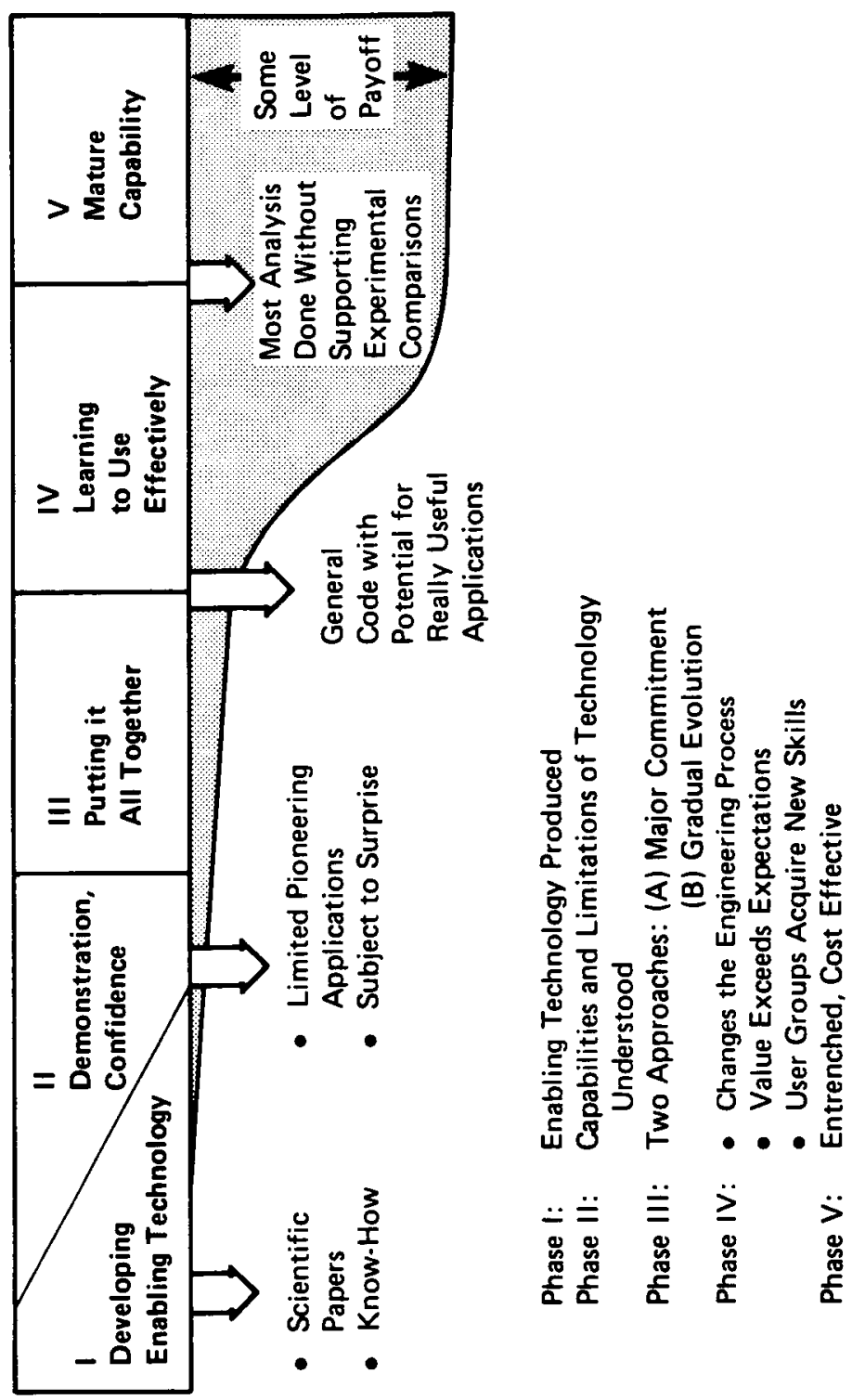

$\begin{array}{llllllllllllll}\text { I } & \text { I } & \text { L } & \text { I } & \text { E } & \text { I } & \text { E } & \text { L } & \text { I } & \text { L } & \text { I } & \text { I } & \text { I } & \text { I }\end{array}$

I บ V V I V U V V U I I V V IT 


\section{Appendix C}

NATIONAL RESEARCH COUNCIL

COMMISSION ON ENGINEERINC AND TECHNICAL SYSTEMS

210 Constitution Avenue hashington, D.C. 20418

AERONAUTICS AND SPACE

ENGINEREING DOARD

October 2, 1985

Dear Colleague:

As part of an assessment of current capabilities and future directions in computational fluid dynamics, we are making an assessment of the level of effort being devoted to fundamental developments in numerical methods--algorithms, grids, turbulence model development, code development, experiments, and code evaluation. We would very much appreciate your assistance in obtaining this information on the programs that you sponsor that involve these areas. On the back of the attached form you will find a glossary describing the information that we request on the form. In a few days a member of our comnittee will call you to answer any questions that you may have. We realize that the completion of this form will require subjective judgement on your part, and we very much appreciate the time you will take in making these judgements.

We would appreciate receiving the information, together with any comments you may care to provide on the trends of the research in your program, by November 1, 1985 . Please return the forms directly to this office.

Yours truly,

Robert H. Korkegi

Study Director, Assessment of

Current Capabilities and Future

Directions in Computational

Fluid Dynamics

Enclosures: 1. Survey with glossary

2. Committee membership list

The National Reseavech Council is the oprincipal oporating agency of the National Acedemy of Saiences ond the Netional Academy of Engineening

ro erve government and other orgenizenions

92

$\begin{array}{lllllllllllllll}\text { I } & \text { L } & \text { L } & \text { I } & \text { E } & \text { I } & \text { L } & \text { L } & \text { L } & \text { L } & \text { I } & \text { i } & \text { I } & \text { I }\end{array}$ 


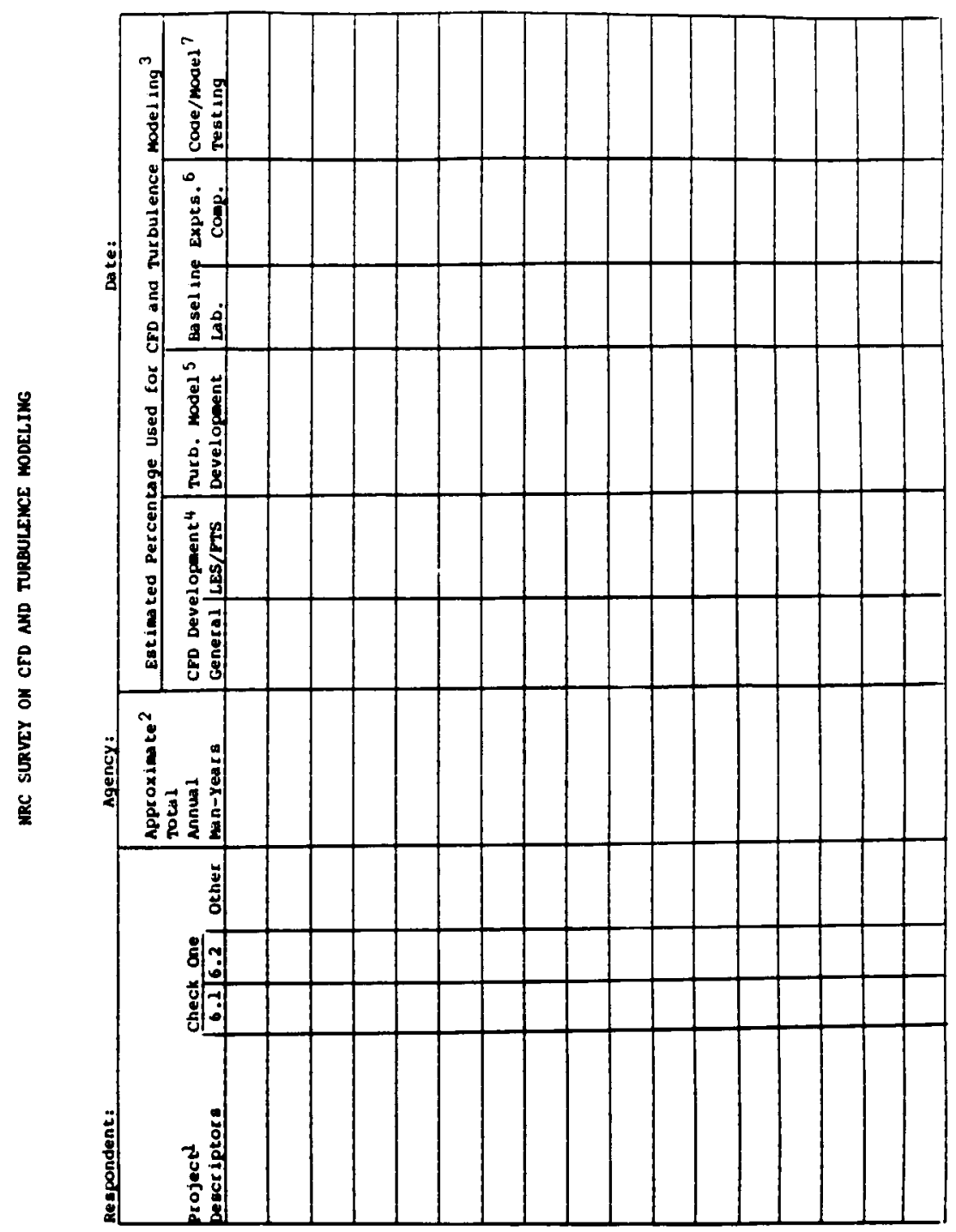

I I I I E I I I I I I I I I I I

I I I I I I V V V I Y I 


\section{Glossary}

1. Project Descriptors: essence of project in a few key words 6.1 $=$ basic research, $6.2=$ applied $\mathrm{R} \& \mathrm{D}$.

2. Approximate total annual man-years: estimated effort for the project; for university, research, you may wish to use 0.2 manyears per faculty member and 0.5 man-years per graduate student as a standard.

3. Estimated percentages used for CFD/Turbulence Modeling: $\%$ of listed total effort used in each category, based on your understanding of the project.

4. CFD Development: development of algorithms, grid generators, diagnostics or display software;

a) General capability for potential flow, Euler, and Navier-Stokes solvers

b) LES/FTS for time-dependent, three-dimensional eddy simulation

5. Turbulence Model Development: rigorization and significant generalization of existing models; new ideas for model formulation; distillation of simple models from complex ones; fabrication of special models for simple cases.

6. Baseline Experiments: canonical experiments designed to guide/ calibrate/evaluate models; complex experiments used to evaluate models and codes.

a) lab. laboratory experiments for above

b) comp. eddy simulation for above

7. Code/Model Testing: code execution for evaluation of codes and models.

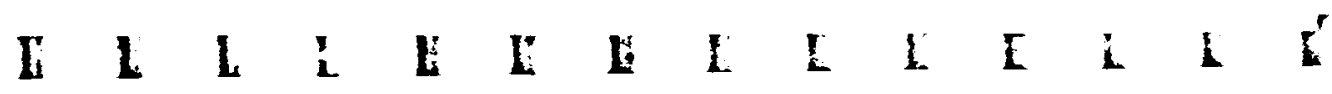

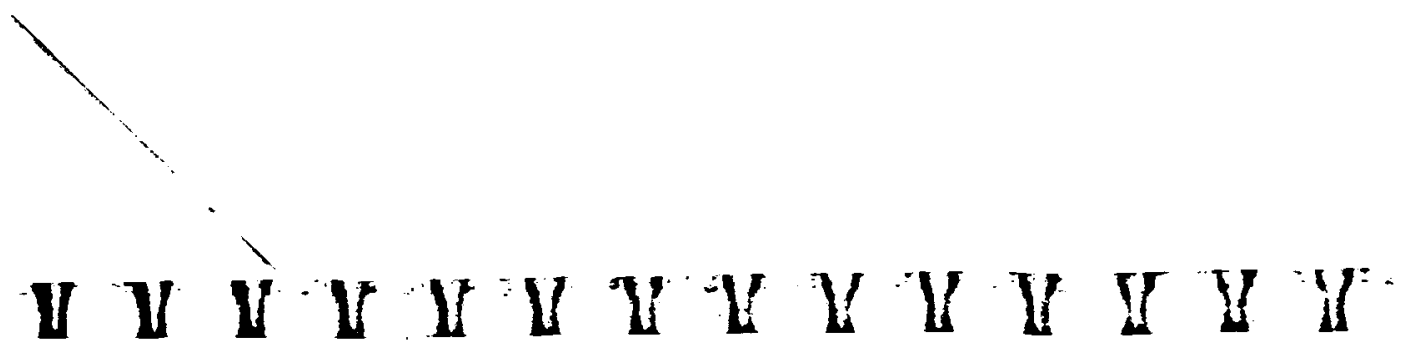




\section{Appendix D \\ List of Contributors to CFD Questionnaire}

Persons from the following organizations responded to the questionnaire in Appendix $B$ with data regarding specific CFD codes.

ACUREX CORPORATION

Mountain View, California

AEROSPACE CORPORATION

Los Angeles, California

AIR FORCE WEAPONS LABORATORY

Kirtland Air Force Base, New Mexico

AMTEC ENGINEERING, INC.

Bellevue, Washington

ARNOLD ENGINEERING DEVELOPMENT CENTER

Arnold Air Force Station, Tennessee

BOEING AEROSPACE COMPANY

Seattle, Washington

BOEING COMMERCIAL AIRPLANE COMPANY

Seattle, Washington

BOEING MILITARY AIRPLANE COMPANY

Seattle, Washington

CALSPAN FIELD SERVICES/AEDC

Arnold Air Force Station, Tennessee

COLORADO STATE UNIVERSITY

Fort Collins, Colorado

CORNELL UNIVERSITY

Ithaca, New York

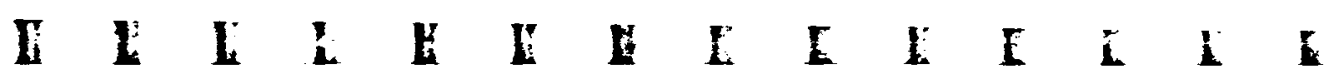


COLUMBIA UNIVERSITY

New York, New York

DOUGLAS AIRCRAFT COMPANY

Long Beach, California

DCW INDUSTRIES, INC.

La Canada, California ERA, INC.

Albuquerque, New Mexico

FLOW RESEARCH COMPANY

Kent, Washington

GENERAL DYNAMICS CORPORATION

Fort Worth, Texas

GENERAL ELECTRIC COMPANY

Cincinnati, Ohio

GENERAL MOTORS CORPORATION (Allison Gas Turbine

Operations) Indianapolis, Indiana

GRUMMAN AEROSPACE CORPORATION

Bethpage, New York

GRUMMAN CORPORATION

Bethpage, New York

HAMILTON STANDARD/UNITED TECHNOLOGIES

Windsor Locks, Connecticut

INSTITUTE FOR COMPUTATIONAL STUDIES

Fort Collins, Colorado

IOWA STATE UNIVERSITY

Ames, lowa

LYNDON B. JOHNSON SPACE CENTER

Houston, Texas

LOS ALAMOS NATIONAL LABORATORY

Los Alamos, New Mexico

GEORGE C. MARSHALL SPACE FLIGHT CENTER

Huntsville, Alabama

MASSACHUSETTS INSTITUTE OF TECHNOLOGY

Cambridge, Massachusetts

McDONNELL AIRCRAFT COMPANY

St. Louis, Missouri

McDONNELL DOUGLAS RESEARCH LABORATORY

St. Louis, Missouri

NASA AMES RESEARCH CENTER

Moffett Field, California

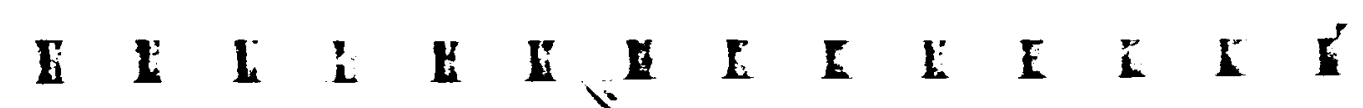


NASA JOHNSON SPACE CENTER

Houston, Texas

NASA LANGLEY RESEARCH CENTER

Hampton, Virginia

NASA LEWIS RESEARCH CENTER

Cleveland, Ohio

NAVAL SURFACE WEAPONS CENTER

Silver Spring, Maryland

NIELSEN ENGINEERING RESEARCH, INC.

Mountain View, California

NORTHROP CORPORATION

Palos Verdes Peninsula, California

OHIO STATE UNIVERSITY

Columbus, Ohio

PEDA CORPORATION

Palo Alto, California

PENNSYLVANIA STATE UNIVERSITY

University Park, Pennsylvania

PRATT \& WHITNEY (UNITED TECHNOLOGIES)

East Hartford, Connecticut

PRINCETON UNIVERSITY

Princeton, New Jersey

ROCKWELL INTERNATIONAL

Canoga Park, California

RUTGERS UNIVERSITY

Piscataway, New Jersey

SANDIA LABORATORIES

Albuquerque, New Mexico

SANDIA LABORATORIES

Livermore, California

SCIENCE APPLICATIONS INTERNATIONAL CORPORATION

Princeton, New Jersey

SCIENCE APPLICATIONS INTERNATIONAL CORPORATION

Wayne, Pennsylvania

SYSTEMS, SCIENCE AND SOFTWARE

San Diego, California

UNITED TECHNOLOGIES RESEARCH CENTER

East Hartford, Connecticut

UNIVERSITY OF ARIZONA

Tucson, Arizona

I $\quad$ I I I 
UNIVERSITY OF CALIFORNIA AT DAVIS

Davis, California

UNIVERSITY OF TENNESSEE

Kerrsville, Tennessee

UNIVERSITY OF TEXAS AT AUSTIN

Austin, Texas

VRA, INC.

Blacksburg, Virginia

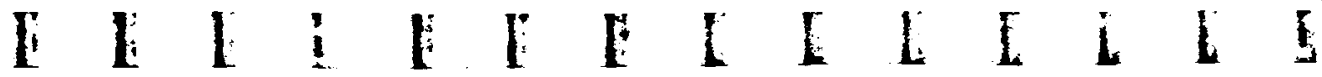

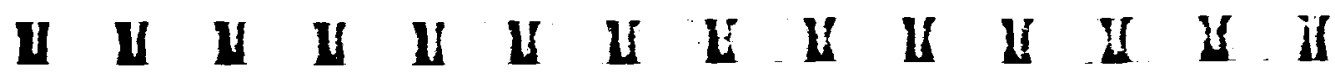

